\title{
Optimizing Budget Allocation in an Integrated Water and Sanitation System Using Optimal Control Theory
}

\begin{tabular}{c} 
A Dissertation \\
Presented to \\
the Faculty of the School of Engineering and Applied Science \\
University of Virginia \\
\hline
\end{tabular}

\author{
In Partial Fulfillment \\ of the requirements for the Degree of \\ Doctor of Philosophy \\ (Systems Engineering)
}

by

Rujira Chaysiri

August 2016 
(C) 2016 Rujira Chaysiri 


\section{Approval Sheet}

This Dissertation is submitted in partial fulfillment of the requirements for the degree

of

Doctor of Philosophy

(Systems Engineering)

Rujira Chaysiri

This Dissertation has been read and approved by the Examining Committee:

\begin{tabular}{c}
\hline Dr. Garrick E. Louis, Advisor \\
\hline Dr. Gerard Learmonth, Committee Chair \\
\hline Dr. William T. Scherer \\
\hline Dr. James H. Lambert
\end{tabular}

Dr. Zoran Grujic

Accepted for the School of Engineering and Applied Science:

Craig H. Benson, Dean, School of Engineering and Applied Science

August 2016 


\section{Abstract}

More than 2.5 billion people lack access to an improved water supply and improved sanitation facilities. Most of them are from lower-income countries (LICs)-where annual municipal budgets for water and sanitation (WatSan) services are highly variable, often inadequate to cover current operation and maintenance, and difficult to use for investment in capital expansion projects. My research aims to provide a budget allocation system for municipal WatSan managers in LICs in the face of severe budget constraints; this budget allocation system aims to protect LICs from public health impacts associated with inadequate water and sanitation services. The process, called Sequential Budget Allocation, requires municipalities to set aside a portion of their annual budget for capacity expansion, and optimizes the allocation

of these funds over the three services (drinking water supply-DWS, wastewater and sewage treatment-WST, and municipal solid waste management-MSW) and across the multiple service regions in the municipality. I first use a systems approach to construct an integrated water and sanitation system model (IWSS), which represents the deficit status of WatSan services in all regions. I use enteric diarrheal disease (EDD) as the indicator of impact of inadequate WatSan services. Then, I propose a nonlinear differential equations model called SIWDR (Susceptible-Infected-WaterDumpsite-Recovered), which links the status of WatSan services to the total incidents of EDD in the service region. Finally, I use optimal control theory to allocate the budget to the three services across the multiple service regions in the municipality in 
a sequence of annual investments designed to minimize the incidence of EDD over a specified planning horizon. With certain budget constraints, the total cases of enteric diseases are minimized in SIWDR. 
To my beloved family 


\section{Acknowledgments}

The writing of this dissertation has been one of the most significant academic challenges of my life. Without the help and guidance of the following people, I would not complete my study.

First of all, I would like to express my deepest gratitude to my advisor and mentor, Dr. Garrick E. Louis, for his support, caring, patience, and providing me with endless opportunities in pursuing my own research. I remember vividly how he welcomed me to his research group with such a warm and powerful speech. He also gave me the opportunity to widen my perspective to visit the real water and sanitation crisis in Siuna, Nicaragua. This experience has been one of the most influential experiences that led me to do dissertation related to water and sanitation. Because of Dr. Garrick E. Louis, I have grown academically and socially.

Secondly, I would like to thank my dissertation advisory committee who guided me through all these years. Dr. Gerard Learmonth often gave me great pieces of advice to get through obstacles. Dr. William T. Scherer opened me up to a new way of thinking-systems thinking. He pushed me to think critically about my own work, and encouraged me to improve my writing. Dr. James H. Lambert was always available when I need help. Finally, Dr. Zoran Grujic, whom I have known since I was an undergraduate student at the University of Virginia, has helped me tremendously with mathematical models. I am really grateful that I have been guided by this committee. 
Another person that I would to thank is Dr. Wirawan Chinviriyasit. She is a brilliant mathematician at the King Mongkut's University of Technology Thonburi, Thailand. Without knowing each other beforehand, she agreed via email to give me advice on how to solve the mathematical problem that I had been struggle for over 6 months, and allowed me to meet with her in person. She introduced me to the new path where my research could reach its full potential.

Special thanks to my family (Paisal Chaysiri, Seangchant Chaysiri, Siriwan Chaysiri, and Sara Samiphak). My family always loves and supports me unconditionally. I would not have been able to get to this point in my life without them. Also, I would like to thank all of my friends who supported me in this writing, and encouraged me to strive towards my goal. 


\section{Contents}

Contents vii

List of Tables . . . . . . . . . . . . . . . . . . ix

List of Figures . . . . . . . . . . . . . . . . . . . . . xi

1 Introduction $\quad 1$

1.1 Background .......................... 1

1.2 Research questions . . . . . . . . . . . . . . . . 5

1.3 Goals and objectives . . . . . . . . . . . . . . 6

1.4 Dissertation outline . . . . . . . . . . . . . . . . . . . . 7

2 Integrated Water and Sanitation System (IWSS) 8

2.1 General description of an IWSS . . . . . . . . . . . . . . . . . . 8

2.2 Adjacency matrix . . . . . . . . . . . . . . . . . . . . . . 12

2.3 IWSS diagrams . . . . . . . . . . . . . . . . . . . . . 13

2.4 Capital investment in WatSan services . . . . . . . . . . . . . . 18

2.5 Summary of an IWSS . . . . . . . . . . . . . . . . . . . . . . 19

3 Compartmental Models 21

3.1 Systems modeling . . . . . . . . . . . . . . . . . . . . . . . 22

3.2 Numerical simulations . . . . . . . . . . . . . . . . . . . . . 29

3.3 WatSan's service impact . . . . . . . . . . . . . . . . 37

3.4 Summary of SIWDR model . . . . . . . . . . . . . . . . . . . 39

4 Fundamental Properties of SIWDR Model 40

4.1 Feasible region of the equilibria of the model . . . . . . . . . . . . . . 40

4.2 Stability of disease-free equilibrium (DFE) . . . . . . . . . . . . . . 42

4.2 .1 Local stability . . . . . . . . . . . . . . . . . . . . . . 42

4.2 .2 Global stability of DFE . . . . . . . . . . . . . . 46

4.3 Endemic equilibrium (EE) . . . . . . . . . . . . . . . . . 47

4.3.1 Existence of endemic equilibrium . . . . . . . . . . . . . . 47

4.3.2 Local stability of endemic equilibrium . . . . . . . . . . . 51

4.4 Summary of the fundamental properties of the SIWDR model . . . . 59 
5 Optimal Control for SIWDR Model $\quad 60$

5.1 Introduction . . . . . . . . . . . . . . . . . 61

5.2 Systems modeling . . . . . . . . . . . . . . . . . . . 63

5.3 Existence of solution of the control system . . . . . . . . . . 65

5.4 Optimal control strategy $\ldots \ldots \ldots \ldots$

5.5 Results and discussion . . . . . . . . . . . . . . . . 73

5.6 Summary of optimal control for SIWDR model . . . . . . . . . . 85

6 Conclusions and Future Research Directions $\quad 87$

6.1 Findings and insights . . . . . . . . . . . . . . 88

6.2 Future research directions $\ldots \ldots \ldots$. . . . . . . . . . . 90

$\begin{array}{ll}\text { Appendix A } & 92\end{array}$

A.1 Assumptions (van den Driessche and Watmough [74]) . . . . . . . . 93

A.2 Theorem 5. (van den Driessche and Watmough [74]) . . . . . . . . 94

A.3 Theorem 6. (van den Driessche and Watmough [74]) . . . . . . . . . 94

$\begin{array}{ll}\text { Appendix B } & 95\end{array}$

B.1 The range explored for parameters from table $3.3 \ldots \ldots \ldots$

$\begin{array}{ll}\text { Appendix C } & 107\end{array}$

C.1 Matlab code: Forward-Backward Sweep Method . . . . . . . . . . 108 


\section{List of Tables}

2.1 State variables of an IWSS. . . . . . . . . . . . . . . . 9

2.2 Input/output WatSan service system. . . . . . . . . . . . . . 11

3.1 Variables for the SIWDR model (3.3), together with sample unit . . . 27

3.2 Parameters for the SIWDR model (3.3) and (3.4) together with sample unit . . . . . . . . . . . . . . . . . . 28

3.3 Parameters used in the SIWDR model (3.3) and (3.4), initial values, range explored, and sources of parameters are shown. Please refer to the Table 3.2 for the meaning of each parameter, as well as its unit. Because budget decision occurs on a monthly basis, I change units of all parameters from days to months. . . . . . . . . . . . . . . 29

3.4 Effect of $r_{1}$ on $R_{0}$ and $I$ at $t=60 \ldots \ldots \ldots \ldots \ldots \ldots \ldots \ldots$

3.5 Effect of $r_{2}$ on $R_{0}$ and $I$ at $t=60 \ldots \ldots \ldots \ldots \ldots \ldots \ldots$

3.6 Effect of $r_{3}$ on $R_{0}$ and $I$ at $t=60 \ldots \ldots \ldots \ldots \ldots \ldots \ldots \ldots \ldots \ldots$

3.7 Cost of WatSan services in LIC. Table includes WatSan services, cost per capita, ranges of cost per capita, technology, and source of the data. 37

5.1 Case 1: Comparison of number of infected population in the situation with no controls, and that with controls. . . . . . . . . 76

5.2 Case 1: Sensitivity analysis of various control parameters. . . . . . . 79

5.3 Case 2: Comparison of number of infected population in the situation with no controls, and that with controls. . . . . . . . . . 80

5.4 Case 2: Sensitivity analysis of various control parameters. . . . . . . 83

5.5 Case 1: Comparison of number of infected population using different strategies. . . . . . . . . . . . . . . . . . . 84

5.6 Case 2: Comparison of number of infected population using different strategies. . . . . . . . . . . . . . . . . . 84

B.1 Effect of $\Lambda$ on $R_{0}$ and $I$ at $t=60 \ldots \ldots \ldots \ldots \ldots$

B.2 Effect of $\mu$ on $R_{0}$ and $I$ at $t=60 \ldots \ldots \ldots \ldots \ldots \ldots \ldots$

B.3 Effect of $\beta_{I}$ on $R_{0}$ and $I$ at $t=60 \ldots \ldots \ldots \ldots \ldots$

B.4 Effect of $\beta_{W}$ on $R_{0}$ and $I$ at $t=60 \ldots \ldots \ldots \ldots \ldots$

B.5 Effect of $\beta_{D}$ on $R_{0}$ and $I$ at $t=60 \ldots \ldots \ldots \ldots \ldots$

B.6 Effect of $\gamma$ on $R_{0}$ and $I$ at $t=60 \ldots \ldots \ldots \ldots \ldots \ldots \ldots$

B.7 Effect of $\alpha_{1}$ on $R_{0}$ and $I$ at $t=60 \ldots \ldots \ldots \ldots \ldots \ldots$ 
B.8 Effect of $\alpha_{2}$ on $R_{0}$ and $I$ at $t=60 \ldots \ldots \ldots \ldots$

B.9 Effect of $\psi$ on $R_{0}$ and $I$ at $t=60 \ldots \ldots$. . . . . . . . . . . . . . 104

B.10 Effect of $\xi_{W}$ on $R_{0}$ and $I$ at $t=60 \ldots \ldots \ldots \ldots$. . . . . . . 105

B.11 Effect of $\xi_{D}$ on $R_{0}$ and $I$ at $t=60 \ldots \ldots \ldots$. . . . . . . 106 


\section{List of Figures}

1.1 A WatSan service, supply-demand deficit. The demand curve is labeled $\mathrm{D}$, and the supply curve is labeled S. . . . . . . . . . . . 2

2.1 Interrelationships among the three services for one region. . . . . . . 14

2.2 Demand flow . . . . . . . . . . . . . . . . . . . . . 14

2.3 Supply flow . . . . . . . . . . . . . . . . . . . . . . . . . . . . . . . . . . . . . . . .

2.4 The IWSS within one region . . . . . . . . . . . . . 15

2.5 The IWSS for three regions . . . . . . . . . . . . . . . 16

2.6 The IWSS for $\mathrm{N}$ regions . . . . . . . . . . . . . . 16

3.1 The SIR Model . . . . . . . . . . . . . . . . . . . 23

3.2 The SIWR Model with $\frac{1}{\xi_{W}}$ represents pathogens lifetime in water reservoir 24

3.3 Similar to the Figure 3.2, but adding level of service for $\operatorname{DWS}\left(r_{1}\right)$ and

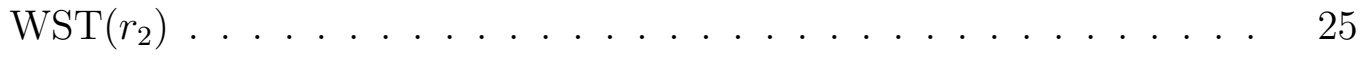

3.4 Flow diagram for the SIWDR model . . . . . . . . . . . . 26

3.5 The SIWDR model simulation. . . . . . . . . . . . . . . . . 31

3.6 The simulation of the SIWDR model using initial values in table 3.3. The dynamics of the number of susceptible and recovered individuals are shown in this figure. . . . . . . . . . . . . . . . . 31

3.7 The simulation of the SIWDR model using initial values in table 3.3. The dynamic of the number of infected individuals is shown in this figure. 32

3.8 The simulation of the SIWDR model using initial values in table 3.3. The culmulative infected population is shown in this figure. . . . . . . 32

3.9 The number of infected population generated from the SIWDR model * (Note: the values of each parameter that is varied from the table 3.3 are as follows: $\beta_{I}=3, \beta_{W}=20, \beta_{D}=30, \alpha_{1}=300$, and $\left.\alpha_{2}=50\right)$. . . .

3.10 Plot shows the number of infected people with various values of parameter $r_{1} \ldots \ldots \ldots \ldots \ldots$

3.11 Plot shows the number of infected people with various values of parameter $r_{2} \ldots \ldots \ldots \ldots \ldots$

3.12 Plot shows the number of infected people with various values of param-

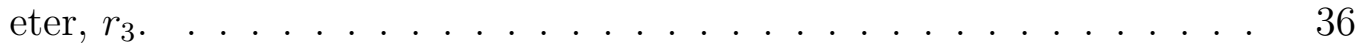

3.13 Comparison of WatSan serivce investment. . . . . . . . . . . . 38

5.1 Lower income countries map. . . . . . . . . . . . . . . . . . . . 62 
5.2 Flow diagram for the SIWDR model . . . . . . . . . . . . 63

5.3 Case 1: Solutions of the SIWDR model with $u_{1}, u_{2}$ and $u_{3} \ldots \ldots$. . . 77

5.4 Case 1: The number of infected population in the eight different situations. 78

5.5 Case 1: The number of infected population in the four different situations that involve $u_{1} \ldots \ldots \ldots$. . . . . . . . . . . . . 78

5.6 Case 2: Solutions of the SIWDR model with $u_{1}, u_{2}$ and $u_{3} \ldots \ldots$. . . 81

5.7 Case 2: The number of infected population in the eight different situations. 82

5.8 Case 2: The number of infected population in the four different situations that involve $u_{1} \ldots \ldots \ldots \ldots$. . . . . . . . . . . 82

6.1 Models used in this study . . . . . . . . . . . . . . . . . . 89

6.2 Flow diagram of the extended SIWDR model . . . . . . . . . . . 91

B.1 Plot shows the number of infected people with various values of parameter, $\Lambda \ldots \ldots \ldots \ldots$. . . . . . . . . . . . . . . 96

B.2 Plot shows the number of infected people with various values of parameter, $\mu \ldots \ldots \ldots \ldots \ldots \ldots$

B.3 Plot shows the number of infected people with various values of parameter, $\beta_{I} \ldots \ldots \ldots \ldots \ldots$

B.4 Plot shows the number of infected people with various values of param-

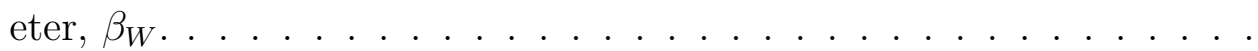

B.5 Plot shows the number of infected people with various values of parameter, $\beta_{D} \ldots \ldots \ldots \ldots$. . . . . . . . . . . . . . . 100

B.6 Plot shows the number of infected people with various values of parameter, $\gamma \ldots \ldots \ldots \ldots \ldots \ldots$

B.7 Plot shows the number of infected people with various values of parameter, $\alpha_{1} \ldots \ldots \ldots \ldots \ldots$

B.8 Plot shows the number of infected people with various values of parameter, $\alpha_{2}$. . . . . . . . . . . . . . . . . . . . . . . . . . . 103

B.9 Plot shows the number of infected people with various values of parameter $\psi$. . . . . . . . . . . . . . . . . . . . . . . . . . . . 104

B.10 Plot shows the number of infected people with various values of parameter, $\xi_{W} \ldots \ldots \ldots \ldots \ldots$

B.11 Plot shows the number of infected people with various values of parameter, $\xi_{D} \ldots \ldots \ldots \ldots \ldots$ 


\section{Chapter 1}

\section{Introduction}

\subsection{Background}

Lack of water and sanitation services is an important problem in lower income countries ${ }^{1}$ (LICs), which results in health, economic, social, and environmental burdens. At least 700 million people lack regular access to safe drinking water and 2.5 billion people live without improved sanitation facilities [84]. The collection of municipal solid waste is usually less than $70 \%$ on average in these countries, and more than $50 \%$ of collected waste is usually dumped in uncontrolled landfills [49] [15]. There is an excess demand for WatSan services in the LICs; while, there is not enough budget to increase the supply of these services (See Figure 1.1 below). Hence, there is a service gap between time t-1 and t. Consequently, capital investment in WatSan services seeks to close this service gap (deficit) over time.

The connections between unclean water, sanitation, and health are well established [58] [24]. Approximately 1.6 million people (90\% of them are under 5 years of age) die every year from diarrheal disease, resulting from the lack of access to WatSan services

\footnotetext{
${ }^{1}$ Lower income countries include countries that have GNI per capita of less than $\$ 4,125$-using the World Bank Atlas method [80]. While the dissertation is specific to communities that lack the resources to provide sustained access to adequate WatSan services, the term lower income countries (LICs) will be used to represent these communities as a whole.
} 


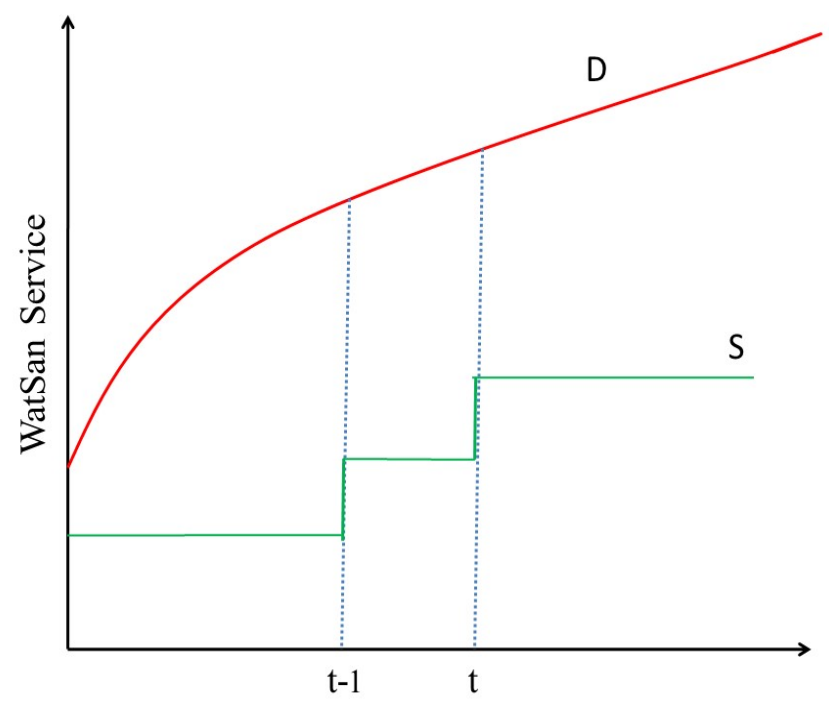

Figure 1.1: A WatSan service, supply-demand deficit. The demand curve is labeled D, and the supply curve is labeled S.

[85]. In addition, a "lack of safe water perpetuates a cycle whereby poor populations become further disadvantaged, and poverty becomes entrenched" [90]. Illnesses caused by contaminated water (e.g. acute respiratory infections, and schistosomiasis [47] [26]) force patients to miss school/work, which results in patients' loss of opportunities and incomes. Furthermore, the time spent collecting safe water elsewhere could be used in other productive activities [35]. In the absence of proper waste management system, discharging wastewater, leachate and sewerage into water sources can directly damage ecosystems.

The lack of access to water and sanitation services has gotten worse in LICs due to high population growth rates that exceeds the rate at which already deficient WatSan service capacity is being expanded in those countries. The lower income countries (LICs) have the world's highest birth rates relative to middle- and higher-income countries [84] [73]. As a result, the demand of WatSan services increases in these countries. Yet, the budget for expanding water and sanitation services is limited. For these LICs, the budget for capacity expansion mainly comes from development aid, which is inconsistent and dependent on donor allocations each year [84]. WatSan is 
a public service, hence WatSan systems are not operated for profit. Therefore, with limited budgets, maximizing revenue of WatSan systems is not a feasible operating objective [45] [22] [76] [91].

Besides budget and high population growth, LICs also lack other capacity factors, such as human resources, institutions, environment, energy, and technical support. This results in the inappropriate management of water and sanitation services [7] [6], causing the supply of WatSan services to decrease even further over time. Thus, the gap between demand and supply of WatSan services keeps widening over time.

In this dissertation, WatSan services refer to the following three services: drinking water supply (DWS), wastewater and sewage treatment (WST), and municipal solid waste (MSW) management. The general description of each service is explained in the Salvato [60] [61]. According to his description, the three services can be explained by their unit processes. Drinking water supply (DWS) service consists of procurement, storage, treatment, and distribution of potable water. This service is linked to individual health. Wastewater and sewage treatment (WST) service consists of collection and treatment of effluents and human waste. This service is linked to individual health and the state of the environment. Municipal solid waste (MSW) service consists of collection and disposal of municipal solid waste, which does not include hazardous waste. This MSW description follows subtitle D of the U.S. Resource Conservation and Recovery Act (RCRA) [71]. However, this dissertation is only concerned with domestic use of water and sanitation services, which excludes other uses, such as agricultural and industrial use.

The integration of DWS, WST, and MSW services into a single water and sanitation system (IWSS) is necessary for effective operation and maintenance (O\&M) of these services over multiple jurisdictions in a service region. Historically, DWS, WST, and MSW services were designed and managed separately. Yet, doing so results in redundancy and high capital and operating costs [44]. Magpili (2004) argued that 
these three basic services share material flows, institutions, finances, industrial, social, and human resources, and organizations [46]. Thus, this research focuses on the integration of DWS, WST, and MSW services over multiple jurisdictions into a single IWSS for strategic capital investment.

Many researchers have integrated DWS, WST, and MSW services for effective capacity expansion. Harremoes (1997) integrated DWS service with WST service, using Material Flow Analysis (MFA) and life cycle analyses of water flow [33]. Louis and Magpili (2007) integrated DWS, WST, and MSW services altogether [43]. Their proposed integrated water and sanitation system proved to be beneficial in terms of "administrative/finance, engineering, and customer service/marketing functions" [44]. They also developed budget allocation strategies for one region, using the deficit analysis based on risk analysis methods [46] [43]. In the US, Polk County in Georgia and Seattle in Washington successfully used integrated WatSan systems to manage their services [56] [64].

However, even with the integration of DWS, WST, and MSW services, it is important to properly manage byproduct materials (i.e., sludge and leachate) in my proposed model. Sludge is generated after the DWS treatment and WST process; while leachate is generated after landfill process in the MSW service. Even small quantities of sludge and leachate can cause many environmental and health problems, because they contain enormous amounts of pathogenic microorganisms [31] [32] [59]. Plus, the situation is worse in the lower income countries where discharging of these byproducts are regulated by weak laws and regulations [66]. Thus, the quantity of sludge and leachate is considered in my model. This is for use in calculating the demand for DWS, WST, and MSW services, for accurate assessment of the status of WatSan services. 


\section{$1.2 \quad$ Research questions}

R1. How can we efficiently increase the supply of WatSan services to match the demand for these services in different subdivisions of a municipal service region? The goal is to provide sustained ${ }^{2}$ access to adequate ${ }^{3}$ water and sanitation services to lower income countries?

R2. What indicators are appropriate for determining the proportions of budget suitable for each service in each region?

R3. What is an effective and equitable strategy for allocating portions of the annual budget to each service in each subdivision over time?

In order to answer these questions, I argue that in order to provide sustained access to WatSan services in the LICs, WatSan's managers first need to understand the interrelationships between water and sanitation services, and how they relate across regions. I construct an integrated water and sanitation system (IWSS) model using a systems approach. This is to assess the WatSan services in all regions as a whole, and determine how water and sanitation services can be improved by the capital investments made through the budget allocations. I use a systems approach to analyze deficits in the WatSan services. Then, I try to reduce these deficits by allocating the annual budget, for each service in each region, in a way that minimizes the total cases of enteric diarrheal diseases (EDD). In this study, I propose to use EDD as the indicator of deficient WatSan services.

Even though, lack of water and sanitation services also results in economic, social,

\footnotetext{
${ }^{2}$ Sustained here means at the scheduled time; at a specific location; and with adequate quantity, and quality over the planning horizon.

${ }^{3}$ Adequacy of water and sanitation services means the quantity, quality, and accessibility of water and sanitation services in accordance with the WHO or the local standards.
} 
and environmental burdens, only the connections between health and unclean water and sanitation are well established [24] [58]. Researcher attempted to analyze the impact of WatSan services on health. Esrey and colleagues examined 144 studies analyzing the impact of WatSan on health. Researchers concluded that improvement in WatSan services can substantially reduce the rates of morbidity and severity of diseases such as diarrhoeal disease [25] [26] [50] [2]. Health impacts are interrelated to economic, environmental, and social impacts. For instance, illnesses force patients to miss schools/work, which results in the loss of incomes. Unhealthy people will not have time to care for the environment around them.

Because deficiencies of DWS, WST, and MSW services result in adverse health effects, I focus on health indicators, more specifically the incidence of EDDs. The EDD is selected because it is the most common disease resulting from the lack of adequate WatSan services, and the most commonly documented disease in official health records [86] [68] [55].

Before undertaking the budget allocation, I examine how the lack of adequate WatSan services can cause EDD in a particular region. I extend a compartmental model, known as SIWR (Susceptible-Infected-Water-Recovered) model. It is a nonlinear differential equation model that is often used in the case of water-borne diseases. I extend SIWR to cover the lack of DWS, WST, MSW services.

\subsection{Goals and objectives}

The goal of this study is to develop a general model that can help with analysis and decision making to increase access to adequate WatSan services in lower income countries (LICs). This goal is achieved through the following three objectives: 
O1. To develop an integrated water and sanitation system (IWSS) model that monitors the deficit in WatSan services over time.

O2. To determine an indicator that represents the impact of the deficit in WatSan services and the effect of capital investment on reducing this deficit in WatSan services over time.

O3. To develop a model to optimize the sequence of capital investments to reduce the WatSan service deficit and its representative impact over the planning horizon.

\subsection{Dissertation outline}

The following is the outline of my dissertation. Chapter 2 provides a general description of an Integrated Water and Sanitation System (IWSS), including the use of IWSS to track the water and sanitation (WatSan) deficit in each region, and the relation of this deficit to capital investment in WatSan services. Chapter 3 proposes the compartmental model called Susceptible-Infected-Water-Dumpsite-Recovered (SIWDR). Chapter 4 shows the fundamental properties of the SIWDR model, and includes mathematical proofs of stability of the disease-free equilibrium and the endemic equilibrium. Chapter 5 introduces an optimal control strategy in the SIWDR model to guide IWSS capital investments across multiple services districts, which yields numerical results. Chapter 6 concludes the dissertation with insights learned regarding the practices in budget allocation for WatSan services, as well as insights that inform the enteric diarrheal disease (EDD) transmission theory. This chapter also includes future research directions. 


\section{Chapter 2}

\section{Integrated Water and Sanitation System (IWSS)}

As mentioned earlier, in LICs, the demand for WatSan services usually exceeds the supply. This results in deficits in DWS, WST, and MSW service [46]. The gap between the demand and supply for each service in each region needs to be monitored. So, I construct a model of an integrated water and sanitation system (IWSS) for this purpose.

\subsection{General description of an IWSS}

An integrated water and sanitation system (IWSS) is composed of three WatSan services (DWS, WST, and MSW) and $N$ service regions. For example, in New York City, DWS, WST, and MSW services must be provided to the five boroughs of the Bronx, Brooklyn, Manhattan, Queens, and Staten Island. In this case $N=5$. These services are managed by municipal WatSan service managers. The general description of IWSS is described below:

Let $i$ be WatSan services for $i=1=\mathrm{DWS}, 2=\mathrm{WST}$, and $3=\mathrm{MSW}$

Let $j$ be the $j^{\text {th }}$ region for $j=1,2, \ldots, N$ 
Let $G_{i j t}$, be the gap between demand and supply of WatSan services in budget interval, t. $G_{i j t}$ can be calculated as:

$$
G_{i j t}=D_{i j t}-S_{i j t}
$$

where

$G_{i j t}$ represents the gap in service, $i$, in region, $j$, at time $t$, $D_{i j t}$ represents the demand for service, $i$, in region, $j$, at time $t$, $S_{i j t}$ represents the supply of service, $i$, in region, $j$, at time $t$, $t$ is the time index for $t=1,2, \ldots, T$-The planning horizon is the capital planning period for the multiple services infrastructure. This may range from a 5-year budget plan for a system in a low-income country to a 50-year capital improvement plan for an independent service authority in a high-income country [63].

$G_{i j t}$ is the state variable of IWSS. It can be displayed in the format below:

Table 2.1: State variables of an IWSS.

\begin{tabular}{|l|l|l|l|l|}
\hline & Region 1 & Region 2 & $\ldots$ & Region N \\
\hline DWS (1) & $G_{11 t}$ & $G_{12 t}$ & $\ldots$ & $G_{1 N t}$ \\
\hline WST $(2)$ & $G_{21 t}$ & $G_{22 t}$ & $\ldots$ & $G_{2 N t}$ \\
\hline MSW $(3)$ & $G_{31 t}$ & $G_{32 t}$ & $\ldots$ & $G_{3 N t}$ \\
\hline Others $(i)^{1}$ & & & & \\
\hline
\end{tabular}

The initial state of IWSS is

$$
\begin{aligned}
G_{i j 0} & =D_{i j 0}-S_{i j 0} \\
& =D_{i j 0_{i n}}+D_{i j 0_{i n t}}-S_{i j 0_{i n}}-S_{i j 0_{o u t}},
\end{aligned}
$$

\footnotetext{
${ }^{1}$ Other services can be considered in the future such as stormwater management (SWM).
} 
where

$G_{i j 0}$ represents the initial gap in service, $i$, in region, $j$, at time 0 ,

$D_{i j 0}$ represents the initial demand for service, $i$, in region, $j$, at time 0 ,

$S_{i j 0}$ represents the initial supply of service, $i$, in region, $j$, at time 0 ,

$D_{i j 0_{i n}}$ represents the demand for service, $i$, in region, $j$, at time 0 ,

$D_{i j 0_{i n t}}$ represents the demand for service, $i$, in region, $j$, at time 0 , obtained from interrelationships among other services within region $j$,

$S_{i j 0_{i n}}$ represents the supply of service, $i$, in region, $j$, at time 0 ,

$S_{i j 0_{\text {out }}}$ represents the supply of service, $i$, in the region, $j$, at time 0 , obtained from interrelationships in service $i$ among other regions outside of region $j$.

Now, before $D_{i j 0_{i n t}}$ and $S_{i j 0_{o u t}}$ can be computed, the regional input/output WatSan service system needs to be established. For $S_{i j 0_{\text {out }}}$, the interrelationships occur when a particular region utilizes/provides WatSan services from/to other regions. For $D_{i j 0_{i n t}}$, the interrelationships occur when an output of a service become an input of other services or vice versa as shown in Table 2.2 below. For example, sludge is an output of both the DWS and WST service components, and becomes an input to the MSW service component when it is treated at MSW facilities. Leachate is an output of the MSW service component, and becomes an input to the WST service component when it is treated at WST plants. Lastly, recycled wastewater is an output of the WST service component, and becomes an input to the DWS service component in some IWSS. A flow of recycled wastewater is assumed to be zero in this study because recycled wastewater is legally prohibited as an input to the DWS service component in most countries.

The interrelationships between the three WatSan services expressed in $D_{i j 0_{i n t}}$ and $S_{i j 0_{\text {out }}}$ can be represented as matrices $I D_{0}$ and $I S_{0}$, respectively (see below). The

\footnotetext{
${ }^{2}$ Only in some countries (e.g., Singapore) with suitable technologies have recycled wastewater. Singapore is a small country with limited water supplies; they recycle wastewater using various technologies such as dual-membrane and ultraviolet disinfection [54].
} 
Table 2.2: Input/output WatSan service system.

\begin{tabular}{|l|l|l|}
\hline & Output System & Input System \\
\hline Recycled Wastewater & WST & DWS \\
\hline Sludge & DWS, WST & MSW \\
\hline Leachate & MSW & WST \\
\hline
\end{tabular}

elements in matrix $I D_{0}$ are 0 if there are no interrelationships between any two WatSan services. Otherwise, elements would equal to $i d_{m n 0}$, which represents the demand for service, $n$, by service, $m$, at time $0 . I S_{0}$ is defined as the interrelationships between supplies across services. Elements in matrix $I S_{0}$ are 0 if there are no interrelationships between the two services. Otherwise, elements would equal to $i s_{m n 0}$, which represents the supply of service, $n$, utilized from service, $m$, at time 0 . This value is positive. Alternatively, $i s_{m n 0}$ is defined as supplies of $m$ service provided to other services. This value is negative. Both matrices are indexed as $D W S_{1}, W S T_{1}, M S W_{1}, D W S_{2}$, $W S T_{2}, M S W_{2}, \ldots, D W S_{N}, W S T_{N}, M S W_{N}$. Here, $N$, is the total number of service regions.

$$
\begin{gathered}
I D_{0}=\left[\begin{array}{ccc}
i d_{110} & \ldots & i d_{1(3 N) 0} \\
\vdots & \ddots & \vdots \\
i d_{(3 N) 10} & \ldots & i d_{(3 N)(3 N) 0}
\end{array}\right] \\
I S_{0}=\left[\begin{array}{ccc}
i s_{110} & \ldots & i s_{1(3 N) 0} \\
\vdots & \ddots & \vdots \\
i s_{(3 N) 10} & \ldots & i s_{(3 N)(3 N) 0}
\end{array}\right]
\end{gathered}
$$


Finally, $D_{i j 0_{\text {int }}}$ and $S_{i j 0_{\text {out }}}$ are calculated by adding up the column of $I D_{0}$ and $I S_{0}$ that represents the demand and supply of $i$ service in the $j^{\text {th }}$ region.

\subsection{Adjacency matrix}

The notion of interrelationships between the three WatSan services is adapted from the concept of adjacency matrices [30]. An adjacency matrix is a square matrix used to represent a finite graph. Each element of a square matrix shows the relationship between pairs of vertices. For a simple labeled graph, let $v_{i}$ and $v_{j}$ be vertex $i$ and vertex $j$, respectively. The element of the square matrix is 0 or 1 in position $\left(v_{i}, v_{j}\right)$, whether $v_{i}$ and $v_{j}$ are adjacent or not. Two examples are illustrated below. The first example is the undirected graph with 3 vertices, which are adjacent. This graph is represented by the matrix under the graph. At position $(1,2),(2,1),(1,3),(3,1),(2$, $3)$, and $(3,2)$, the elements of the matrix are 1 . This matrix is symmetric.

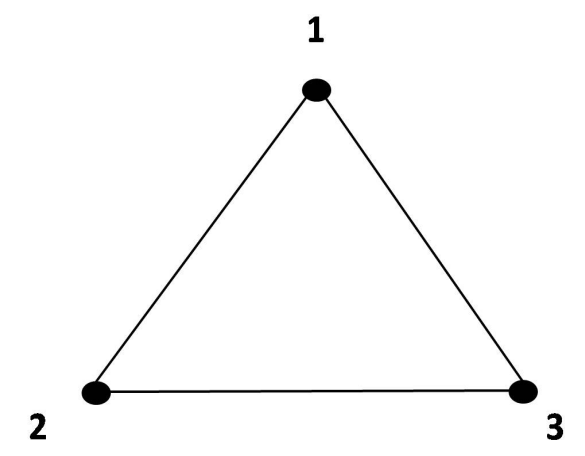

$$
\left(\begin{array}{lll}
0 & 1 & 1 \\
1 & 0 & 1 \\
1 & 1 & 0
\end{array}\right)
$$

Example 1: Undirected graph with 3 vertices 
The second example is the directed graph with 3 vertices, which are adjacent. This graph is represented by the matrix under the graph. At position $(1,2),(1,3)$, and $(2$, $3)$, the elements of the matrix are 1 . This matrix is not symmetric.

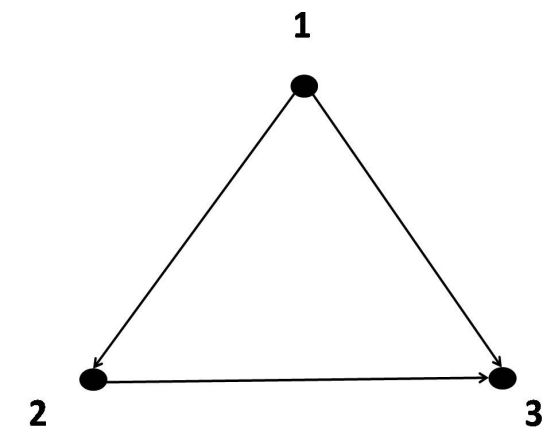

$$
\left(\begin{array}{lll}
0 & 1 & 1 \\
0 & 0 & 1 \\
0 & 0 & 0
\end{array}\right)
$$

Example 2: Directed graph with 3 vertices

The concept of an adjacency matrix is adapted to the IWSS problem and results in the matrix $I D_{0}$ and $I S_{0}$ in the previous section. Instead of 0 or 1 in the position $\left(v_{i}, v_{j}\right), 1$ is replaced by a weight of actual material per unit time when two vertices are adjacent. 0 is left as 0 when two vertices are not adjacent.

\subsection{IWSS diagrams}

For the purpose of visualization, I draw a diagram representing the IWSS below (Figure 2.1). I use vertices and edges [10]. Vertices represent services, and edges represent flow between services. I draw the IWSS's diagram that shows interrelationships among 
services for one region first. The next figures (Figure 2.2 and 2.3) introduce consumers. Solid edges represent demand flows, and dashed edges represent supply flows.

Figure 2.4 combines figures 2.2 and 2.3. This figure demonstrates IWSS within one region.

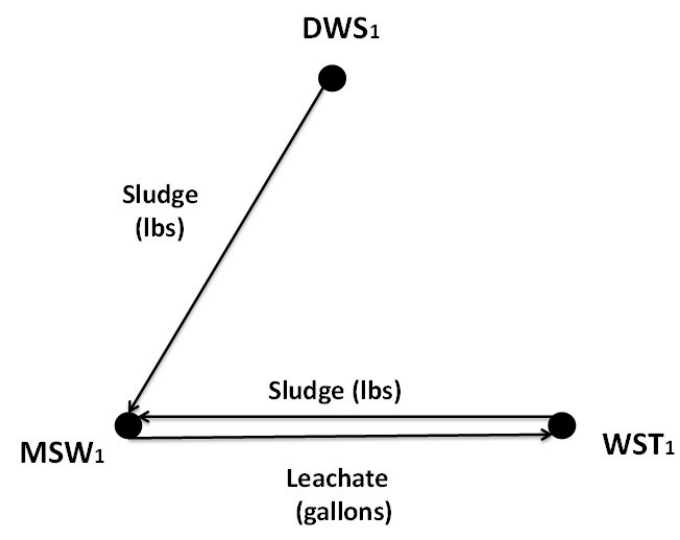

Figure 2.1: Interrelationships among the three services for one region.

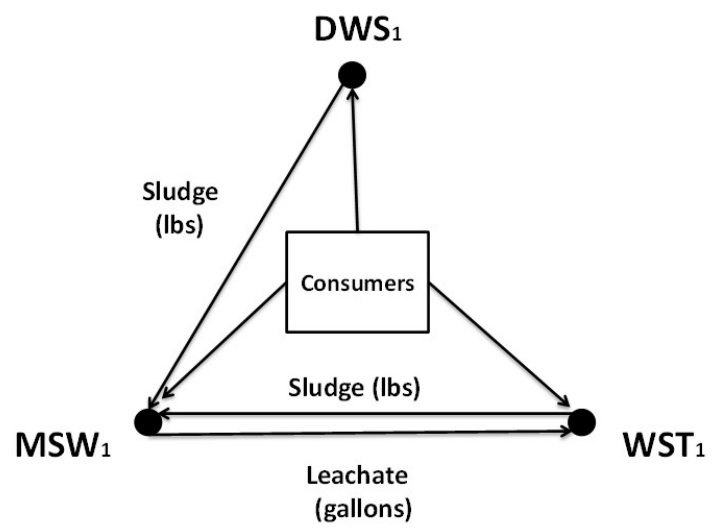

Figure 2.2: Demand flow 


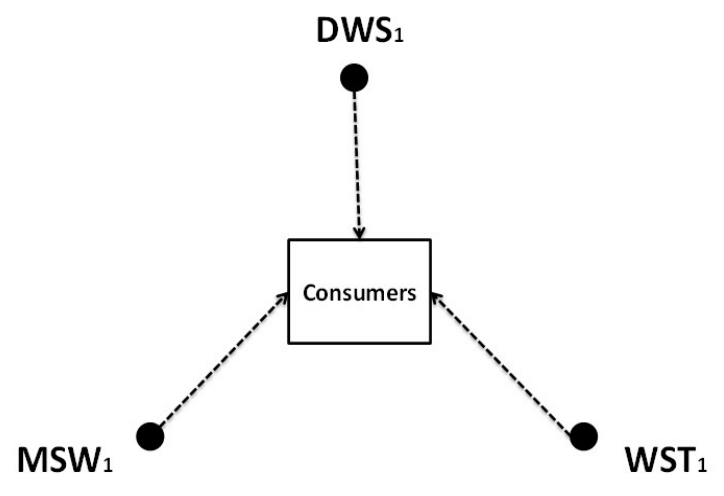

Figure 2.3: Supply flow

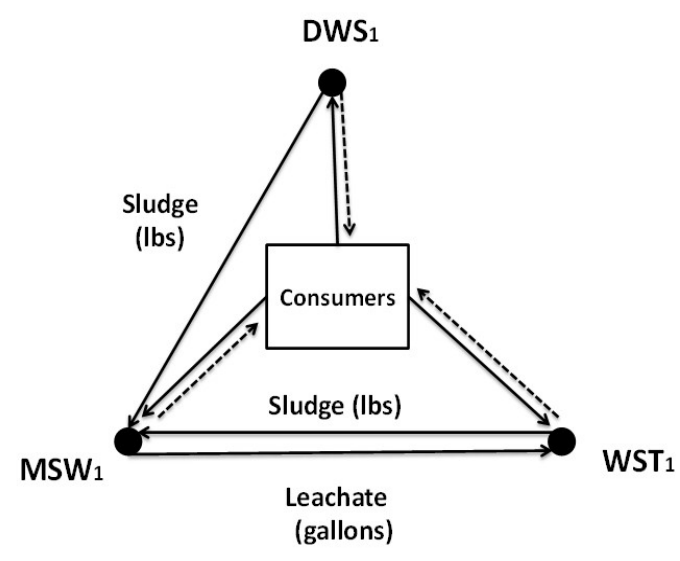

Figure 2.4: The IWSS within one region

Suppose now there are multiple regions. The interrelationships among three regions can be presented below (Figure 2.5). The additional dashed edges are created between $M S W_{1}$ and consumers in region 2 and 3. This means that the supply flows from $M S W_{1}$ located in region 1 are utilized by consumers in region 2 and 3. 


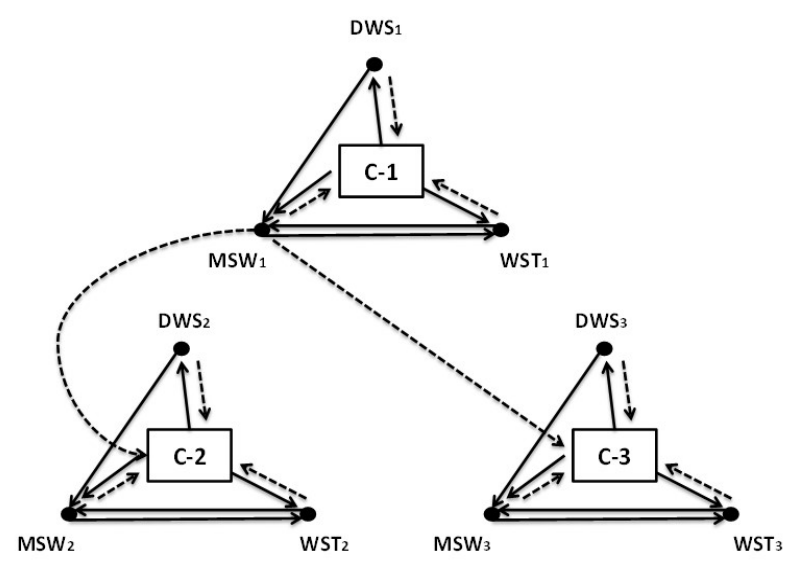

Figure 2.5: The IWSS for three regions

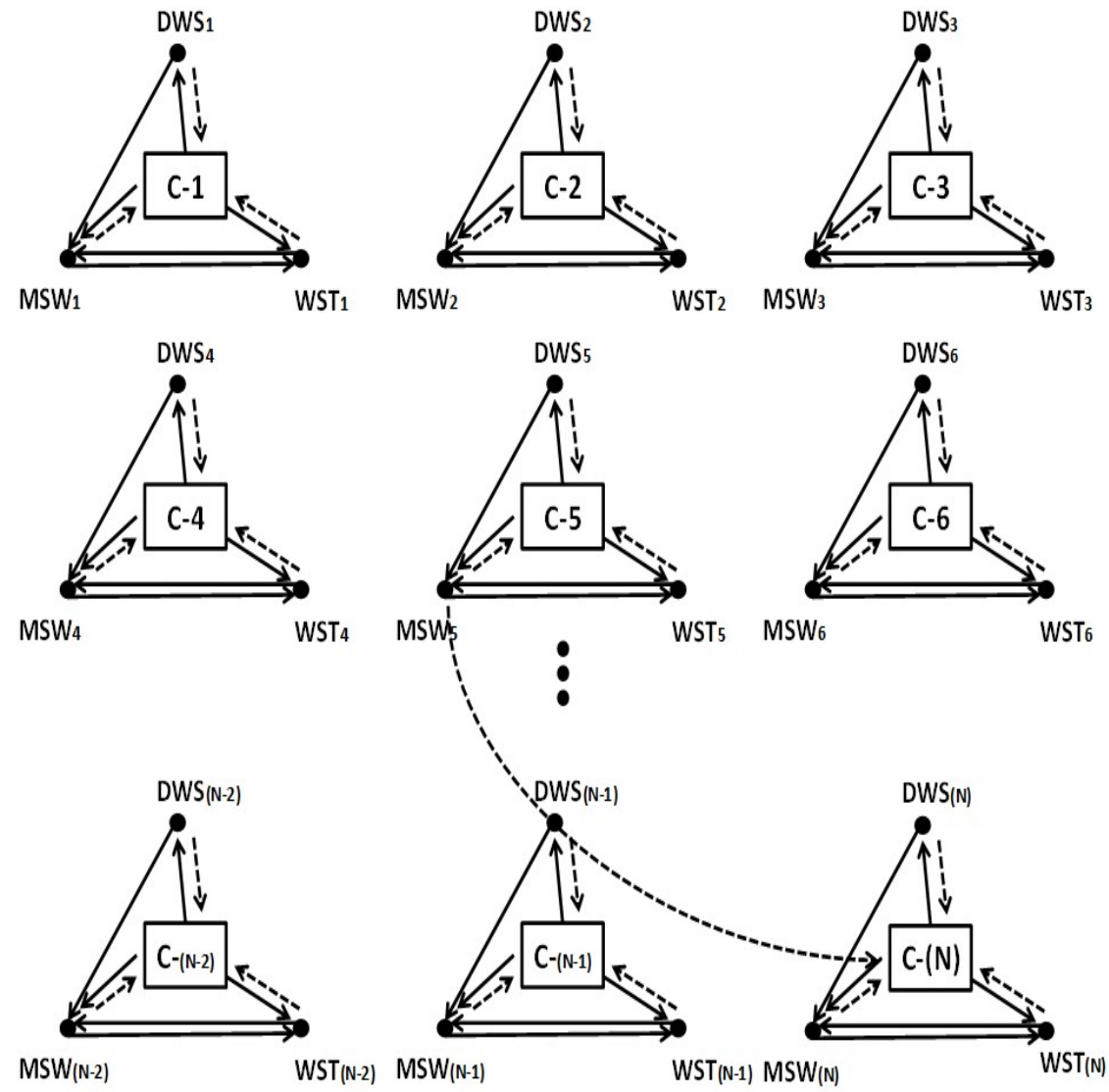

Figure 2.6: The IWSS for N regions 
Suppose $N$ regions are considered. The interrelationships among $N$ regions are presented above. The dashed arrow is created between $M S W_{5}$ and consumers in region $N$ in the Figure 2.6. This means the supply flows from the $M S W_{5}$ located in region 5 is utilized by consumers in region $N$. This case is only one instance for $N$ regions in an IWSS. WatSan managers can draw this kind of diagram to fit their management regions.

Now, I derive equation (2.2) as

$$
\begin{aligned}
G_{i j 0} & =D_{i j 0}-S_{i j 0} \\
& =D_{i j 0_{i n}}+D_{i j 0_{i n t}}-S_{i j 0_{i n}}-S_{i j 0_{o u t}} \\
& =\left(P o p_{j 0} \times d_{i j 0}\right)+\sum_{n} i d_{m(n) 0}-x_{i j 0}-\sum_{n} i s_{m(n) 0},
\end{aligned}
$$

where

$P o p_{j 0}=$ number of population in the region, $j$, at time 0 ,

$d_{i j 0}=$ the demand of service, $i$, per capita, in the region, $j$, at time 0,

$x_{i j 0}=$ the current supply of service, $i$ (service capacity), in the region, $j$, at time 0 .

I am interested in capacity expansion of WatSan services. This expansion factor is represented by population growth and capacity investment. I then assume that other factors causing the change in state variables are constant.

If there is a capacity investment at time $t$, then the state variables can be written as:

$$
G_{i j(t+1)}=G_{i j(t)}-U_{i j(t)}+V_{i j(t)}+W_{i j(t)}
$$

where

$G_{i j(t+1)}$ represents the gap of service, $i$, in the region, $j$, at time $t+1$, $G_{i j(t)}$ represents the gap of service, $i$, in region, $j$, at time $t$, 
$U_{i j(t)}$ represents the capacity expansion from capital investment (add service), $V_{i j(t)}$ represents the increasing in demand resulting from population growth, $W_{i j(t)}$ represents the increasing in demand resulting from capacity investment in other services (an indirect impact),

$t$ is the time index for $t=1,2, \ldots, T$-planning horizon.

\subsection{Capital investment in WatSan services}

Now, I ask "what is the capacity expansion of each service needed to attain the desired state variable, $G_{i j(t+1)}$ ?". This question is a typical question asked by WatSan managers during water and sanitation planning. Now, I have to solve the equation (2.4) for $U_{i j(t)}$.

$$
\begin{aligned}
G_{i j(t+1)} & =G_{i j(t)}+U_{i j(t)}-V_{i j(t)}-W_{i j(t)} \\
U_{i j(t)} & =G_{i j(t)}-G_{i j(t+1)}-V_{i j(t)}-W_{i j(t)}
\end{aligned}
$$

The next step is to ask how much budget is needed to attain the desired state variable, $G_{i j(t+1)}$. This information can be used in WatSan planning.

The process above can be done when the budget is unlimited. But if there is a budget constriant, then how can WatSan managers simultaneously allocate budget to each service in each region?

Finally, the impact of each WatSan service is used to determine the proportion of the budget to allocate to each service, respectively. WatSan managers would allocate the budget in the way that achieves the highest influence to the communities. Each WatSan service yields different type of impacts such as human health, environmental, and economic, which are difficult to estimate directly, relative to other WatSan Services. In addition, data relating to all impacts is scarce. In this research, I use a health impact as a proxy for WatSan's impacts because the health impact is 
relatively well documented in official health records unlike environmental and economic impacts, which are often not available. I use the incidence of enteric diarrheal disease (EDD) as the health indicator as this is a commonly recorded statistics. The gap of WatSan services, $G_{i j t}$, will then be linked to the incidence of EDD through utilizing the compartmental model. I will then determine the combination of capital investment within WatSan services that best minimize the incidence of EDD.

\subsection{Summary of an IWSS}

This chapter quantitatively analyzes water and sanitation services in terms of demand and supply of services. The IWSS is an extension of the conceptual model of water and sanitation systems (Magpili, 2003) [46]: Whereas Magpili assumed a single aggregate region, this IWSS model is able to capture multiple regions at once and demonstrate interrelationships between WatSan services. In addition, the IWSS is used to track the water and sanitation (WatSan) deficit in each region. The IWSS aims to improve water and sanitation service in lower income countries. WatSan managers can use the IWSS to evaluate water and sanitation service system across regions as a whole. This would allow them to allocate resources across regions more efficiently, thereby reducing the mismanagement of funds and improving overall access to WatSan services.

After the IWSS is developed, I am interested in the budget allocation to expand WatSan services across multi-region. The difficulty of tracking the multi-region sequential budget allocation (Magpili, 2003) can be summarized as follows:

i) WatSan managers may subjectively choose certain impacts of inadequate WatSan services.

ii) WatSan managers have to calculate the impacts of the three services (DWS, WST, and MSW service) in multiple different regions, i.e., for every service and every region. This gets harder if many regions are involved, and allows 
errors to propagate throughout the process.

iii) WatSan managers need to calculate new impacts every time a new plan occurs.

iv) In addition, after the investment, changes might be hard to calculate, which causes the calculation of later impacts of inadequate WatSan services to be imprecise.

For the remainder of this dissertation, I am going to develop the model called SIWDR (Susceptible-Infected-Water-Dumpsite-Recovered), which links the status of WatSan services to the total incidents of enteric diarrheal disease (EDD). Then, I will use optimal control theory to find optimal strategies designed to minimize the incidence of EDD over a specified planning horizon. This will help to eliminate the difficulty of tracking the multi-region budget allocation above. 


\section{Chapter 3}

\section{Compartmental Models}

The goal of this research is to develop and test a model for reducing the deficit in water and sanitation services (WatSan) in resource-constrainted communities in order to reduce the incidence of enteric diarrheal disease (EDD). The connections between unclean water, sanitation, and health are well established [58] [24] [85] [47] [26]. Approximately 1.6 million people (90\% of them are under 5 years of age) die every year from diarrheal disease, resulting from the lack of access to WatSan services [85]. The rationale is that increasing WatSan services will reduce the incidence of EDD, and this reduction is an indicator of improved access to WatSan services. The WatSan services include drinking water supply (DWS), wastewater and sewage treatment (WST), and municipal solid waste management (MSW). Relatedly, I want to develop a systematic way to allocate the annual budget across these three services, in order to achieve the greatest reduction in the number of the infected individuals. In this research, I am interested in enteric pathogens associated with diarrhea in most developing countries.

The enteric pathogens can be spread through many pathways from fecal matter to humans by ingestion. For instance, the pathogens can be passed from the feces of infected people to the surrounding environment, and finally to healthy individuals 
through water and food. The pathways are called fecal-oral disease transmission (fecaloral routes). The transmission of disease can be stopped by cutting the transmission pathways. DWS, WST, and MSW services are added for this purpose. According to Keusch (2006), improving DWS and WST services is an effective means of reducing the number of infected individuals and the number of people who die each year as a result of diarrhea [38]. However, MSW service is also taken into account in this case, because there is limited formal management of MSW in many lower income countries. Consequently, dumpsites ${ }^{1}$ can be one of the most important and largest reservoirs of pathogens in these communities. Pathogens in the dumpsites can get into water sources through leachate, which contaminates unprotected groundwater sources or through precipitation that washes into surface water [40] [28]. On the other hand, individuals can be infected if they come near the dumpsite and contact the waste directly [42] [1] [62] [29]. This is common among waste pickers who make their living retrieving waste items from dumpsites for the purpose of recycling. Sequential budget allocations to improve access to DWS, WST, and MSW services affect the incidence of WatSan-related diseases, represented by EDD.

\subsection{Systems modeling}

Starting with the SIWR (Susceptible-Infected-Water-Recovered) model [69] which is built up on the SIR (Susceptible-Infected-Recovered) model invented by Kermack and Mckendrick (1927), the ordinary differential equations (ODE) are written to express the relations between the improvement of water and sanitation services and the rate of infection [37]. I start with the SIR model that has three compartments as in Figure 3.1. The three compartments are:

\footnotetext{
${ }^{1}$ informal solid waste sanitation sites.
} 


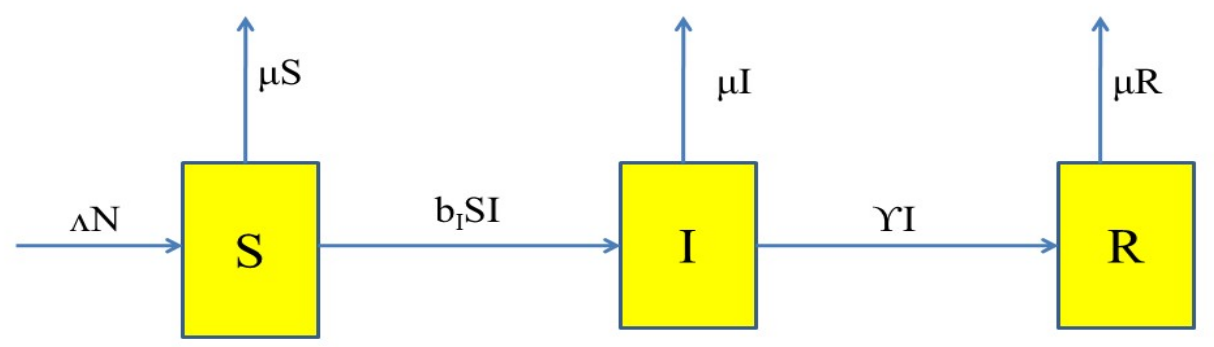

Figure 3.1: The SIR Model

1. Susceptible $(S)$ represents individuals who may become infected if they have contact with an infected individual.

2. Infected $(I)$ represents individuals who are currently infected and can spread the disease to $S$ whom they contact.

3. Recovered $(R)$ represents individuals who are previously infected, but are currently recovered.

Let $N=S+I+R$ be the total population size, $\Lambda$ be birth rate, $\mu$ be mortality rate, $b_{I}$ be the transmission rate (per capita), and $\gamma$ be recovery rate. $S, I$, and $R$ are treated as continuous variables, and express the model in differential equations:

$$
\begin{aligned}
\dot{S} & =\Lambda N-b_{I} S I-\mu S \\
\dot{I} & =b_{I} S I-\gamma I-\mu I \\
\dot{R} & =\gamma I-\mu R
\end{aligned}
$$

where a dot $(\cdot)$ represents differentiation with respect to time $(t)$. 


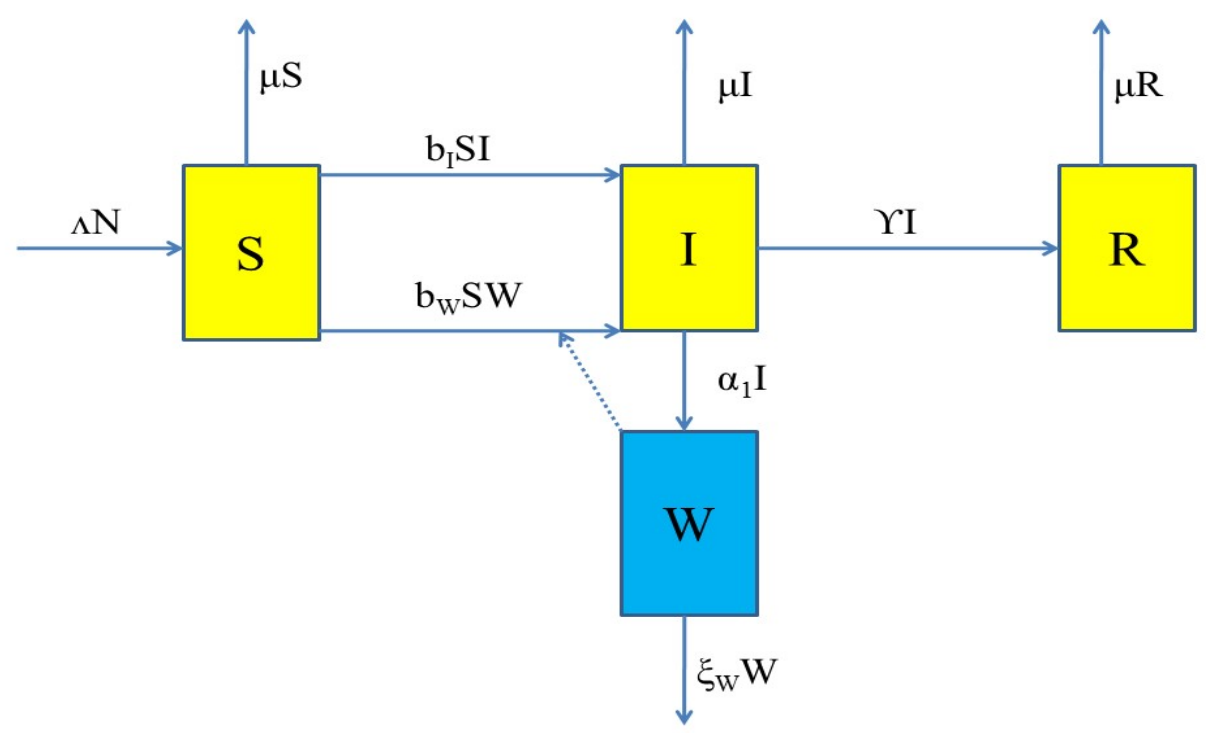

Figure 3.2: The SIWR Model with $\frac{1}{\xi_{W}}$ represents pathogens lifetime in water reservoir

Tien and Earn (2010) introduced the SIWR model, which is obtained by adding compartment $W$ [69]. This model is used for waterborne disease transmissions. Line $\alpha_{1} I$ assumes the pathogens can be passed to water by infected individuals (shedding). Now, if individuals use this infected water for food or for drinking, they will be infected. This is represented by the rate of infection $b_{W} S W$. Alternatively, the model can be expressed in differential equations:

$$
\begin{aligned}
\dot{S} & =\Lambda N-b_{W} S W-b_{I} S I-\mu S \\
\dot{I} & =b_{W} S W+b_{I} S I-\gamma I-\mu I \\
\dot{W} & =\alpha_{1} I-\xi_{W} W \\
\dot{R} & =\gamma I-\mu R
\end{aligned}
$$

Now, what happens to the model if DWS $\left(r_{1}\right)$ and WST $\left(r_{2}\right)$ services are increased? Given that $r_{1}$ and $r_{2}$ are representative of the ratio of people who received DWS and WST services, respectively, in a community: 
(i) If there are no DWS and WST services, $r_{1}$ and $r_{2}$ will be equal to zero, i.e., the figure 3.2 .

(ii) If $r_{1}$ is increased, the rate of infected individuals (susceptible individuals turning into infected ones) will be decreased because the rate is equal to $\left(1-r_{1}\right) b_{W} S W$.

(iii) If $r_{2}$ is decreased, the rate of pathogens getting into water from infected individuals $\left(\left(1-r_{2}\right) \alpha_{1} I\right)$ will be decreased.

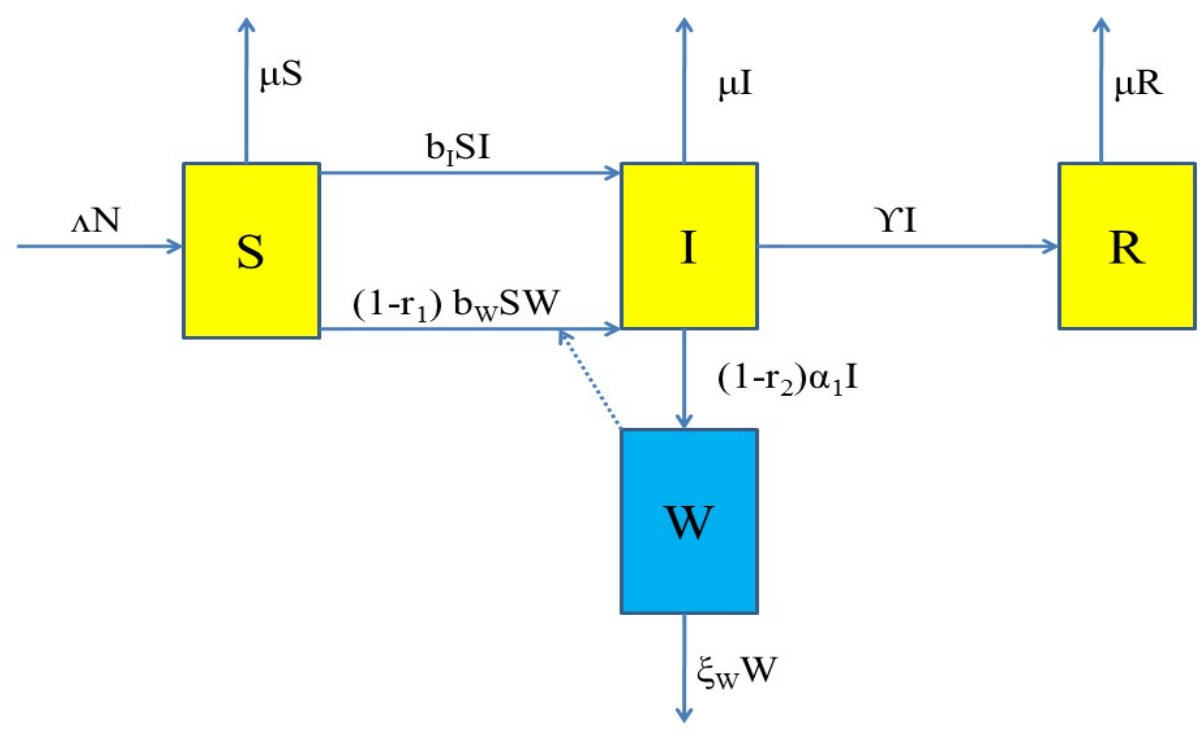

Figure 3.3: Similar to the Figure 3.2, but adding level of service for $\operatorname{DWS}\left(r_{1}\right)$ and $\mathrm{WST}\left(r_{2}\right)$

Compartment $D$ is added in the figure 3.4 below. $D$ represents informal solid waste sanitation sites (dumpsites), which are potential reservoirs of pathogens. Similarly, $r_{3}$ are representative of the ratio of those who received MSW services to the total population in a community. This model is called the SIWDR (Susceptible-InfectedWater-Dumpsite-Recovered) model. Three things to denote: 
(i) The compartment $D$ is related to $S$ because susceptible individuals can come to contact with the infected waste directly. This is represented by $\left(1-r_{3}\right) b_{D} S D$.

(ii) The compartment $D$ is also related to $W$, and this is represented by $\left(1-r_{3}\right) \psi D$.

(iii) It can be noted that if MSW service is increased, $r_{3}$ will be increased. Thus, the rate of infected individuals $\left(\left(1-r_{3}\right) b_{D} S D\right)$ is decreased. Also, the rate of pathogens getting into the surroundings $\left(\left(1-r_{3}\right) \psi D\right)$ is decreased.

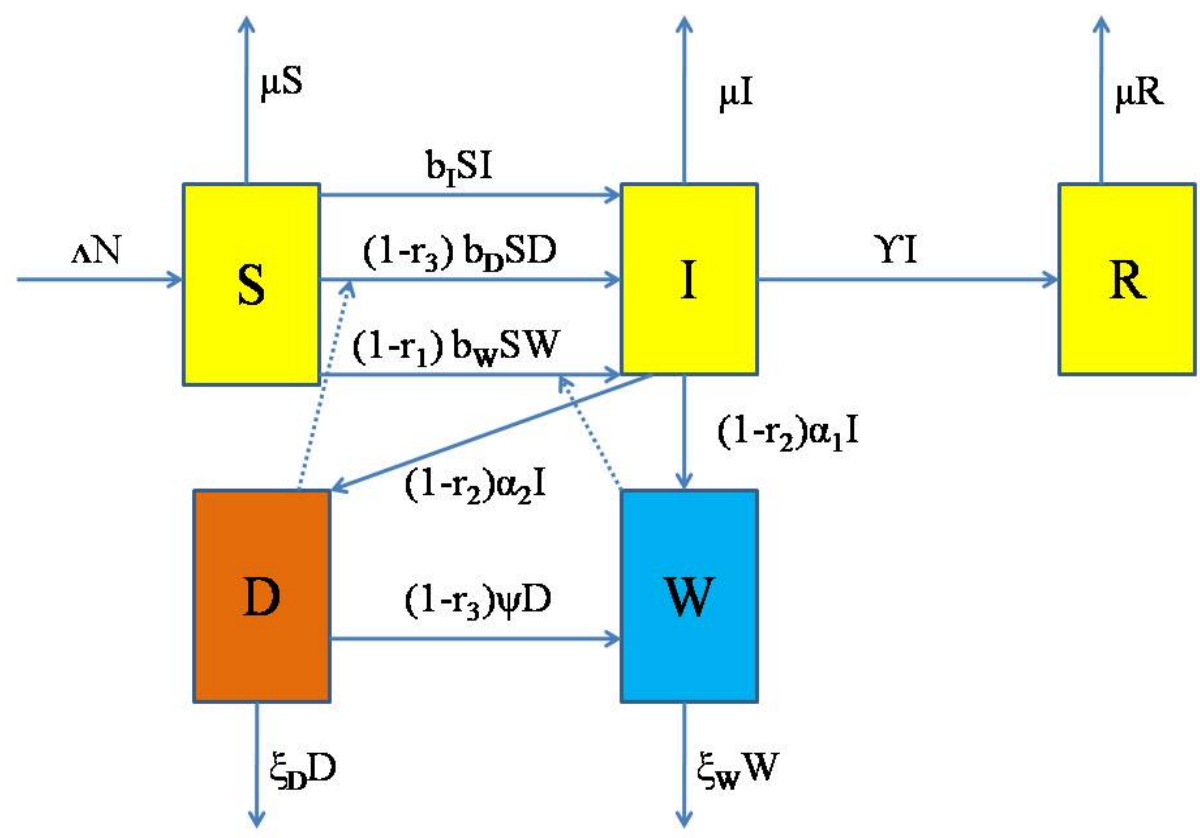

Figure 3.4: Flow diagram for the SIWDR model

Three transmission pathways can occur within the SIWDR model: person-toperson, person-to-water, and person-to-dumpsite. The SIWDR model is used to explain pathways of infection in waterborne diseases. Person-to-person rarely occurs (i.e., zero transmission) among waterborne diseases, but it can occur in cholera. 
The system of nonlinear differential equations is

$$
\begin{aligned}
\dot{S} & =\Lambda N-\left(1-r_{1}\right) b_{W} S W-\left(1-r_{3}\right) b_{D} S D-b_{I} S I-\mu S \\
\dot{I} & =\left(1-r_{1}\right) b_{W} S W+\left(1-r_{3}\right) b_{D} S D+b_{I} S I-\gamma I-\mu I \\
\dot{W} & =\left(1-r_{2}\right) \alpha_{1} I+\left(1-r_{3}\right) \psi D-\xi_{W} W \\
\dot{D} & =\left(1-r_{2}\right) \alpha_{2} I-\xi_{D} D \\
\dot{R} & =\gamma I-\mu R
\end{aligned}
$$

Table 3.1: Variables for the SIWDR model (3.3), together with sample unit

\begin{tabular}{|l|l|l|}
\hline & Description & Sample unit \\
\hline$S$ & susceptible individual density & individuals $\mathrm{km}^{-2}$ \\
\hline$I$ & infected individual density & individuals $\mathrm{km}^{-2}$ \\
\hline$R$ & recovered individual density & individuals $\mathrm{km}^{-2}$ \\
\hline$W$ & pathogen concentration in water reservoir & cells $\mathrm{ml}^{-1}$ \\
\hline$D$ & pathogen concentration in dumpsite & cells $\mathrm{kg}^{-1}$ \\
\hline$N$ & total population density & individuals $\mathrm{km}^{-2}$ \\
\hline
\end{tabular}

For ease of analysis, the system model (3.3) is scaled to be dimensionless variables. Let $N$ denote the total population size, $N=S+I+R$.

Also let

$$
s=\frac{S}{N}, \quad i=\frac{I}{N}, \quad r=\frac{R}{N}, \quad w=\frac{\xi_{W}}{\alpha_{1} N} W, \quad d=\frac{\xi_{D}}{\alpha_{2} N} D .
$$

The transmission parameters are replaced by

$$
\beta_{I}=b_{I} N, \quad \beta_{W}=\frac{b_{W} \alpha_{1} N}{\xi_{W}}, \quad \beta_{D}=\frac{b_{D} \alpha_{2} N}{\xi_{D}}
$$

\footnotetext{
${ }^{2}$ cells or colony-forming units.
} 
Then, the scaled version of the system model (3.3) is as following.

$$
\begin{aligned}
\dot{s} & =\Lambda-\left(1-r_{1}\right) \beta_{W} s w-\left(1-r_{3}\right) \beta_{D} s d-\beta_{I} s i-\mu s \\
\dot{i} & =\left(1-r_{1}\right) \beta_{W} s w+\left(1-r_{3}\right) \beta_{D} s d+\beta_{I} s i-\gamma i-\mu i \\
\dot{w} & =\xi_{W}\left(1-r_{2}\right) i+\frac{\left(1-r_{3}\right) \alpha_{2} \xi_{W} \psi}{\alpha_{1} \xi_{D}} d-\xi_{W} w \\
\dot{d} & =\xi_{D}\left(1-r_{2}\right) i-\xi_{D} d \\
\dot{r} & =\gamma i-\mu r
\end{aligned}
$$

\begin{tabular}{|c|c|c|}
\hline Parameter & Description & Sample unit \\
\hline$b_{I}$ & person-person contact rate & $\mathrm{km}^{2}$ individuals $^{-1}$ day $^{-1}$ \\
\hline$\beta_{I}$ & scaled person-person contact rate & $d a y^{-1}$ \\
\hline$b_{W}$ & water-person contact rate & $m l$ cells $^{-1}$ day $^{-1}$ \\
\hline$\beta_{W}$ & scaled water-person contact rate & $d a y^{-1}$ \\
\hline$b_{D}$ & dumpsite-person contact rate & $k g$ cells $^{-1}$ day $^{-1}$ \\
\hline$\beta_{D}$ & scaled dumpsite-person contact rate & $d a y^{-1}$ \\
\hline$\gamma^{-1}$ & infectious period & day \\
\hline$\xi_{W}^{-1}$ & pathogen lifetime in water & day \\
\hline$\xi_{D}^{-1}$ & pathogen lifetime in dumpsite & day \\
\hline$\alpha_{1}$ & person-water contact rate "sheding" & cells $\mathrm{ml}^{-1}$ day $^{-1} \mathrm{~km}^{2}$ individuals ${ }^{-1}$ \\
\hline$\alpha_{2}$ & person-dumpsite contact rate & cells $\mathrm{kg}^{-1}$ day $^{-1} \mathrm{~km}^{2}$ individuals ${ }^{-1}$ \\
\hline$\psi$ & dumpsite-water contact rate & $k g m l^{-1} d a y^{-1}$ \\
\hline$\Lambda$ & birth rate & $d a y^{-1}$ \\
\hline$\mu$ & death rate & $d a y^{-1}$ \\
\hline$r_{1}$ & fraction of DWS coverage & - \\
\hline$r_{2}$ & fraction of WST coverage & - \\
\hline$r_{3}$ & fraction of MSW coverage & - \\
\hline
\end{tabular}

Table 3.2: Parameters for the SIWDR model (3.3) and (3.4) together with sample unit

The system model (3.4) is mathematically and epidemiologically well-posed and it is sufficient to consider the dynamics of the flow generated by the system model (3.4). Please refer to the chapter 4 for fundamental properties of the SIWDR model. 


\subsection{Numerical simulations}

Table 3.3: Parameters used in the SIWDR model (3.3) and (3.4), initial values, range explored, and sources of parameters are shown. Please refer to the Table 3.2 for the meaning of each parameter, as well as its unit. Because budget decision occurs on a monthly basis, I change units of all parameters from days to months.

\begin{tabular}{|l|l|l|l|}
\hline Parameter & Initial value & Range explored & Source \\
\hline$\Lambda$ & 0.0018 & $0-0.0037$ & CIA World Factbook (2016) [16], [17] \\
\hline$\mu$ & 0.00064356 & $0-0.0012$ & CIA World Factbook (2016) [16], [18] \\
\hline$\beta_{I}$ & 0.5 & $0.3-3$ & Tuite (2011) [70], Eisenberg (2013) [23] \\
\hline$\beta_{W}$ & 2 & $0.0003-30$ & $\begin{array}{l}\text { Tuite (2011) [70], Eisenberg (2013) [23] } \\
\text { Fung (2014) [27] }\end{array}$ \\
\hline$\beta_{D}$ & 4.49 & $0.0003-30$ & - \\
\hline$\gamma$ & 1.5 & $1.5-30$ & $\begin{array}{l}\text { Tuite (2011) [70], King (2008) [39], } \\
\text { WHO (2012) [89], Eisenberg (2013) [23] }\end{array}$ \\
\hline$\alpha_{1}$ & 10 & $0.3-300$ & $\begin{array}{l}\text { King (2008) [39], Hendrix (1971) [34] } \\
\text { Fung (2014) [27] }\end{array}$ \\
\hline$\alpha_{2}$ & 25 & $0.3-300$ & - \\
\hline$\psi$ & 50 & $0.3-300$ & - \\
\hline$\xi_{W}$ & 1.4 & $0.6857-10$ & Tuite (2011) [70], Fung (2014) [27] \\
\hline$\xi_{D}$ & 2 & $0.3477-12$ & UNICEF (2013) [72] \\
\hline$r_{1}$ & 0.635 & $0.0-1.0$ & WHO (2012) [88], WHO (2015) [87] \\
\hline$r_{2}$ & 0.225 & $0.0-1.0$ & WHO (2014) [88], WHO (2015) [87] \\
\hline$r_{3}$ & 0.1 & $0.0-1.0$ & Bras (2009) [8] \\
\hline
\end{tabular}

This section presents numerical simulations of the system model (3.4). Values of parameters are taken from relevant literature. However, the values of $\beta_{D}, \alpha_{2}$, and $\psi$ result from my own estimation. The values of $\beta_{D}, \alpha_{2}$, and $\psi$ are not estimated in the 
literature. Thus, I use the plausible range bounded by other values known from the literature. The value of $\beta_{D}$ is assumed to be in the same plausible range as that of $\beta_{W}$. Similarly, the values of $\alpha_{2}$ and $\psi$ are assumed to be in the same plausible range as that of $\alpha_{1}$.

The assumed initial values are suited for cholera infection in Haiti in 2010 to 2015 [55]. I tune parameters by varying parameters within their plausible ranges to obtain the set of initial values that has the basic reproduction number, $R_{0}{ }^{3}$ (Refer to the chapter 4 for the fundamental properties of the SIWDR model) falls within the plausible range of $R_{0}$ [70] [52]. $R_{0}$ equals to 2.135 for this initial set of parameters. This value is selected because it equals to the average of the basic reproduction number of cholera infection in Haiti. Once all values are set, I will later use this initial set of parameters to illustrate the dynamics of all compartments.

The comparison between SIWDR model simulation and the incidence of cholera in Haiti in 2010-2015 is shown below.

In the figure below, I scale down the simulation of SIWDR model, so that the number of cholera infected population is close to the real number of infected population each month in Haiti.

Notice that the dynamic of the number of infected population is similar to that occurs in reality in Haiti. The number of infected population starts from a small number. Then, it gradually increases to the maximum value, and drops to a constant value, which is not equal to zero. That is, there are some people who are infected with cholera in Haiti.

The graphs below are the numerical simulation of the SIWDR model using initial values shown in the table 3.3. Three compartments (Susceptible, Infected, and Recovered) are shown here in the figure 3.6 and 3.7. The figure 3.8 shows the cumulative infected population.

\footnotetext{
${ }^{3}$ The basic reproduction number, $R_{0}$, is defined as the expected number of cases that one infected individual can produce by infecting other susceptible individuals during its infective period [74].
} 


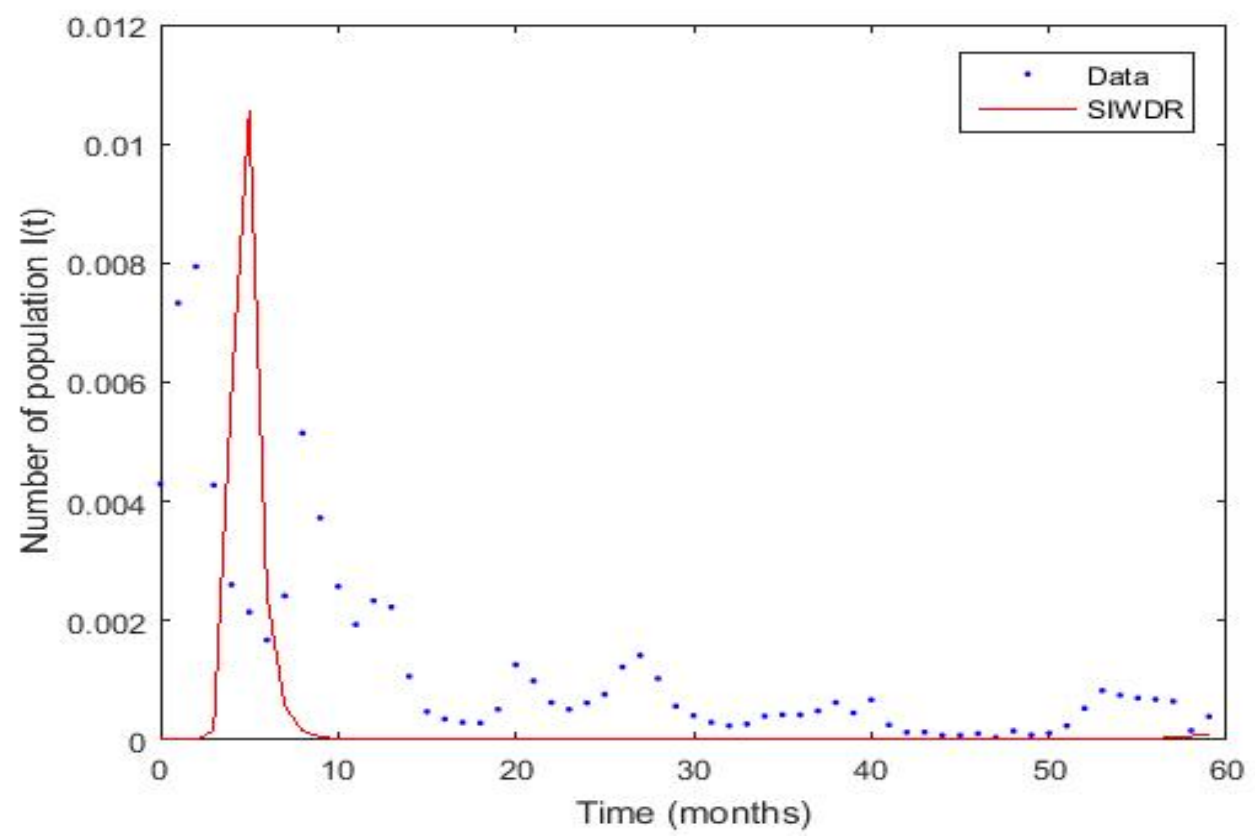

Figure 3.5: The SIWDR model simulation.

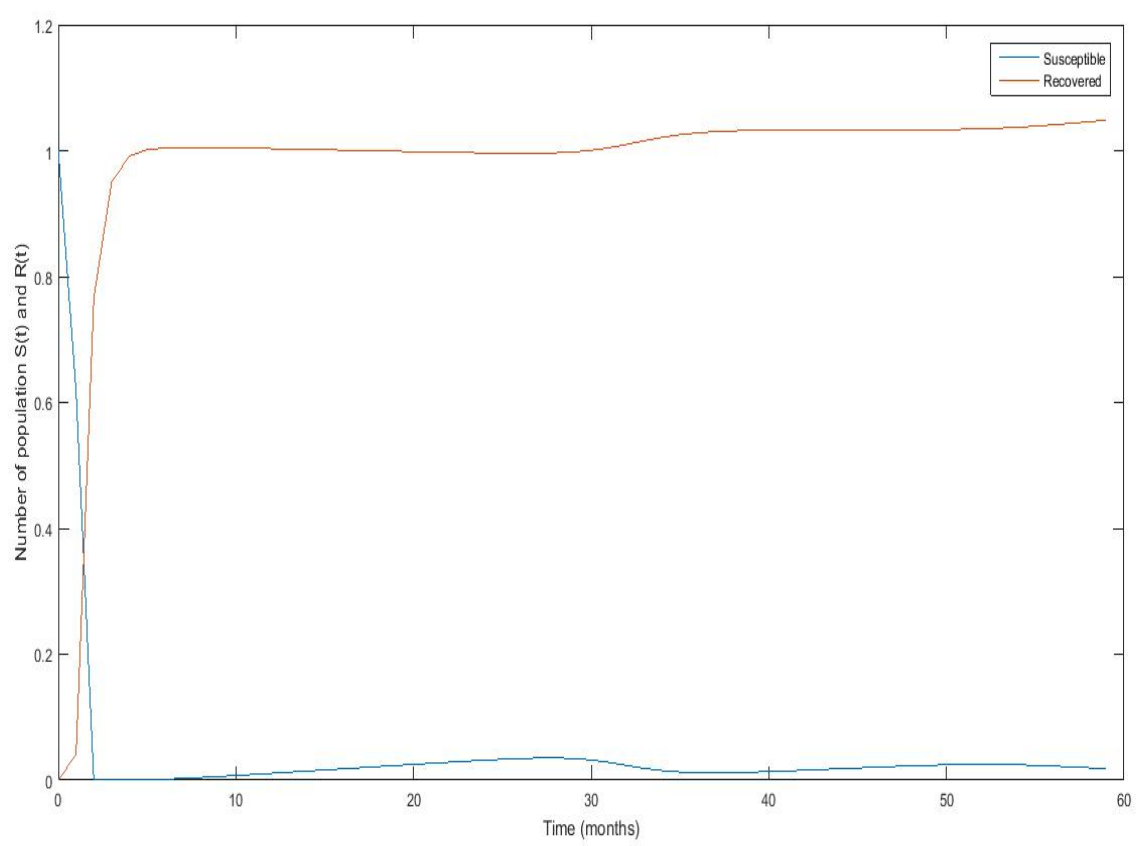

Figure 3.6: The simulation of the SIWDR model using initial values in table 3.3. The dynamics of the number of susceptible and recovered individuals are shown in this figure. 


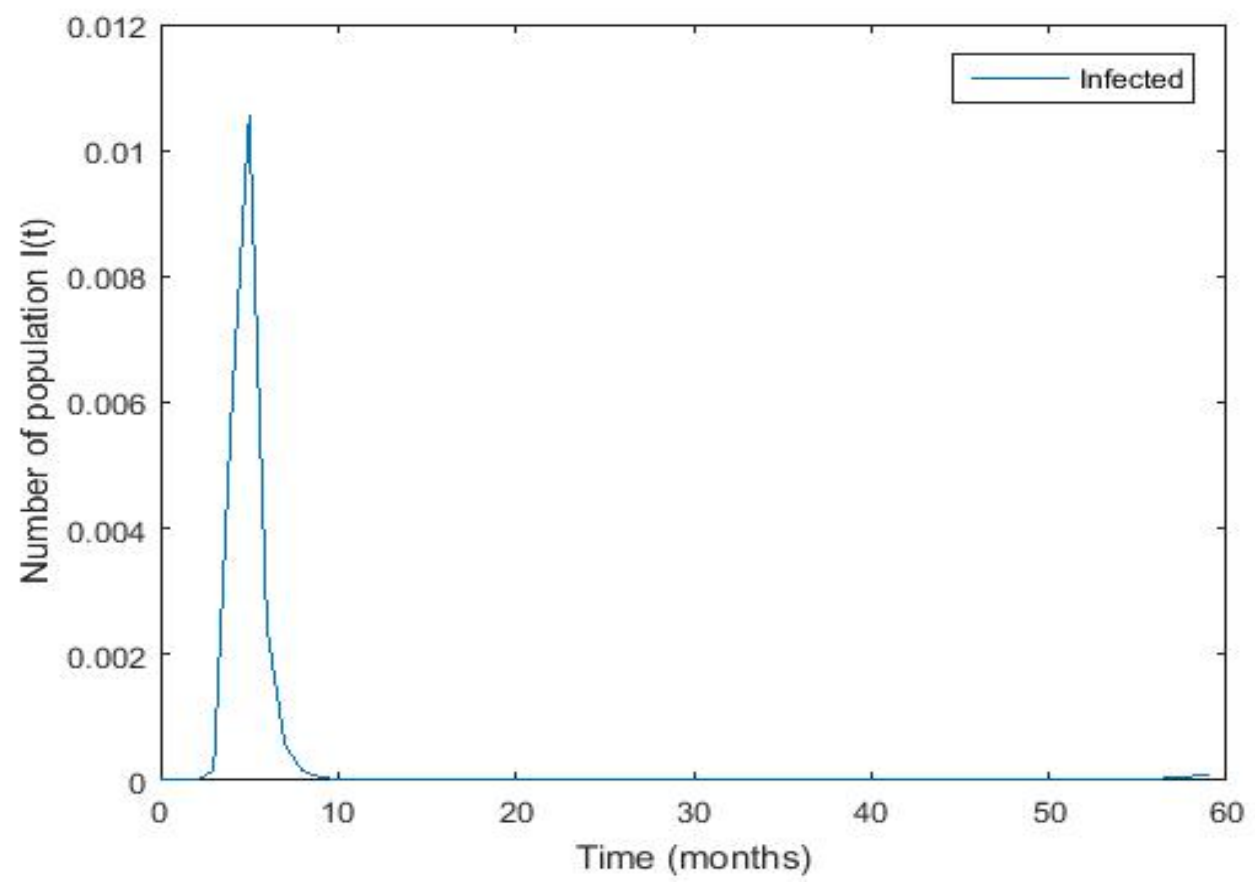

Figure 3.7: The simulation of the SIWDR model using initial values in table 3.3. The dynamic of the number of infected individuals is shown in this figure.

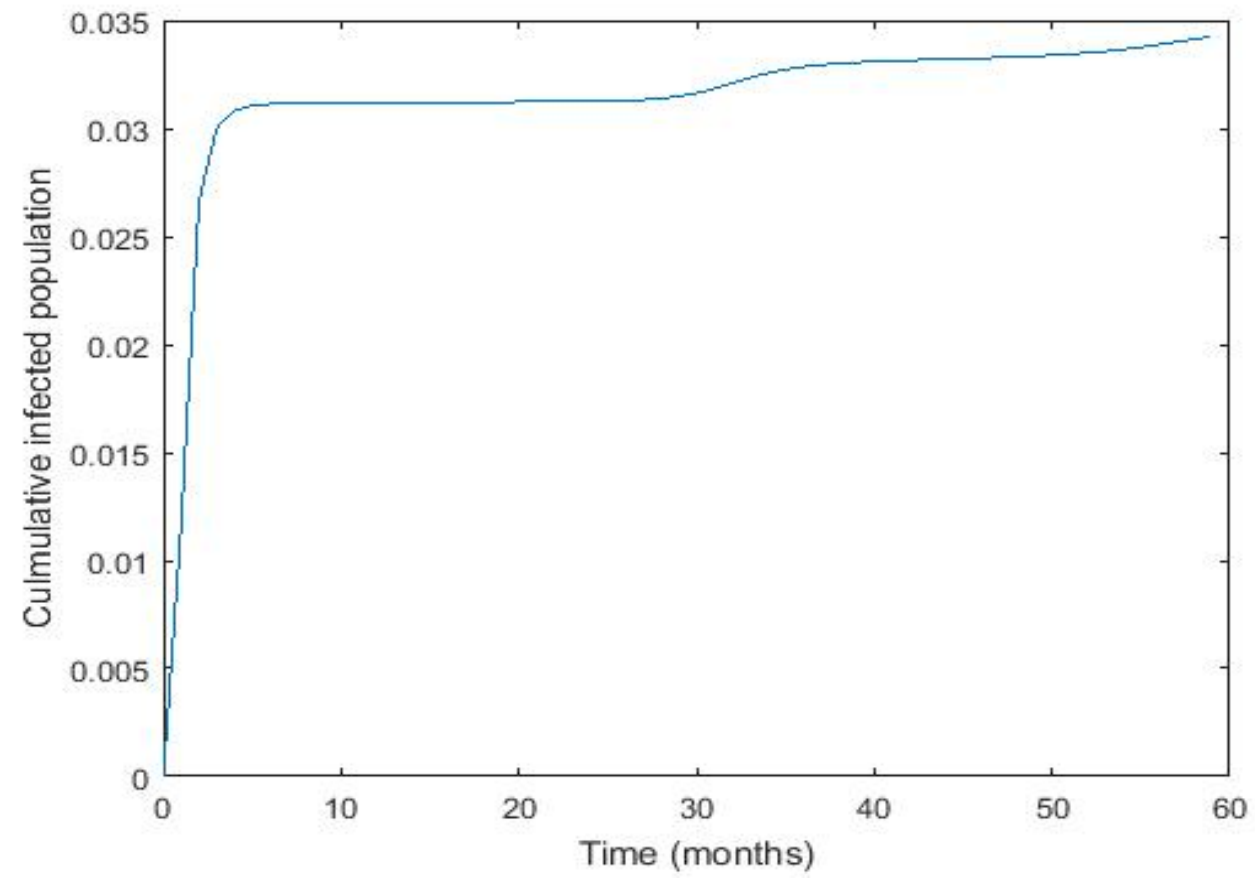

Figure 3.8: The simulation of the SIWDR model using initial values in table 3.3. The culmulative infected population is shown in this figure. 
It is interesting to note the dynamic behavior of compartment $I$. When I change some parameters* from the table 3.3, I see the so-called damping behavior. The peak that shows the number of infected population decreases over time; however, it occurs again, with a smaller peak height (Figure 3.9 below). Additionally, by varying other subsets of parameters, there could be more than 2 peaks. These trends are very similar to that of the actual number of infected population overtime. Hence, the SIWDR model is quite useful in predicting the number of infected population.

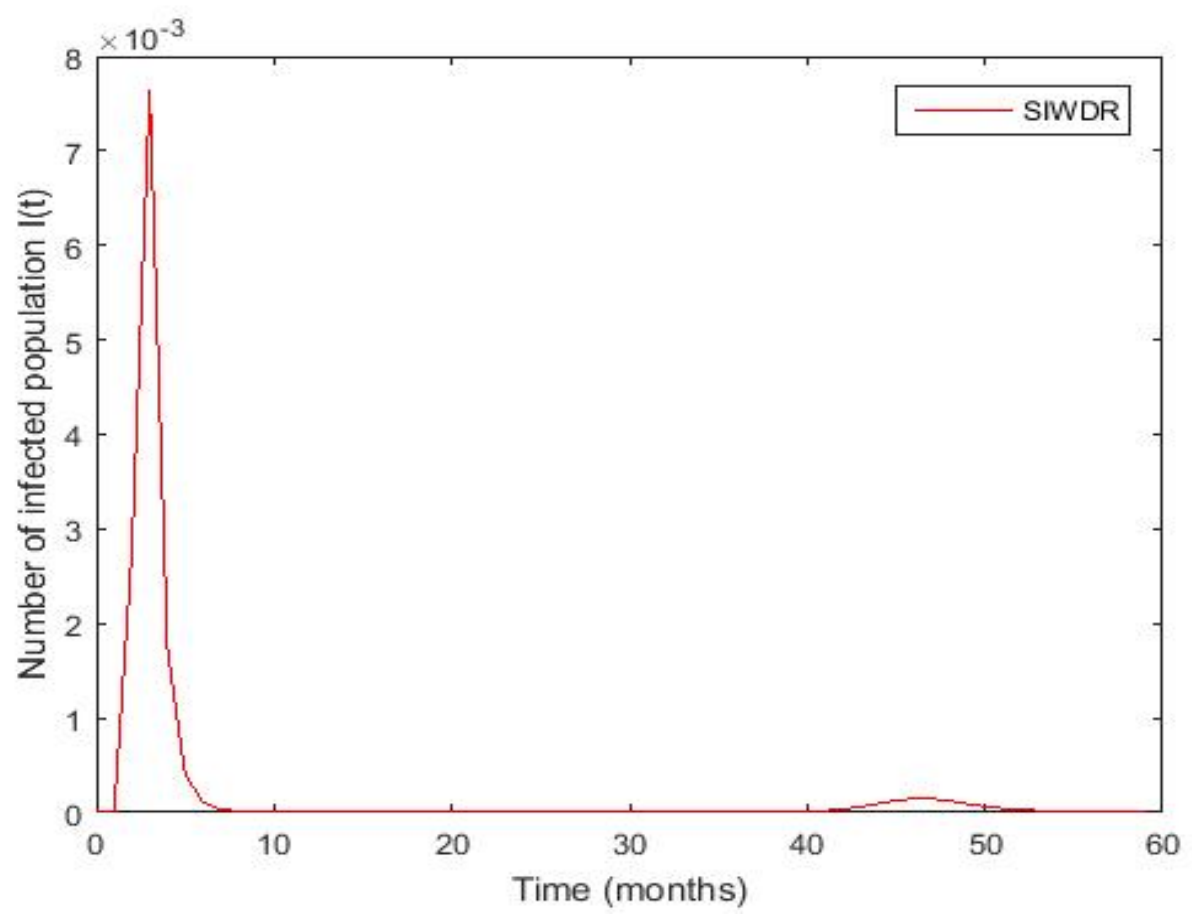

Figure 3.9: The number of infected population generated from the SIWDR model *(Note: the values of each parameter that is varied from the table 3.3 are as follows: $\beta_{I}=3, \beta_{W}=20, \beta_{D}=30, \alpha_{1}=300$, and $\alpha_{2}=50$ )

The dynamic behavior of the compartment $I$ is further observed when the value of $r_{1}, r_{2}$, and $r_{3}$ are varied. All other parameters are also tested with different values within their plausible ranges shown in the table 3.3 (See appendix B).

The basic reproduction number, $R_{0}$, is computed, and the number of infected population at the end of time horizon $(t=60)$ is observed. According to the known $R_{0}$, the stability of SIWDR model is determined. If $R_{0}<1$, then the steady-state 
is equal to 0 . If $R_{0}>1$, then the steady-state is not equal to 0 . I denote that $\varepsilon_{0}$ representing the steady-state is equal to 0 (the disease-free equilibrium), and I denote that $\varepsilon_{1}$ representing the steady-state is not equal to 0 (the endemic equilibrium). The table $3.4,3.5$, and 3.6 shows $R_{0}$ and the steady-state, with different range of $r_{1}, r_{2}$, and $r_{3}$. Please refer to chapter 4 for fundamental properties of the SIWDR model.

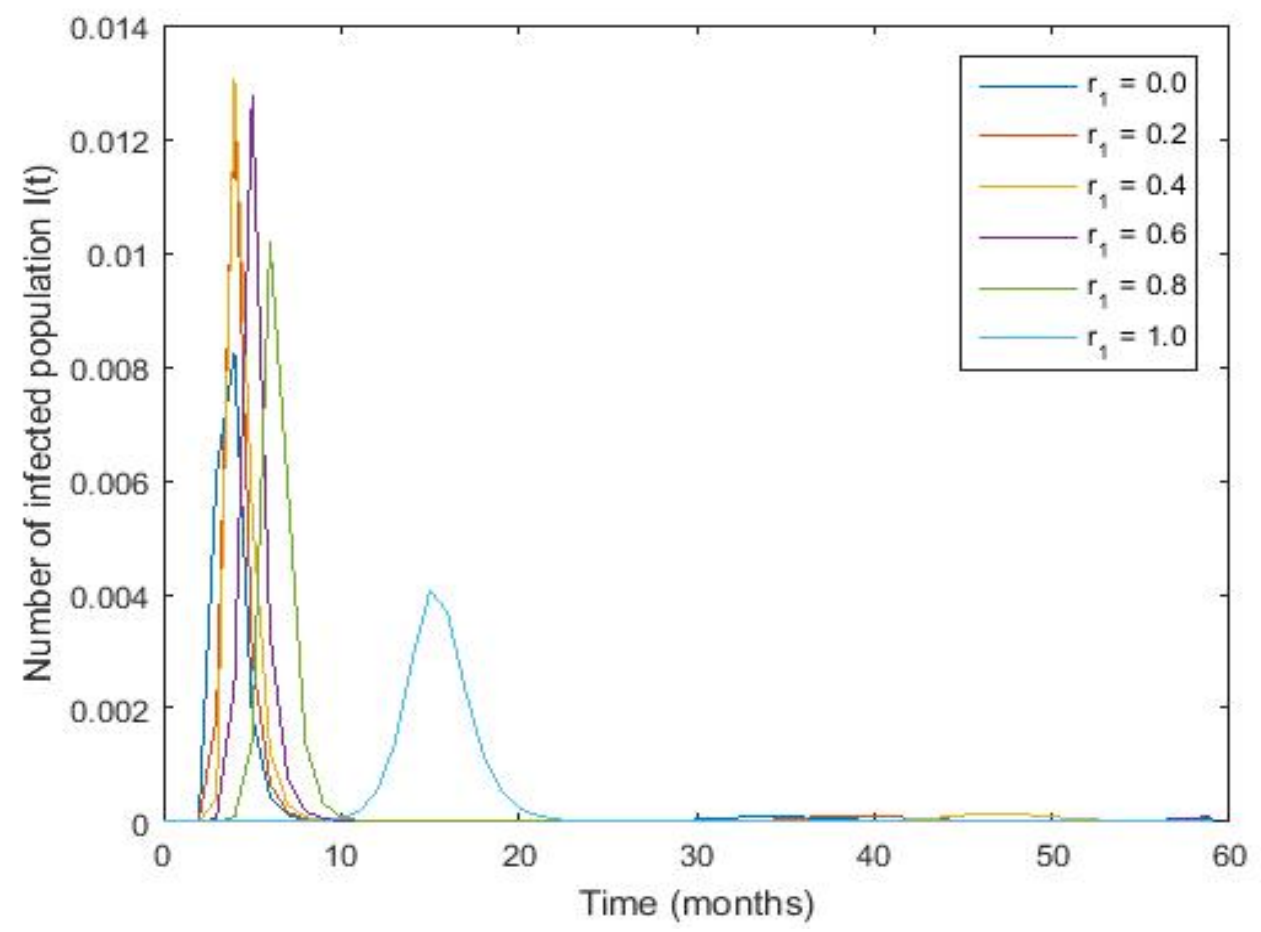

Figure 3.10: Plot shows the number of infected people with various values of parameter, $r_{1}$.

Table 3.4: Effect of $r_{1}$ on $R_{0}$ and $I$ at $t=60$.

\begin{tabular}{|c|c|c|c|}
\hline$r_{1}$ & $R_{0}$ & $I$ & Stable equilibrium \\
\hline 0.0 & 5.7952 & 0.0017 & $\varepsilon_{1}$ \\
\hline 0.2 & 4.6423 & 0.0006 & $\varepsilon_{1}$ \\
\hline 0.4 & 3.4895 & 0.0004 & $\varepsilon_{1}$ \\
\hline 0.6 & 2.3367 & 0.0011 & $\varepsilon_{1}$ \\
\hline 0.8 & 1.1839 & 0.0000 & $\varepsilon_{1}$ \\
\hline 1.0 & 0.0311 & 0.0098 & $\varepsilon_{0}$ \\
\hline
\end{tabular}




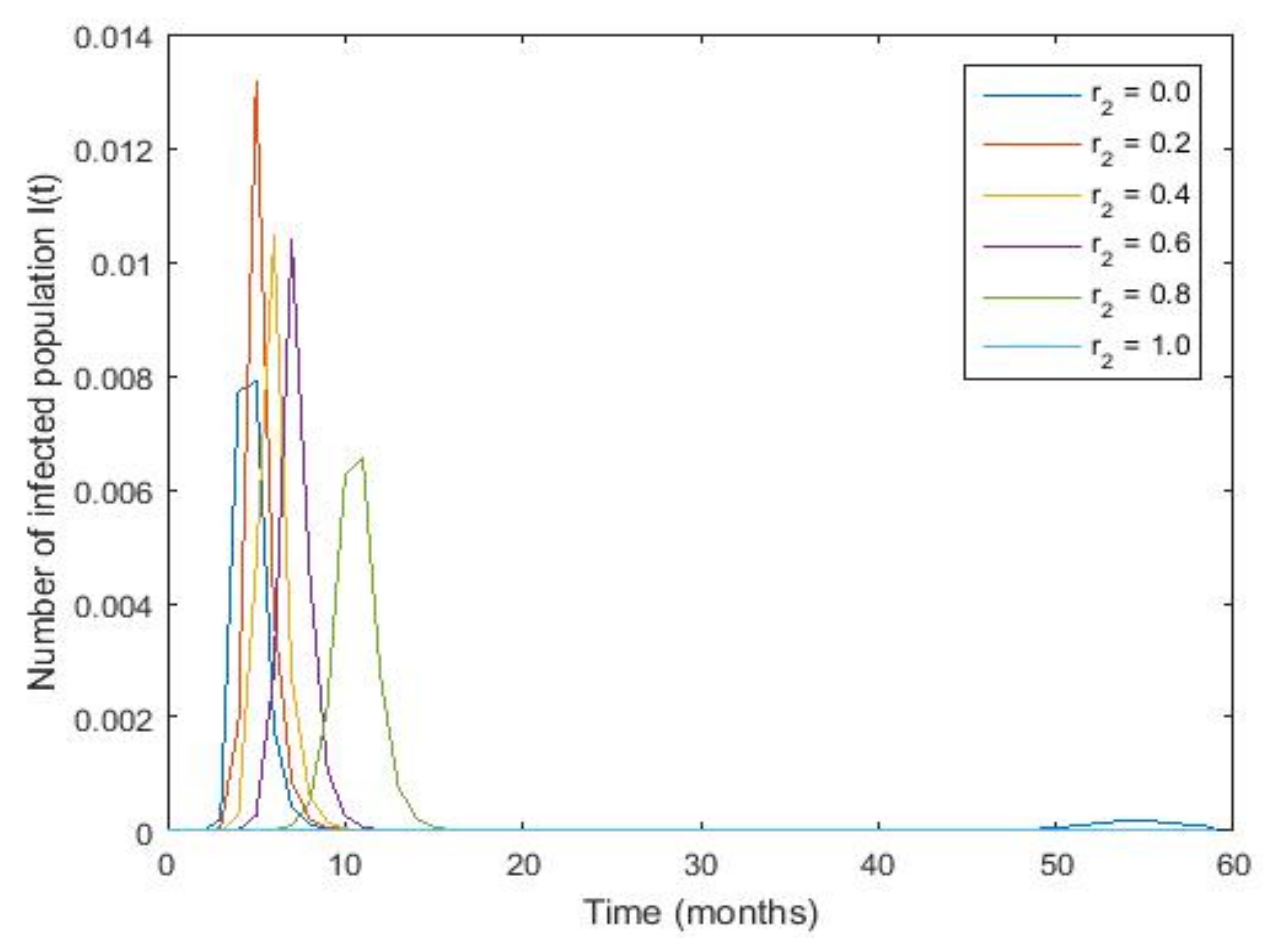

Figure 3.11: Plot shows the number of infected people with various values of parameter, $r_{2}$.

Table 3.5: Effect of $r_{2}$ on $R_{0}$ and $I$ at $t=60$.

\begin{tabular}{|c|c|c|c|}
\hline$r_{2}$ & $R_{0}$ & $I$ & Stable equilibrium \\
\hline 0.0 & 2.7192 & 0.0054 & $\varepsilon_{1}$ \\
\hline 0.2 & 2.1999 & 0.0003 & $\varepsilon_{1}$ \\
\hline 0.4 & 1.6806 & 0.0000 & $\varepsilon_{1}$ \\
\hline 0.6 & 1.1613 & 0.0000 & $\varepsilon_{1}$ \\
\hline 0.8 & 0.6420 & 0.0000 & $\varepsilon_{0}$ \\
\hline 1.0 & 0.1227 & 0.0000 & $\varepsilon_{0}$ \\
\hline
\end{tabular}

Once the $r_{1}, r_{2}$, and $r_{3}$ are obtained from the IWSS model $\left(G_{i j t}\right)$ (See the chapter 2 for more details), I increase the value of $r_{1}, r_{2}$, and $r_{3}$ as a result of capital investment. Increasing $r_{1}, r_{2}$, and $r_{3}$ helps to reduce cases of cholera infection seen in the figure 3.10, 3.11, and 3.12. In chapter 5, I use optimal control theory to find investment strategies for improving DWS, WST, and MSW services $\left(r_{1}, r_{2}\right.$, and $\left.r_{3}\right)$. This is to 
optimize the budget allocation across the three WatSan services over the planning horizon.

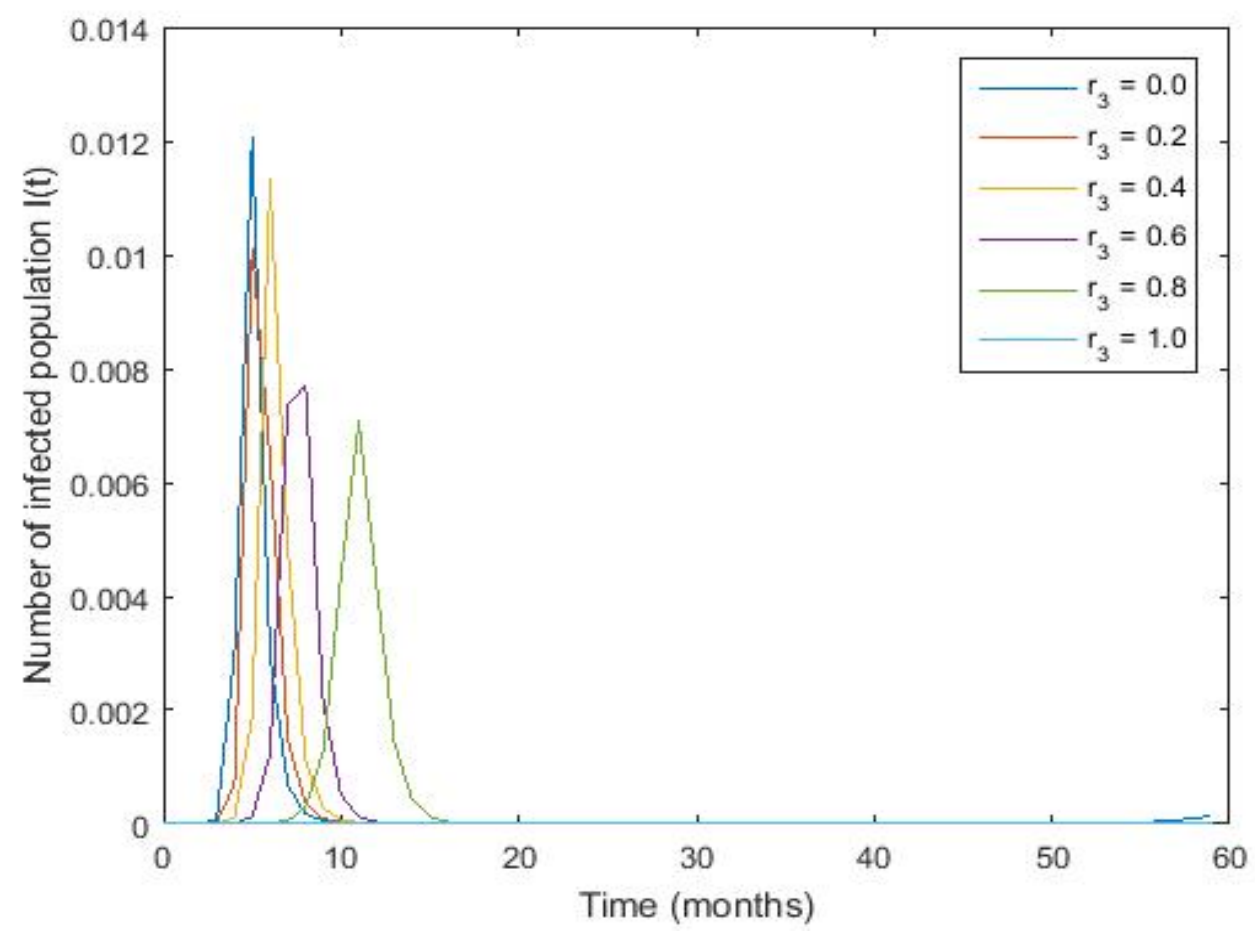

Figure 3.12: Plot shows the number of infected people with various values of parameter, $r_{3}$.

Table 3.6: Effect of $r_{3}$ on $R_{0}$ and $I$ at $t=60$.

\begin{tabular}{|c|c|c|c|}
\hline$r_{3}$ & $R_{0}$ & $I$ & Stable equilibrium \\
\hline 0.0 & 2.3648 & 0.0017 & $\varepsilon_{1}$ \\
\hline 0.2 & 1.9051 & 0.0000 & $\varepsilon_{1}$ \\
\hline 0.4 & 1.4454 & 0.0000 & $\varepsilon_{1}$ \\
\hline 0.6 & 0.9857 & 0.0000 & $\varepsilon_{0}$ \\
\hline 0.8 & 0.5259 & 0.0000 & $\varepsilon_{0}$ \\
\hline 1.0 & 0.0662 & 0.0000 & $\varepsilon_{0}$ \\
\hline
\end{tabular}




\subsection{WatSan's service impact}

Next, I compare the impact of each respective sanitation service on the incidence of cholera. Unit cost of each WatSan service is also specified as needed. Unit cost of WatSan services vary based on many factors such as types of technology, location, size of communities, and so on. However, I assume that an appropriate technology is house connection for the DWS service $(\$ 118)$ and simple pit latrine for WST service (\$43). The cost of DWS and WST service per capita is the average of the minimum $(\mathrm{DWS}=\$ 92, \mathrm{WST}=\$ 26)$ and maximum $(\mathrm{DWS}=\$ 144, \mathrm{WST}=\$ 60)$ cost for house connection and simple pit latrine per capita from literature [67] [9] . Regarding MSW service, I use the average cost of $\$ 32$ to calculate the impacts.

Table 3.7: Cost of WatSan services in LIC. Table includes WatSan services, cost per capita, ranges of cost per capita, technology, and source of the data.

\begin{tabular}{|l|l|l|l|}
\hline Service & Cost $(\$)$ & Technology & Source \\
\hline DWS & $92-144$ & House connection & WHO/UNICEF (2000) [67], Cairncross (2006) [9] \\
\hline DWS & $31-64$ & Stand post & WHO/UNICEF (2000) [67], Cairncross (2006) [9] \\
\hline DWS & $23-55$ & Borehole & WHO/UNICEF (2000) [67], Cairncross (2006) [9] \\
\hline DWS & $21-48$ & Dug well & WHO/UNICEF (2000) [67], Cairncross (2006) [9] \\
\hline DWS & $34-49$ & Rain Water & WHO/UNICEF (2000) [67], Cairncross (2006) [9] \\
\hline WST & $120-160$ & Sewer connection & WHO/UNICEF (2000) [67], Cairncross (2006) [9] \\
\hline WST & $52-112$ & Small bore sewer & WHO/UNICEF (2000) [67], Cairncross (2006) [9] \\
\hline WST & $104-160$ & Septic tank & WHO/UNICEF (2000) [67], Cairncross (2006) [9] \\
\hline WST & $50-91$ & Pour-flush & WHO/UNICEF (2000) [67], Cairncross (2006) [9] \\
\hline WST & $50-57$ & Ventilation-improved latrine & WHO/UNICEF $(2000)[67]$, Cairncross (2006) [9] \\
\hline WST & $26-60$ & Simple pit latrine & WHO/UNICEF (2000) [67], Cairncross (2006) [9] \\
\hline MSW & $16-100$ & not specified & Cointreau (2006) [21] \\
\hline
\end{tabular}

Finally, I compare cases of reduction in each service. Interestingly, figure 3.13 shows that WST and MSW services have higher rate of reduction of EDD than that of DWS service. WatSan managers would invest on WST and MSW services over the DWS service. However, this result occurs as a result of taking into account the high cost of technology within the DWS service. If low-cost technologies are used in the 
DWS service, the rate of reduction of EDD will turn out differently (See Table 3.7). That is to say, the rate of reduction of EDD depends on technologies used.

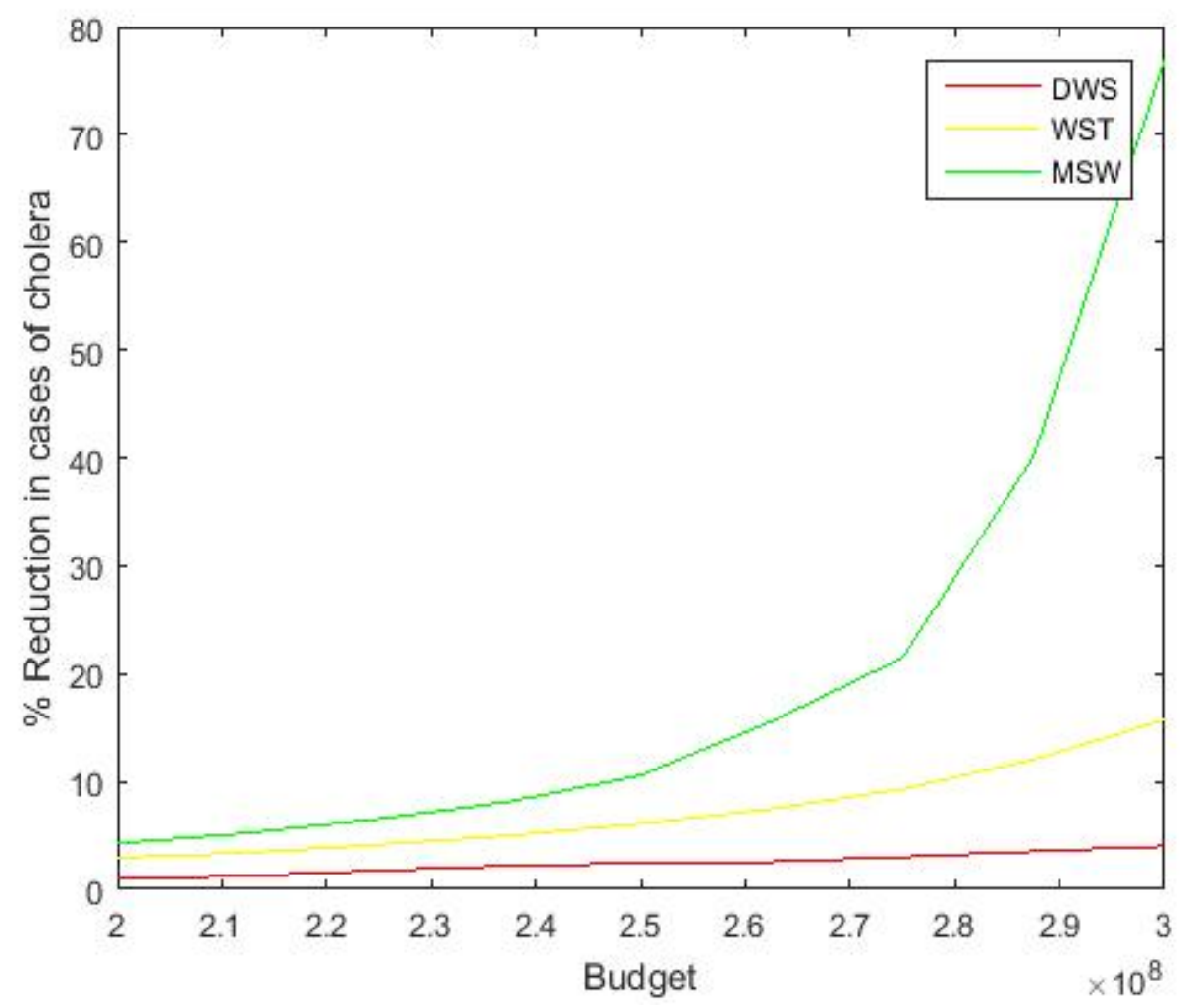

Figure 3.13: Comparison of WatSan serivce investment.

If data on the parameters of a particular disease and data on unit costs of WatSan technologies are available and reliable, I can improve the accuracy of this model so that it could be used for making practical WatSan budget allocation decisions. From the analyses/assumptions that I made in this chapter, I now have the system model - the model that links together the state variables of IWSS and the incidence of enteric diarrheal disease (EDD). I can later use this SIWDR model for simulation and optimization algorithms. 


\subsection{Summary of SIWDR model}

I propose a nonlinear differential equations model called SIWDR (Susceptible-InfectedWater-Dumpsite-Recovered), which relates the water and sanitation (WatSan) deficit to the total incidents of enteric diarrheal disease (EDD) in a specific service region. The SIWDR model is extended from the SIWR (Susceptible-Infected-Water-Recovered)(Tien and Earn, 2010) [69], which is used to analyze the infectious diseases such as cholera. The SIWDR is built by adding the compartment D (Dumpsite) into the SIWR model. D compartment is added to complete WatSan services. Also, dumpsite is an important transmission media of water-borne diseases. I use data from the cholera outbreak in Haiti from 2010 to 2015 to test the SIWDR model. I also compare cases of disease reduction in DWS, WST, and MSW. As a result, I can allocate the budget across these three services in such a way that I minimize the incidence of EDD. 


\section{Chapter 4}

\section{Fundamental Properties of SIWDR Model}

This chapter investigates many fundamental properties of the dynamics of the water and sanitation (WatSan) deficit and its public health impact in a community. These dynamics are captured in the SIWDR model. Both disease-free equilibrium and endemic equilibrium of SIWDR are observed. In addition, the stability of both equilibria is examined.

\subsection{Feasible region of the equilibria of the model}

All parameters and state variables are assumed to be non-negative because the system model (3.4) represents human and pathogen populations. Also, $r_{1}, r_{2}, r_{3}$ are fraction of DWS, WST, MSW coverage respectively, $0 \leq r_{1} \leq 1,0 \leq r_{2} \leq 1,0 \leq r_{3} \leq 1$. Further, it remains to verified that all solutions with non-negative intitial conditions are bounded and non-negative. 
Adding the first, second, and fifth equation of (3.4) and $n=s+i+r$.

$$
\begin{aligned}
\dot{n} & =\dot{s}+\dot{i}+\dot{r} \\
& =\Lambda-\mu s-\mu i-\mu r \\
& =\Lambda-\mu(s-i-r) \\
& =\Lambda-\mu n
\end{aligned}
$$

Using an integrating factor, $e^{\mu t}$, I have

$n(t)=\frac{\Lambda}{\mu}+C e^{-\mu t} \Rightarrow \lim _{t \rightarrow \infty} n(t)=\frac{\Lambda}{\mu} \Rightarrow \lim _{t \rightarrow \infty} \sup (n(t)) \leq \frac{\Lambda}{\mu}$

Thus, $s \leq \frac{\Lambda}{\mu}, i \leq \frac{\Lambda}{\mu}$ and $r \leq \frac{\Lambda}{\mu}$

Consider,

$$
\begin{aligned}
\dot{d} & =\xi_{D}\left(1-r_{2}\right) i-\xi_{D} d \\
& \leq \xi_{D}\left(1-r_{2}\right) \frac{\Lambda}{\mu}-\xi_{D} d
\end{aligned}
$$

$\Rightarrow \lim _{t \rightarrow \infty} \sup (d(t)) \leq \frac{\Lambda\left(1-r_{2}\right)}{\mu}$

Thus, $d \leq \frac{\Lambda\left(1-r_{2}\right)}{\mu}$

Next, consider

$$
\begin{gathered}
w^{\prime}=\xi_{W}\left(1-r_{2}\right) i+\frac{\left(1-r_{3}\right) \alpha_{2} \xi_{W} \psi}{\alpha_{1} \xi_{D}} d-\xi_{W} w \\
\leq \xi_{W}\left(1-r_{2}\right) \frac{\Lambda}{\mu}+\frac{\left(1-r_{3}\right) \alpha_{2} \xi_{W} \psi}{\alpha_{1} \xi_{D}}\left(\frac{\Lambda\left(1-r_{2}\right)}{\mu}\right)-\xi_{W} w \\
=\frac{\Lambda \xi_{W}\left(1-r_{2}\right)}{\mu}\left(1+\frac{\left(1-r_{3}\right) \alpha_{2} \psi}{\alpha_{1} \xi_{D}}\right)-\xi_{W} w \\
\Rightarrow \lim _{t \rightarrow \infty} \sup (w(t)) \leq \frac{\Lambda\left(1-r_{2}\right)}{\mu}\left(1+\frac{\left(1-r_{3}\right) \alpha_{2} \psi}{\alpha_{1} \xi_{D}}\right) \\
\text { Thus, } w \leq \frac{\Lambda\left(1-r_{2}\right)}{\mu}\left(1+\frac{\left(1-r_{3}\right) \alpha_{2} \psi}{\alpha_{1} \xi_{D}}\right)
\end{gathered}
$$

Hence, all feasible solution of system model (3.4) are positive and ultimately enter the 
invariant attracting region.

$$
\Omega=\left\{(s, i, r, w, d): 0 \leq s+i+r \leq \frac{\Lambda}{\mu}, w \leq \frac{\Lambda\left(1-r_{2}\right)}{\mu}\left(1+\frac{\left(1-r_{3}\right) \alpha_{2} \psi}{\alpha_{1} \xi_{D}}\right), d \leq \frac{\Lambda\left(1-r_{2}\right)}{\mu}\right\}
$$

Thus, existence, uniqueness, and continuation results for system model (3.4) hold in $\Omega$ and all solutions starting in $\Omega$ remain in $\Omega$ for all $t \geq 0$. The system model (3.4) is mathematically and epidemiologically well-posed and it is sufficient to consider the dynamics of the flow generated by the system model (3.4) in $\Omega$.

There are two equilibria in the system model (3.4). The first equilibrium is called disease-free equilibrium, where there is no disease in the system model. The second equilibrium is endemic equilibrium, this equilibrium is attained while there is disease in the system model. The basic reproduction number, $R_{0}$, is defined as the expected number of cases that one infected individual can produce by infecting other susceptible individuals during its infective period [74]. $R_{0}$ at both equilibrium will be calculated because $R_{0}$ is the most important quantity in mathematical epidemiology. This quantity can indicate whether disease will break out or not. $R_{0}$ is calculated by using the next generation matrix approach [75]. In addition, this following section describes the method to linearize the system model (3.4) and process to attain local and global statbility of disease-free equilibrium from van den Driessche and Watmough (2008) [74]. The outline (e.g., lemmas and theorems) of this chapter is adapted from Sharomi and Gumel (2008)'s paper [65].

\subsection{Stability of disease-free equilibrium (DFE)}

\subsubsection{Local stability}

First, let's analyze $R_{0}$ at the disease-free equilibrium. Since compartment $i, w$, and $d$ have disease, compartment $i, w$, and $d$ are assumed to be zero. Hence, the system 
model (3.4) has disease-free equilibrium at

$$
\varepsilon_{0}:\left(s^{*}, i^{*}, w^{*}, d^{*}, r^{*}\right)=\left(\frac{\Lambda}{\mu}, 0,0,0,0\right)
$$

Next, the next generation method is used to compute $R_{0}$. I use the van den Driessche and Watmough (2008)'s next generation method, which is shown below [74]. This method is applied to the SIWDR model. They assume there are $n$ disease compartments and $m$ non-disease compartment, and let $x \in R^{n}$ and $y \in R^{m}$ be subpopulations in each of these compartments. Now let $F_{i}^{0}$ be the rate of secondary infections increase the $i^{\text {th }}$ disease compartment and let $V_{i}^{0}$ be the rate disease progression, death, and recovery reduce the $i^{\text {th }}$ compartment. Then, the compartmental model can be written as follow.

$$
\begin{gathered}
\dot{x}_{i}=F_{i}^{0}(x, y)-V_{i}^{0}, \quad i=1, \ldots, n \\
\dot{y}_{j}=g_{j}{ }^{0}(x, y), \quad j=1, \ldots, m
\end{gathered}
$$

The next generation matrix is written at the disease free equilibrium as $F V^{-1}$. The entry $(i, j)$ of matrix $F$ is the rate at which infected individual in compartment $j$ produce new infections in compartment $i$, and the $(i, j)$ entry of matrix $V^{-1}$ is the expected time of stay in compartment $i$ starting from compartment $j$. Now,

$$
R_{0}=\rho\left(F V^{-1}\right)
$$

is calculated, where $\rho$ is defined as sprectal radius of matrix $F V^{-1}$ (the largest eigenvalue of $F V^{-1}$ ). For the system model (3.4), first I write the system model (3.4) in the form of disease and nondisease compartments and then linearized (4.2). I then 
write the system model with compartment $i, w, d$ as

$$
\dot{x}=(F-V)^{T} x
$$

where $F=\frac{\partial F_{i}{ }^{0}\left(0, y_{0}\right)}{\partial x_{j}} \quad$ and $\quad V=\frac{\partial V_{i}{ }^{0}\left(0, y_{0}\right)}{\partial x_{j}}$.

There are assumptions to ensure the existence of this equilibrium and ensure the model is well posed on $F_{i}^{0}\left(x_{0}, y_{0}\right)$ and $V_{i}^{0}\left(x_{0}, y_{0}\right)$; these assumptions can be viewed on van den Driessche and Watmough (2008) [74] (see also section A.1 of Appendix A). Now, the stability of the system model (3.4) at the disease-free equilibrium is completely determined by the linear stability of $(F-V)$. Then, I have $F^{0}$ and $V^{0}$ as follow

$$
\begin{gathered}
F^{0}=\left[\begin{array}{c}
\left(1-r_{1}\right) \beta_{W} s w+\left(1-r_{3}\right) \beta_{D} s d+\beta_{I} s i \\
0 \\
0
\end{array}\right] \\
V^{0}=\left[\begin{array}{c}
\gamma i+\mu i \\
-\xi_{W}\left(1-r_{2}\right) i-\frac{\left(1-r_{3}\right) \alpha_{2} \xi_{W} \psi}{\alpha_{1} \xi_{D}} d+\xi_{W} w \\
-\xi_{D}\left(1-r_{2}\right) i+\xi_{D} d
\end{array}\right]
\end{gathered}
$$

Now, I can compute $F$ and $V$ as follow

$$
\begin{gathered}
F=\left[\begin{array}{ccc}
\beta_{I}\left(\frac{\Lambda}{\mu}\right) & \left(1-r_{1}\right) \beta_{W}\left(\frac{\Lambda}{\mu}\right) & \left(1-r_{3}\right) \beta_{D}\left(\frac{\Lambda}{\mu}\right) \\
0 & 0 & 0 \\
0 & 0 & 0
\end{array}\right] \\
V=\left[\begin{array}{ccc}
\gamma+\mu & 0 & 0 \\
-\xi_{W}\left(1-r_{2}\right) & \xi_{W} & -\frac{\left(1-r_{3}\right) \alpha_{2} \xi_{W} \psi}{\alpha_{1} \xi_{D}} \\
-\xi_{D}\left(1-r_{2}\right) & 0 & \xi_{D}
\end{array}\right]
\end{gathered}
$$


Then, I compute $V^{-1}$

$$
V^{-1}=\left[\begin{array}{ccc}
\frac{1}{\gamma+\mu} & 0 & 0 \\
\frac{\left(1-r_{2}\right)\left(\alpha_{2} \psi+\alpha_{1} \xi_{D}-\alpha_{2} \psi r_{3}\right)}{\alpha_{1} \xi_{D}(\gamma+\mu)} & \frac{1}{\xi_{W}} & \frac{\left(1-r_{3}\right) \alpha_{2} \psi}{\alpha_{1} \xi_{D}^{2}} \\
\frac{\left(1-r_{2}\right)}{\gamma+\mu} & 0 & \frac{1}{\xi_{D}}
\end{array}\right]
$$

The basic reproduction number, $R_{0}$, is then determined by the spectral radius of the matrix $F V^{-1}$

$$
\begin{aligned}
& R_{0}=\rho\left(F V^{-1}\right) \\
& =\frac{\Lambda \beta_{I}+\Lambda \beta_{D}\left(1-r_{2}\right)\left(1-r_{3}\right)}{\mu(\gamma+\mu)}+\frac{\Lambda \beta_{W}\left(1-r_{1}\right)\left(1-r_{2}\right)\left(\alpha_{2} \psi+\alpha_{1} \xi_{D}-\alpha_{2} \psi r_{3}\right)}{\mu(\gamma+\mu) \alpha_{1} \xi_{D}} \\
& =\frac{\Lambda\left(\beta_{I} \alpha_{1} \xi_{D}+\beta_{W}\left(1-r_{1}\right)\left(1-r_{2}\right) \alpha_{1} \xi_{D}+\beta_{W}\left(1-r_{1}\right)\left(1-r_{2}\right)\left(1-r_{3}\right) \alpha_{2} \psi+\beta_{D}\left(1-r_{2}\right)\left(1-r_{3}\right) \alpha_{1} \xi_{D}\right)}{\mu(\gamma+\mu) \alpha_{1} \xi_{D}}
\end{aligned}
$$

From Theorem 1 in Van den Driessche and Watmough (2002) [74] (see also Theorem 5 of Appendix A.2), the following result is established.

Lemma 1. For the system (3.4), the DFE, $\varepsilon_{0}$, is locally-asymptotically stable (LAS)

$$
\text { if } R_{0}<1 \text {, and unstable if } R_{0}>1 \text {. }
$$

The local stability result in Lemma 1 implies that for $R_{0}<1$, the total number of infected people in the population can be reduced to zero if the initial sizes of the sub-populations of the model are in the basin of attraction of $\varepsilon_{0}$. It is important to show that DFE is globally-asymtotically stable (GAS) because the control of the number of infected population at the steady-state is not effective when the control depends on the initial sizes of the sub-populations of the model. This following section shows that DFE is GAS. 


\subsubsection{Global stability of DFE}

I prove the following:

Theorem 1. The DFE is globally-asymptotically stable (GAS) in $\Omega$ if $R_{0}<1$.

Proof. For the global stability at the disease-free equilibrium, I need to rewrite equations (4.2) and (4.3) again in form

$$
\begin{gathered}
\dot{x}=-A x-f(x, y), \\
\dot{y}=g(x, y) .
\end{gathered}
$$

By Theorem 2 [74] (see also Theorem 6 of Appendix A.3), two conditions need to be checked.

(c1) $A$ is a non-singular $M$-matrix

(c2) $f \geq 0$

If these two conditions hold, then the disease-free equilibrium of (3.4) is globally asymptotically stable. Choose $A=V-F$ and write the system model (3.4) in the form (4.6) and (4.7) as follows

$$
\dot{x}=-(V-F) x-\left(\begin{array}{c}
\beta_{I}\left(\frac{\Lambda}{\mu}-s\right) i+\left(1-r_{1}\right) \beta_{W}\left(\frac{\Lambda}{\mu}-s\right) w+\left(1-r_{3}\right) \beta_{D}\left(\frac{\Lambda}{\mu}-s\right) d \\
0 \\
0
\end{array}\right)
$$




$$
\begin{aligned}
& \dot{s}=\Lambda-\left(1-r_{1}\right) \beta_{W} s w-\left(1-r_{3}\right) \beta_{D} s d-\beta_{I} s i-\mu s \\
& \dot{r}=\gamma i-\mu r
\end{aligned}
$$

It is clear that matrix $(V-F)$ is a non-singular $M$-matrix since the off diagonal elements of $(V-F)$ are negative. Hence, to show that the disease-free equilibrium of (3.4) is globally-asymptotically stable (GAS) for $R_{0}<1$, it left to show that $f \geq 0$.

Since $\frac{\Lambda}{\mu}>s$, see the proof in section 4.1 when I show that $s \leq \frac{\Lambda}{\mu}$. Thus, $f \geq 0$. I can conclude that the disease-free equilibrium of (3.4) is GAS.

\subsection{Endemic equilibrium (EE)}

\subsubsection{Existence of endemic equilibrium}

The existence of endemic equilibrium is explored as follows. The state variables of the model (3.4), can be expressed at an arbitary EE, donoted by

$$
\varepsilon_{1}=\left(s^{*}, i^{*}, w^{*}, d^{*}, r^{*}\right)
$$

The EE is obtained by setting the right-hand sides of equation (3.4) to zero. The endemic equilibrium point is an implitcit solution. Hence, I need to write the solution in terms of one variable; I select to write the solution in term of $i^{*}$ as follow. Let the last equation of the system model (3.4) equal to 0 .

$$
r^{*}=\frac{\gamma}{\mu} i^{*}
$$

Let $\dot{d}$ in the system model (3.4) equal to 0 .

$$
d^{*}=\left(1-r_{2}\right) i^{*}
$$


Let $\dot{w}$ in the system model (3.4) equal to 0 , replace (4.12) into $d^{*}$.

Let $k=\left(1-r_{2}\right)\left(1+\frac{\left(1-r_{3}\right) \alpha_{2} \psi}{\alpha_{1} \xi_{D}}\right)$

$$
\begin{aligned}
w^{*} & =\left(1-r_{2}\right) i^{*}+\frac{\left(1-r_{3}\right) \alpha_{2} \psi}{\alpha_{1} \xi_{D}} d^{*} \\
& =\left(1-r_{2}\right) i^{*}+\frac{\left(1-r_{3}\right) \alpha_{2} \psi}{\alpha_{1} \xi_{D}}\left(1-r_{2}\right) i^{*} \\
& =\left(1-r_{2}\right)\left(1+\frac{\left(1-r_{3}\right) \alpha_{2} \psi}{\alpha_{1} \xi_{D}}\right) i^{*} \\
& =k i^{*}
\end{aligned}
$$

Let $\dot{s}$ in the system model (3.4) equals to 0 , replace (4.12) into $d^{*}$, and (4.13) into $w^{*}$.

$$
\begin{aligned}
s^{*} & =\frac{\Lambda}{\left(1-r_{1}\right) \beta_{W} w^{*}+\left(1-r_{3}\right) \beta_{D} d^{*}+\beta_{I} i^{*}+\mu} \\
& =\frac{\Lambda}{\left(1-r_{1}\right) \beta_{W} k i^{*}+\left(1-r_{2}\right)\left(1-r_{3}\right) \beta_{D} i^{*}+\beta_{I} i^{*}+\mu}
\end{aligned}
$$

Thus, $\left(s^{*}, i^{*}, w^{*}, d^{*}, r^{*}\right)=\left(\frac{\Lambda}{\left(1-r_{1}\right) \beta_{W} k i^{*}+\left(1-r_{2}\right)\left(1-r_{3}\right) \beta_{D} i^{*}+\beta_{I} i^{*}+\mu}, i^{*}, k i^{*},\left(1-r_{2}\right) i^{*}, \frac{\gamma}{\mu} i^{*}\right)$

Next, an endemic equilibrium for the system model (3.4) when $R_{0}>1$ will be examimed. First, I will prove that the system model (3.4) has a unique endemic equilibrium.

Theorem 2. The system model (3.4) has a unique endemic equilibrium (EE), $\varepsilon_{1}$, if $R_{0}>1$, and no EE if $R_{0} \leq 1$.

Proof. Let $s^{*}, i^{*}, w^{*}, d^{*}, r^{*}$ be a constant solution of the system model (3.4). I can express $s^{*}, w^{*}, d^{*}, r^{*}$ in term of $i^{*}$ as shown previously. I substitute (4.11), (4.12), (4.13), (4.14) in the equation for $\dot{i}$ and set $\dot{i}=0$ given by 


$$
\begin{aligned}
& 0=\left(1-r_{1}\right) \beta_{W} s^{*} w^{*}+\left(1-r_{3}\right) \beta_{D} s^{*} d^{*}+\beta_{I} s^{*} i^{*}-\gamma i^{*}-\mu i^{*} \\
& 0=\left(1-r_{1}\right) \beta_{W} s^{*} k i^{*}+\left(1-r_{3}\right) \beta_{D} s^{*}\left(1-r_{2}\right) i^{*}+\beta_{I} s^{*} i^{*}-\gamma i^{*}-\mu i^{*} \\
& 0=i^{*}\left(\left(1-r_{1}\right) \beta_{W} s^{*} k+\left(1-r_{3}\right) \beta_{D} s^{*}\left(1-r_{2}\right)+\beta_{I} s^{*}-\gamma-\mu\right)
\end{aligned}
$$

I notice that $i^{*}=0$, is the point where I have the disease-free equilibrium. Thus, endemic equilibrium exists when

$$
0=\left(1-r_{1}\right) \beta_{W} s^{*} k+\left(1-r_{3}\right) \beta_{D} s^{*}\left(1-r_{2}\right)+\beta_{I} s^{*}-\gamma-\mu
$$

I rearrange the equation (4.15) above to involve $R_{0}$ as follow.

$$
0=s^{*}\left(\left(1-r_{1}\right) \beta_{W} k+\left(1-r_{3}\right) \beta_{D}\left(1-r_{2}\right)+\beta_{I}\right)-(\gamma+\mu)
$$

Replace (4.14) into $s^{*}$

$$
0=\frac{\Lambda}{\left(1-r_{1}\right) \beta_{W} k i^{*}+\left(1-r_{2}\right)\left(1-r_{3}\right) \beta_{D} i^{*}+\beta_{I} i^{*}+\mu}\left(\left(1-r_{1}\right) \beta_{W} k+\left(1-r_{3}\right) \beta_{D}\left(1-r_{2}\right)+\beta_{I}\right)-(\gamma+\mu)
$$

Let $A=\left(1-r_{1}\right) \beta_{W} k+\left(1-r_{2}\right)\left(1-r_{3}\right) \beta_{D}+\beta_{I}$. Then,

$$
\begin{array}{r}
(\gamma+\mu)\left(A i^{*}+\mu\right)=\Lambda\left(\left(1-r_{1}\right) \beta_{W} k+\left(1-r_{3}\right) \beta_{D}\left(1-r_{2}\right)+\beta_{I}\right) \\
i^{*}=\frac{\Lambda\left(\left(1-r_{1}\right) \beta_{W} k+\left(1-r_{3}\right) \beta_{D}\left(1-r_{2}\right)+\beta_{I}\right)-\mu(\gamma+\mu)}{(\gamma+\mu) A}
\end{array}
$$

Divide $\mu(\gamma+\mu)$ on the right hand side of the equation above on both numerator and denominator and then consider the numerator only. 
I have

$$
\begin{aligned}
& \frac{\Lambda\left(\left(1-r_{1}\right) \beta_{W} k+\left(1-r_{3}\right) \beta_{D}\left(1-r_{2}\right)+\beta_{I}\right)-\mu(\gamma+\mu)}{\mu(\gamma+\mu)}=\frac{\Lambda\left(\left(1-r_{1}\right) \beta_{W} k+\left(1-r_{3}\right) \beta_{D}\left(1-r_{2}\right)+\beta_{I}\right)}{\mu(\gamma+\mu)}-1 \\
& =\frac{\Lambda\left(\left(1-r_{1}\right) \beta_{W}\left(1-r_{2}\right)\left(1+\frac{\left(1-r_{3}\right) \alpha_{2} \psi}{\alpha_{1} \xi_{D}}\right)+\left(1-r_{3}\right) \beta_{D}\left(1-r_{2}\right)+\beta_{I}\right)}{\mu(\gamma+\mu)}-1 \\
& =\frac{\Lambda\left(\left(1-r_{1}\right) \beta_{W}\left(1-r_{2}\right)\left(\alpha_{1} \xi_{D}+\left(1-r_{3}\right) \alpha_{2} \psi\right)+\left(1-r_{3}\right) \beta_{D}\left(1-r_{2}\right) \alpha_{1} \xi_{D}+\beta_{I} \alpha_{1} \xi_{D}\right)}{\mu(\gamma+\mu) \alpha_{1} \xi_{D}}-1 \\
& =\frac{\Lambda\left(\beta_{I} \alpha_{1} \xi_{D}+\beta_{W}\left(1-r_{1}\right)\left(1-r_{2}\right) \alpha_{1} \xi_{D}+\beta_{W}\left(1-r_{1}\right)\left(1-r_{2}\right)\left(1-r_{3}\right) \alpha_{2} \psi+\beta_{D}\left(1-r_{2}\right)\left(1-r_{3}\right) \alpha_{1} \xi_{D}\right)}{\mu(\gamma+\mu) \alpha_{1} \xi_{D}}-1 \\
& =R_{0}-1
\end{aligned}
$$

Consider the denominator of (4.16),

$$
\frac{(\gamma+\mu) A}{\mu(\gamma+\mu)}=\frac{A}{\mu}
$$

Now assume $\frac{A}{\mu}=a_{0}$ and $R_{0}-1=a_{1}$. Thus, I have

$$
i^{*}=\frac{R_{0}-1}{A / \mu}=\frac{a_{1}}{a_{0}}
$$

I obtain

$$
a_{0} i^{*}-a_{1}=0
$$

Further, $a_{1}>0$ for $R_{0}>0$. Thus, the linear equation (4.17) has a unique positive solution when $R_{0}>1$ (i.e. $i^{*}>0$ if and only if $R_{0}>1$ ). It should noted that if $R_{0}<1$, then $a_{1}<0$. Hence, for $R_{0}<1$, the quantity $i^{*}<0$ (which is biologically 
meaningless). Consequently, the endemic equilibrium, $\varepsilon_{1}$, does not exist in this case. Furthermore, for $R_{0}=1$, the coefficient $a_{1}=0$; making $i^{*}=0$ (coresponding to DFE).

\subsubsection{Local stability of endemic equilibrium}

The local stability of the unique endemic equilibrium (EE) is explored. I prove the following:

Theorem 3. The unique EE of the model (3.4), is LAS if $R_{0}>1$ and is close to 1 .

Proof. Due to its high dimesionality, linearizing the model (3.4) around its unique EE is burdensome. Therefore, I consider to use the centre manifold theory [11]. To apply this method, I need to simplify and change variables in the model (3.4). Let $s=x_{1}, i=x_{2}, w=x_{3}, d=x_{4}$, and $r=x_{5}$, so that $n=x_{1}+x_{2}+x_{5}$. Further, by using vector notation $X=\left(x_{1}, x_{2}, x_{3}, x_{4}, x_{5}\right)^{T}$. The model (3.4) can be written in the form $\frac{d X}{d t}=\left(f_{1}, f_{2}, f_{3}, f_{4}, f_{5}\right)^{T}$, as follows:

$$
\begin{aligned}
& \frac{d x_{1}}{d t}=f_{1}=\Lambda-\left(1-r_{1}\right) \beta_{W} x_{1} x_{3}-\left(1-r_{3}\right) \beta_{D} x_{1} x_{4}-\beta_{I} x_{1} x_{2}-\mu x_{1} \\
& \frac{d x_{2}}{d t}=f_{2}=\left(1-r_{1}\right) \beta_{W} x_{1} x_{3}+\left(1-r_{3}\right) \beta_{D} x_{1} x_{4}+\beta_{I} x_{1} x_{2}-\gamma x_{2}-\mu x_{2} \\
& \frac{d x_{3}}{d t}=f_{3}=\xi_{W}\left(1-r_{2}\right) x_{2}+\frac{\left(1-r_{3}\right) \alpha_{2} \xi_{W} \psi}{\alpha_{1} \xi_{D}} x_{4}-\xi_{W} x_{3} \\
& \frac{d x_{4}}{d t}=f_{4}=\xi_{D}\left(1-r_{2}\right) x_{2}-\xi_{D} x_{4} \\
& \frac{d x_{5}}{d t}=f_{5}=\gamma x_{2}-\mu x_{5}
\end{aligned}
$$

The Jacobian of the system (3.4) at EE, $\varepsilon_{1}$, is given by 


$$
J\left(\varepsilon_{0}\right)=\left(\begin{array}{ccccc}
-\mu & \frac{-\beta_{I} \Lambda}{\mu} & \frac{-\left(1-r_{1}\right) \beta_{W} \Lambda}{\mu} & \frac{-\left(1-r_{3}\right) \beta_{D} \Lambda}{\mu} & 0 \\
0 & \frac{\beta_{I} \Lambda}{\mu}-\gamma-\mu & \frac{\left(1-r_{1}\right) \beta_{W} \Lambda}{\mu} & \frac{\left(1-r_{3}\right) \beta_{D} \Lambda}{\mu} & 0 \\
0 & \xi_{W}\left(1-r_{2}\right) & -\xi_{W} & \frac{\left(1-r_{3}\right) \alpha_{2} \xi_{W} \psi}{\alpha_{1} \xi_{D}} & 0 \\
0 & \xi_{D}\left(1-r_{2}\right) & 0 & -\xi_{D} & 0 \\
0 & \gamma & 0 & 0 & -\mu
\end{array}\right)
$$

Then, let

$$
J_{1}=\frac{\Lambda}{\mu}, \quad J_{2}=\frac{\left(1-r_{1}\right) \beta_{W} \Lambda}{\mu}, \quad J_{3}=\frac{\left(1-r_{3}\right) \beta_{D} \Lambda}{\mu}, \quad \text { and } \quad J_{4}=\frac{\left(1-r_{3}\right) \alpha_{2} \xi_{W} \psi}{\alpha_{1} \xi_{D}}
$$

I reduce $J\left(\varepsilon_{0}\right)$ to be

$$
J\left(\varepsilon_{0}\right)=\left(\begin{array}{ccccc}
-\mu & -J_{1} \beta_{I} & -J_{2} & -J_{3} & 0 \\
0 & J_{1} \beta_{I}-\gamma-\mu & J_{2} & J_{3} & 0 \\
0 & \xi_{W}\left(1-r_{2}\right) & -\xi_{W} & J_{4} & 0 \\
0 & \xi_{D}\left(1-r_{2}\right) & 0 & -\xi_{D} & 0 \\
0 & \gamma & 0 & 0 & -\mu
\end{array}\right)
$$

I rewrite $R_{0}$ as follow:

$$
\begin{aligned}
& R_{0}=\frac{\Lambda\left(\beta_{I} \alpha_{1} \xi_{D}+\beta_{W}\left(1-r_{1}\right)\left(1-r_{2}\right) \alpha_{1} \xi_{D}+\beta_{W}\left(1-r_{1}\right)\left(1-r_{2}\right)\left(1-r_{3}\right) \alpha_{2} \psi+\beta_{D}\left(1-r_{2}\right)\left(1-r_{3}\right) \alpha_{1} \xi_{D}\right)}{\mu(\gamma+\mu) \alpha_{1} \xi_{D}} \\
& \beta_{I}=R_{0} \mu(\gamma+\mu)-\Lambda \beta_{D}\left(1-r_{2}\right)\left(1-r_{3}\right)-\frac{\Lambda \beta_{W}\left(1-r_{1}\right)\left(1-r_{2}\right)\left(\alpha_{2} \psi+\alpha_{1} \xi_{D}-\alpha_{2} \psi r_{3}\right)}{\alpha_{1} \xi_{D}}
\end{aligned}
$$


Consider the case when $R_{0}=1$. Suppose, further that $\beta_{I}=\beta_{I}^{*}$ is chosen as a bifurcation parameter. Solving for $\beta_{I}$ from $R_{0}=1$ gives

$$
\begin{aligned}
& \beta_{I}^{*}=\mu(\gamma+\mu)-\Lambda \beta_{D}\left(1-r_{2}\right)\left(1-r_{3}\right)-\frac{\Lambda \beta_{W}\left(1-r_{1}\right)\left(1-r_{2}\right)\left(\alpha_{2} \psi+\alpha_{1} \xi_{D}-\alpha_{2} \psi r_{3}\right)}{\alpha_{1} \xi_{D}} \\
& \beta_{I}^{*}=D_{1}-D_{2}-D_{3}
\end{aligned}
$$

where

$$
\begin{aligned}
& D_{1}=\mu(\gamma+\mu) \\
& D_{2}=\Lambda \beta_{D}\left(1-r_{2}\right)\left(1-r_{3}\right) \\
& D_{3}=\frac{\Lambda \beta_{W}\left(1-r_{1}\right)\left(1-r_{2}\right)\left(\alpha_{2} \psi+\alpha_{1} \xi_{D}-\alpha_{2} \psi r_{3}\right)}{\alpha_{1} \xi_{D}}
\end{aligned}
$$

The right eigenvectors of $J\left(\beta_{I}^{*}\right)$ are given by $w=\left[w_{1}, w_{2}, w_{3}, w_{4}, w_{5}\right]^{T}$, where

$$
\left(\begin{array}{ccccc}
-\mu & -J_{1} \beta_{I} & -J_{2} & -J_{3} & 0 \\
0 & J_{1} \beta_{I}-\gamma-\mu & J_{2} & J_{3} & 0 \\
0 & \xi_{W}\left(1-r_{2}\right) & -\xi_{W} & J_{4} & 0 \\
0 & \xi_{D}\left(1-r_{2}\right) & 0 & -\xi_{D} & 0 \\
0 & \gamma & 0 & 0 & -\mu
\end{array}\right)\left(\begin{array}{c}
w_{1} \\
w_{2} \\
w_{3} \\
w_{4} \\
w_{5}
\end{array}\right)=\mathbf{0}
$$

I obtain

$$
w_{1}=\frac{-\left(J_{1} \beta_{I}^{*} w_{2}+J_{2} w_{3}+J_{3} w_{4}\right)}{\mu}<0,
$$




$$
\begin{aligned}
& w_{2}>0, \\
& w_{3}=\frac{\xi_{W}\left(1-r_{2}\right) w_{2}+J_{4} w_{4}}{\xi_{W}}>0, \\
& w_{4}=\left(1-r_{2}\right) w_{2}>0, \\
& w_{5}=\frac{\gamma w_{2}}{\mu}>0
\end{aligned}
$$

Further, $J\left(\beta_{I}^{*}\right)$ has a left eigenvector $v=\left[v_{1}, v_{2}, v_{3}, v_{4}, v_{5}\right]$, where

$$
\left(\begin{array}{lllll}
v_{1} & v_{2} & v_{3} & v_{4} & v_{5}
\end{array}\right)\left(\begin{array}{ccccc}
-\mu & -J_{1} \beta_{I} & -J_{2} & -J_{3} & 0 \\
0 & J_{1} \beta_{I}-\gamma-\mu & J_{2} & J_{3} & 0 \\
0 & \xi_{W}\left(1-r_{2}\right) & -\xi_{W} & J_{4} & 0 \\
0 & \xi_{D}\left(1-r_{2}\right) & 0 & -\xi_{D} & 0 \\
0 & \gamma & 0 & 0 & -\mu
\end{array}\right)=\mathbf{0}
$$

I obtain

$$
\begin{aligned}
& v_{1}=0, \\
& v_{2}>0, \\
& v_{3}>0,
\end{aligned}
$$




$$
\begin{aligned}
& v_{4}=\frac{J_{3} v_{2}+J_{4} v_{3}}{\xi_{D}} \\
& v_{5}=0
\end{aligned}
$$

The transformed system (4.18), with $\beta_{I}=\beta_{I}^{*}$, has at least one non-hyperbolic equilibrium point because the linearized system has at least one eigenvalue with zero real part. Thus, the center manifold theory can be applied to analyze the dynamics of (4.18) with $\beta_{I}=\beta_{I}^{*}$. The following theorem [14] will be applied to show locallyasymptotically stable (LAS) of the endemic equilibrium (EE) of the transformed system (4.18).

Theorem 4. (Castillo-Chavez and Song [14])

Consider the following general system of ordinary differential equations with parameter $\phi$ :

$$
\frac{d x}{d t}=f(x, \phi), \quad f: \mathbb{R}^{n} \times \mathbb{R} \rightarrow \mathbb{R}, \quad f \in \mathbb{C}^{2}\left(\mathbb{R}^{n} \times \mathbb{R}\right)
$$

where 0 is an equilibrium of the system, that is $f(0, \phi)=0$ for all $\phi$, and assume that

A1. $A=D_{x} f(0,0)=\left(\frac{\partial f_{i}}{\partial x_{j}}(0,0)\right)$ is linearization matrix of the system around the equilibrium 0 with $\phi$ evaluated at 0 . Zero is a simple eigenvalues of $A$ and other eigenvalues of $A$ have negative real parts;

A2. Matrix $A$ has a right eigenvectors $w$ and a left eigenvector $v$ corresponding to the zero eigenvalue. 
Let $f_{k}$ be the $k^{\text {th }}$ component of $f$ and

$$
\begin{aligned}
& a=\sum_{k, i, j=1}^{n} u_{k} v_{i} v_{j} \frac{\partial^{2} f_{k}}{\partial x_{i} \partial x_{j}}(0,0), \\
& b=\sum_{k, i=1}^{n} u_{k} v_{i} \frac{\partial^{2} f_{k}}{\partial x_{i} \partial \phi}(0,0) .
\end{aligned}
$$

The local dynamics of the system around 0 is totally governed by $a$ and $b$.

i. $a>0, b>0$. When $\phi<0$ with $|\phi| \ll 1,0$ is locally asymptotically stable and there exists a positive unstable equilibrium; when $0<\phi \ll 1$, 0 is locally asymptotically stable equilibrium;

ii. $a<0, b<0$. When $\phi<0$ with $|\phi| \ll 1$, 0 is unstable; when $0<\phi \ll 1$, 0 is locally asymptotically stable equilibrium, and there exists a positive unstable equilibrium;

iii. $a>0, b<0$. When $\phi<0$ with $|\phi| \ll 1,0$ is unstable, there exists a locally asymptotically stable negative equilibrium; when $0<\phi \ll 1$, 0 is unstable, and a positive unstable equilibrium appears;

iv. $a<0, b>0$. When $\phi$ changes from negative to positive, 0 changes its stability from stable to unstable. Correspondingly a negative unstable equilibrium becomes positive and locally asymptotically stable.

To apply the above theorem, the following computations of $a$ and $b$ are neccessary. For the system (4.18), the associated non-zero partial derivatives of the right hand 
side function $\left(f_{i}\right)$ are given by

$$
\begin{aligned}
& \frac{\partial^{2} f_{1}}{\partial x_{1} x_{3}}=-\left(1-r_{1}\right) \beta_{W} \\
& \frac{\partial^{2} f_{1}}{\partial x_{1} x_{4}}=\left(1-r_{3}\right) \beta_{D} \\
& \frac{\partial^{2} f_{1}}{\partial x_{1} x_{2}}=-\beta_{I} \\
& \frac{\partial^{2} f_{2}}{\partial x_{1} x_{3}}=\left(1-r_{1}\right) \beta_{W} \\
& \frac{\partial^{2} f_{2}}{\partial x_{1} x_{4}}=\left(1-r_{3}\right) \beta_{D} \\
& \frac{\partial^{2} f_{2}}{\partial x_{1} x_{2}}=\beta_{I} \\
& \frac{\partial^{2} f_{2}}{\partial x_{2} \phi}=x_{1}=\frac{\Lambda}{\mu} \\
& \frac{\partial^{2} f_{1}}{\partial x_{1} \phi}=-x_{1}=-\frac{\Lambda}{\mu} \\
& x_{1} \phi
\end{aligned}
$$

Hence, it follows from the above expressions that

$$
a=\sum_{k, i, j=1}^{5} u_{k} v_{i} v_{j} \frac{\partial^{2} f_{k}}{\partial x_{i} \partial x_{j}}(0,0)
$$




$$
\begin{aligned}
& =\sum_{k, i, j=1}^{5} u_{k} v_{i} v_{j} \frac{\partial^{2} f_{1}}{\partial x_{i} \partial x_{j}}(0,0)+\sum_{k, i, j=1}^{5} u_{k} v_{i} v_{j} \frac{\partial^{2} f_{2}}{\partial x_{i} \partial x_{j}}(0,0) \\
& =\sum_{k, i, j=1}^{5} u_{k} v_{i} v_{j} \frac{\partial^{2} f_{2}}{\partial x_{i} \partial x_{j}}(0,0) \\
& =v_{1} w_{1} w_{3}\left(1-r_{1}\right) \beta_{W}+v_{2} w_{1} w_{4}\left(1-r_{3}\right) \beta_{D}+v_{2} w_{1} w_{2} \beta_{I} \\
& =v_{2} w_{1}\left(w_{3}\left(1-r_{1}\right) \beta_{W}+w_{4}\left(1-r_{3}\right) \beta_{D}+w_{2} \beta_{I}\right) \\
& <0
\end{aligned}
$$

and

$$
\begin{aligned}
& b=\sum_{k, i=1}^{5} u_{k} v_{i} \frac{\partial^{2} f_{k}}{\partial x_{i} \partial \phi}(0,0) \\
& =\sum_{k, i=1}^{5} u_{k} v_{i} \frac{\partial^{2} f_{1}}{\partial x_{i} \partial \phi}(0,0)+\sum_{k, i=1}^{5} u_{k} v_{i} \frac{\partial^{2} f_{2}}{\partial x_{i} \partial \phi}(0,0) \\
& =v_{1} w_{2} \frac{\partial^{2} f_{1}}{\partial x_{2} \phi}+v_{2} w_{2} \frac{\partial^{2} f_{2}}{\partial x_{2} \phi} \\
& =v_{2} w_{2} \frac{\Lambda}{\mu} \\
& >0
\end{aligned}
$$

Thus, $a<0$ and $b>0$. Hence, by Theorem 4 above, the unique endemic equilibrium of the system (4.18), which exists whenever $R_{0}>1$, is LAS whenever $R_{0}>1$ and $\beta_{I}^{*}<\beta_{I}$ with $\beta_{I}$ close to $\beta_{I}^{*}$.

In the case of $R_{0}>1$, numerous simulations of the model (3.4) show that $\varepsilon_{1}$ is globally-asymptotically stable. Thus, I make conjecture as follows: 
Conjecture 1. The unique endemic equilibrium of the model (3.4) is globallyasymptotically stable in $\Omega$, whenever $R_{0}>1$.

\subsection{Summary of the fundamental properties of the SIWDR model}

This chapter provides a rigorous mathematical study of the dynamics of the water and sanitation (WatSan) deficit and its public health impact in a community. The theoretical analysis of the SIWDR model reveals that the disease-free equilibrium is globally-asymptotically stable whenever a certain threshold (known as the reproduction number $\left.\left(R_{0}\right)\right)$ is less than one. However, the disease-free equilibrium is unstable if the threshold is greater than one. This implies that the number of infected population will be effectively eliminated at the steady-state, if the threshold is made to be less than one. In addition, I investigate an endemic equilibrium, and I prove that the endemic equilibrium is locally-asymptotically stable whenever the threshold is more than one. 


\section{Chapter 5}

\section{Optimal Control for SIWDR Model}

This chapter explains how improving WatSan services (drinking water supply-DWS, wastewater and sewage treatment-WST, and municipal solid waste managementMSW) can serve as a long-term control mechanism of enteric diarrheal disease (EDD). Increasing DWS, WST, and MSW services helps to reduce the cases of the EDD in the long run. Optimal control theory is applied to the SIWDR (Susceptible-InfectedWater-Dumpsite-Recovered) model. Then, the optimal control is determined by the Pontryagin's Maximum Principle, the optimal solution is assessed, and cost index is defined. The effects of 8 different control strategies (1. No control 2. DWS 3. WST 4. MSW 5. DWS and WST 6. DWS and MSW 7. WST and MSW 8. DWS, WST, and MSW) are evaluated in 2 cases $\left(1 . R_{0}>12 . R_{0}<1\right)$ by numerical simulations. Lastly, I compare the optimal solution obtained from numerical simulations with other allocation strategies, such as the strategy that allocates budget equally among WatSan services. However, the result shows that the optimal solution reduces the number of infected people more than any other strategies. 


\section{$5.1 \quad$ Introduction}

Even though clean water and basic sanitation are necessary for health of all people [58] [24], there are quite a number of people who do not have adequate water and sanitation services, especially those in lower income countries (LICs). Approximately 1.6 million people die every year from diarrheal disease. Ninety percent of them are children under 5 years of age [85]. Examples of diseases caused by contaminated water and lack of access to Watsan services include acute respiratory infections, and schistosomiasis [47] [26]. These health problems are urgent because they result in high loss of patients' opportunities and income obtained for work.

Poor WatSan services strengthen the many pathways to enteric pathogens transmission. The pathogens can be passed from fecal matter of infected people to the environment, which can finally get into healthy individuals through drinking unclean water and food. This fecal-oral disease transmission (fecal-oral routes), however, can be prevented in the long run by improving WatSan services, i.e., adding DWS, and WST services [38]. MSW services are added in this case also due to its limited formal management in many LICs. That is to say, dumpsites are one of the most important and largest reservoirs of pathogens in these LICs. Pathogens would travel from dumpsites through leachate to unprotected groundwater sources or surface water as runoff [40] [28]. Individuals, such as waste pickers, can become infected when they contact the infected water or waste directly [42] [1] [62] [29].

Mathematical models are important analysis tools in the control of infectious disease. The SIR (Susceptible-Infected-Recovered) model, the ordinary differential equations (ODE) model, invented by Kermack and Mckendrick (1927) [37] is the basic modelling techniques used in the control of infectious disease. The SIR model is used to analyze many infectious disease such as influenza [20] [53] and cholera [48]. The populations in the SIR model are divided into the three distinct groups. Susceptible $(S)$ represents individuals who may become infected if they have contact with an 


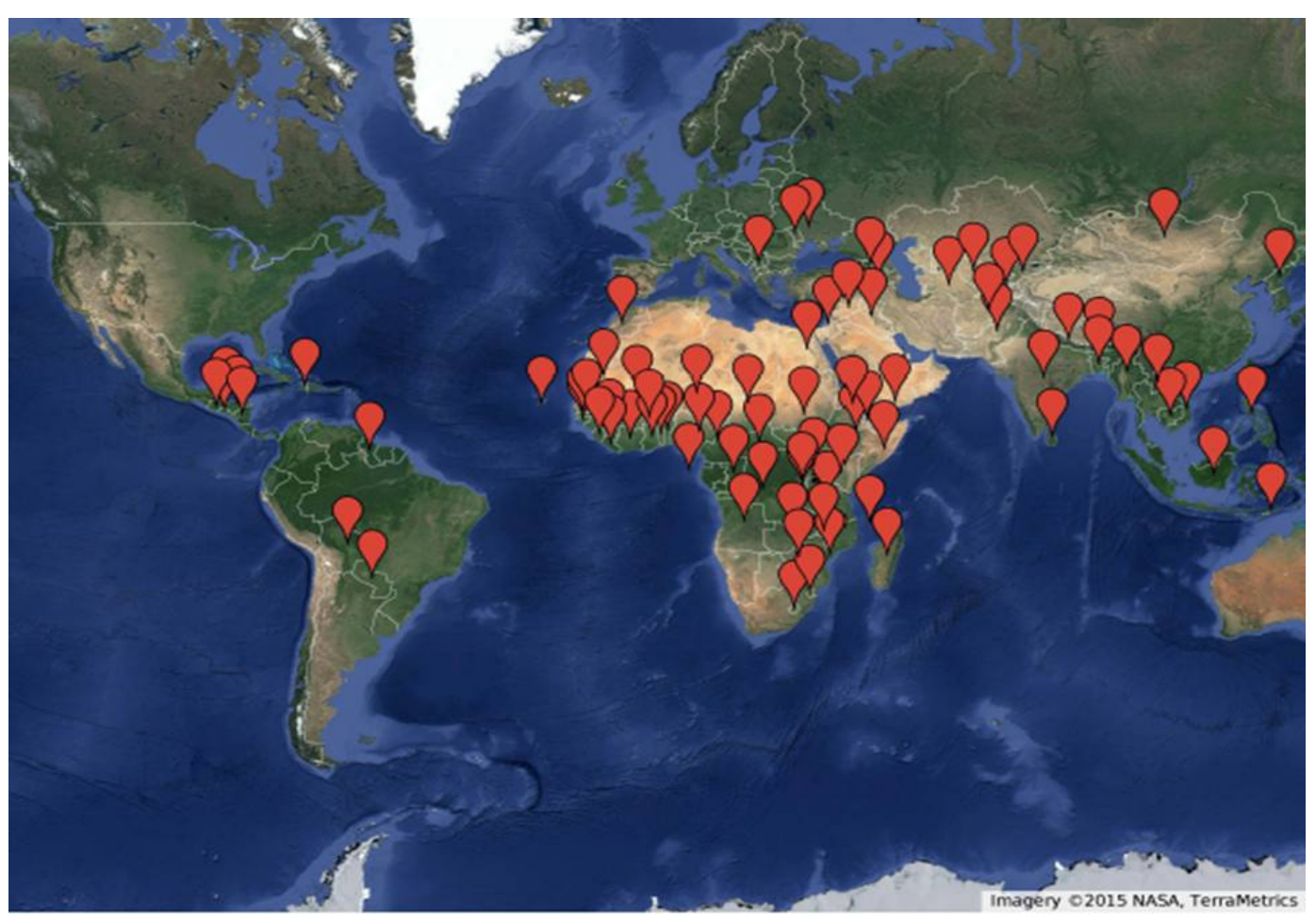

Figure 5.1: Lower income countries map.

infected individual. Infected $(I)$ represents individuals who are currently infected and can spread the disease to $S$ whom they contact. Recovered $(R)$ represents individuals who are previously infected, but are currently recovered.

The use of mathematical model allows us to evaluate different control strategies, including the ones which are difficult to test in human population. For example, how much oral cholera vaccines (OCVs) coverage would be necessary for cholera control?

In order to obtain the best control strategy, optimal control theory is used. The general description of optimal control problems, analytical results, examples are described by Lenhart and Workman (2007) [41]. More specific control strategies, such as vaccines [4] [5] [77] [36], health-promotion campaigns [5] [12], isolation (health care) [19], and quarantine and screening [5] [36] were fully described in the literature. These control strategies give me an idea to use WatSan services as controls to reduce EDDs transmission. 


\subsection{Systems modeling}

In this chapter the SIWDR (Susceptible-Infected-Water-Dumpsite-Recovered) model is considered aiming at the control of EDDs spreading. The optimal control theory is used to best allocate the budget under constraints.

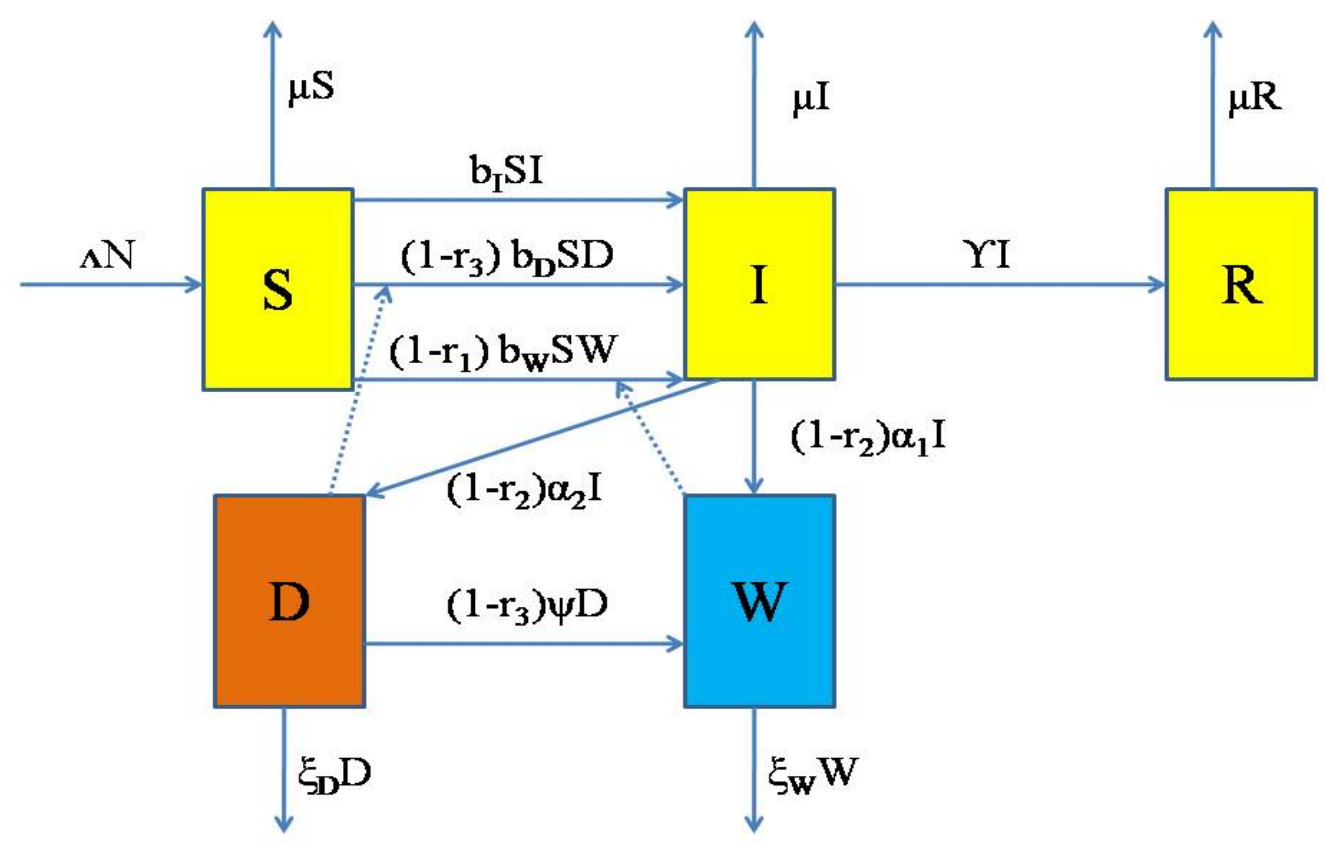

Figure 5.2: Flow diagram for the SIWDR model

The system of nonlinear differential equations is

$$
\begin{aligned}
\dot{S} & =\Lambda N-\left(1-r_{1}\right) b_{W} S(t) W(t)-\left(1-r_{3}\right) b_{D} S(t) D(t)-b_{I} S(t) I(t)-\mu S \\
\dot{I} & =\left(1-r_{1}\right) b_{W} S(t) W(t)+\left(1-r_{3}\right) b_{D} S(t) D(t)+b_{I} S(t) I(t)-\gamma I(t)-\mu I(t) \\
\dot{W} & =\left(1-r_{2}\right) \alpha_{1} I(t)+\left(1-r_{3}\right) \psi D(t)-\xi_{W} W(t) \\
\dot{D} & =\left(1-r_{2}\right) \alpha_{2} I(t)-\xi_{D} D(t) \\
\dot{R} & =\gamma I(t)-\mu R(t)
\end{aligned}
$$


For ease of analysis, the system model (5.1) is scaled to be dimensionless variables. The scaled version of the system model (5.1) is

$$
\begin{aligned}
& \dot{s}=\Lambda-\left(1-r_{1}\right) \beta_{W} s(t) w(t)-\left(1-r_{3}\right) \beta_{D} s(t) d(t)-\beta_{I} s(t) i(t)-\mu s(t) \\
& \dot{i}=\left(1-r_{1}\right) \beta_{W} s(t) w(t)+\left(1-r_{3}\right) \beta_{D} s(t) d(t)+\beta_{I} s(t) i(t)-\gamma i(t)-\mu i(t) \\
& \dot{w}=\xi_{W}\left(1-r_{2}\right) i(t)+\frac{\left(1-r_{3}\right) \alpha_{2} \xi_{W} \psi}{\alpha_{1} \xi_{D}} d(t)-\xi_{W} w(t) \\
& \dot{d}=\xi_{D}\left(1-r_{2}\right) i(t)-\xi_{D} d(t) \\
& \dot{r}=\gamma i(t)-\mu r(t)
\end{aligned}
$$

I consider the system model (5.2) with initial conditions:

$$
s(0)=s_{0}, \quad i(0)=i_{0}, \quad w(0)=w_{0}, \quad d(0)=d_{0}, \quad r(0)=r_{0}
$$

This study controls WatSan services and observes what happens to the number of cholera infected population. $u_{1}(t), u_{2}(t)$, and $u_{3}(t)$ are used to represent DWS, WST, and MSW services, in the systems model (equation (5.2)), respectively.

$$
\begin{aligned}
& \dot{s}=\Lambda-\left(1-r_{1}\right) \beta_{W} s(t) w(t)-\left(1-r_{3}\right) \beta_{D} s(t) d(t)-\beta_{I} s(t) i(t)-\mu s(t) \\
& \dot{i}=\left(1-r_{1}-u_{1}(t)\right) \beta_{W} s(t) w(t)+\left(1-r_{3}-u_{3}(t)\right) \beta_{D} s(t) d(t)+\beta_{I} s(t) i(t)-\gamma i(t)-\mu i(t) \\
& \dot{w}=\xi_{W}\left(1-r_{2}-u_{2}(t)\right) i(t)+\frac{\left(1-r_{3}-u_{3}(t)\right) \alpha_{2} \xi_{W} \psi}{\alpha_{1} \xi_{D}} d(t)-\xi_{W} w(t) \\
& \dot{d}=\xi_{D}\left(1-r_{2}-u_{2}(t)\right) i(t)-\xi_{D} d(t) \\
& \left.\dot{r}=\gamma i(t)-\mu r(t)+u_{1}(t) \beta_{W} s(t) w(t)+u_{3}(t)\right) \beta_{D} s(t) d(t)
\end{aligned}
$$

with initial conditions equal to the initial conditions above and with the bounds:

$$
u_{1 \min } \leq u_{1}(t) \leq u_{1 \max }, \quad u_{2 \min } \leq u_{2}(t) \leq u_{2 \max }, \quad u_{3 \min } \leq u_{3}(t) \leq u_{3 \max }
$$




\subsection{Existence of solution of the control system}

Before I discuss about the optimal control in the next section, I need to show the existence of the solution of the control system first. I rewrite (5.3) in the following form:

$$
\dot{x}=A x+F(x)
$$

where a dot $(\cdot)$ represents differentiation with respect to time $(t)$.

where

$$
\begin{aligned}
& x=(s(t) \quad i(t) \quad w(t) \quad d(t) \quad r(r))^{T}, \\
& A=\left[\begin{array}{ccccc}
-\mu & 0 & 0 & 0 & 0 \\
0 & -\gamma-\mu & 0 & 0 & 0 \\
0 & \xi_{W}\left(1-r_{2}-u_{2}(t)\right) & -\xi_{W} & \frac{\left(1-r_{3}-u_{3}(t)\right) \alpha_{2} \xi_{W} \psi}{\alpha_{1} \xi_{D}} & 0 \\
0 & \xi_{D}\left(1-r_{2}-u_{2}(t)\right) & 0 & -\xi_{D} & 0 \\
0 & \gamma & 0 & 0 & -\mu
\end{array}\right]
\end{aligned}
$$

$$
F(x)=\left[\begin{array}{c}
\Lambda-\left(1-r_{1}\right) \beta_{W} s(t) w(t)-\left(1-r_{3}\right) \beta_{D} s(t) d(t)-\beta_{I} s(t) i(t) \\
\left(1-r_{1}-u_{1}(t)\right) \beta_{W} s(t) w(t)+\left(1-r_{3}-u_{3}(t)\right) \beta_{D} s(t) d(t)+\beta_{I} s(t) i(t) \\
0 \\
0 \\
u_{1}(t) \beta_{W} s(t) w(t)+u_{3}(t) \beta_{D} s(t) d(t)
\end{array}\right]
$$


I set

$$
G(x)=A x+F(x)
$$

The second term on the right hand side of (5.5) satisfies

$$
F\left(x_{1}\right)-F\left(x_{2}\right)=
$$

$$
\left[\begin{array}{c}
\Lambda-\left(1-r_{1}\right) \beta_{W} s_{1}(t) w_{1}(t)-\left(1-r_{3}\right) \beta_{D} s_{1}(t) d_{1}(t)-\beta_{I} s_{1}(t) i_{1}(t) \\
\left(1-r_{1}-u_{1}(t)\right) \beta_{W} s_{1}(t) w_{1}(t)+\left(1-r_{3}-u_{3}(t)\right) \beta_{D} s_{1}(t) d_{1}(t)+\beta_{I} s_{1}(t) i_{1}(t) \\
0 \\
0 \\
u_{1}(t) \beta_{W} s_{1}(t) w_{1}(t)+u_{3}(t) \beta_{D} s_{1}(t) d_{1}(t)
\end{array}\right]
$$$$
-\left[\begin{array}{c}
\Lambda-\left(1-r_{1}\right) \beta_{W} s_{2}(t) w_{2}(t)-\left(1-r_{3}\right) \beta_{D} s_{2}(t) d_{2}(t)-\beta_{I} s_{2}(t) i_{2}(t) \\
\left(1-r_{1}-u_{1}(t)\right) \beta_{W} s_{2}(t) w_{2}(t)+\left(1-r_{3}-u_{3}(t)\right) \beta_{D} s_{2}(t) d_{2}(t)+\beta_{I} s_{2}(t) i_{2}(t) \\
0 \\
0 \\
u_{1}(t) \beta_{W} s_{2}(t) w_{2}(t)+u_{3}(t) \beta_{D} s_{2}(t) d_{2}(t)
\end{array}\right]
$$

$F\left(x_{1}\right)-F\left(x_{2}\right) \leq \max \left\{s_{1}, s_{2}\right\} \times$ 


$$
\left[\begin{array}{c}
-\left(1-r_{1}\right) \beta_{W}\left|w_{1}(t)-w_{2}(t)\right|-\left(1-r_{3}\right) \beta_{D}\left|d_{1}(t)-d_{2}(t)\right|-\beta_{I}\left|i_{1}(t)-i_{2}(t)\right| \\
\left(1-r_{1}-u_{1}(t)\right) \beta_{W}\left|w_{1}(t)-w_{2}(t)\right|+\left(1-r_{3}-u_{3}(t)\right) \beta_{D}\left|d_{1}(t)-d_{2}(t)\right|+\beta_{I}\left|i_{1}(t)-i_{2}(t)\right| \\
0 \\
0 \\
u_{1}(t) \beta_{W}\left|w_{1}(t)-w_{2}(t)\right|+u_{3}(t) \beta_{D}\left|d_{1}(t)-d_{2}(t)\right|
\end{array}\right]
$$

$$
\begin{aligned}
\mid F( & \left.x_{1}\right)-F\left(x_{2}\right) \mid \leq \max \left\{s_{1}, s_{2}\right\} \times \\
& \left\{\left(1-r_{1}\right)^{2} \beta_{W}^{2}\left|w_{1}(t)-w_{2}(t)\right|^{2}+\left(1-r_{3}\right)^{2} \beta_{D}^{2}\left|d_{1}(t)-d_{2}(t)\right|^{2}+\beta_{I}^{2}\left|i_{1}(t)-i_{2}(t)\right|^{2}+\right. \\
& 2\left(1-r_{1}\right) \beta_{W}\left|w_{1}(t)-w_{2}(t)\right|\left(1-r_{3}\right) \beta_{D}\left|d_{1}(t)-d_{2}(t)\right|+ \\
& 2\left(1-r_{1}\right) \beta_{W}\left|w_{1}(t)-w_{2}(t)\right| \beta_{I}\left|i_{1}(t)-i_{2}(t)\right|+ \\
& 2\left(1-r_{3}\right) \beta_{D}\left|d_{1}(t)-d_{2}(t)\right| \beta_{I}\left|i_{1}(t)-i_{2}(t)\right|+ \\
& \left(1-r_{1}-u_{1}(t)\right)^{2} \beta_{W}^{2}\left|w_{1}(t)-w_{2}(t)\right|^{2}+\left(1-r_{3}-u_{3}(t)\right)^{2} \beta_{D}^{2}\left|d_{1}(t)-d_{2}(t)\right|^{2}+\beta_{I}^{2} \mid i_{1}(t)- \\
\left.i_{2}(t)\right|^{2}+ & 2\left(1-r_{1}-u_{1}(t)\right) \beta_{W}\left|w_{1}(t)-w_{2}(t)\right|\left(1-r_{3}-u_{3}(t)\right) \beta_{D}\left|d_{1}(t)-d_{2}(t)\right|+ \\
& 2\left(1-r_{1}-u_{1}(t)\right) \beta_{W}\left|w_{1}(t)-w_{2}(t)\right| \beta_{I}\left|i_{1}(t)-i_{2}(t)\right|+ \\
& 2\left(1-r_{3}-u_{3}(t)\right) \beta_{D}\left|d_{1}(t)-d_{2}(t)\right| \beta_{I}\left|i_{1}(t)-i_{2}(t)\right|+ \\
& u_{1}(t)^{2} \beta_{W}^{2}\left|w_{1}(t)-w_{2}(t)\right|^{2}+u_{3}(t)^{2} \beta_{D}^{2}\left|d_{1}(t)-d_{2}(t)\right|^{2} \\
& \left.2 u_{1}(t) \beta_{W}\left|w_{1}(t)-w_{2}(t)\right| u_{3}(t) \beta_{D}\left|d_{1}(t)-d_{2}(t)\right|\right\}^{\frac{1}{2}}
\end{aligned}
$$

$$
\left|F\left(x_{1}\right)-F\left(x_{2}\right)\right| \leq \max \left\{s_{1}, s_{2}\right\} \times
$$$$
\left\{M_{1}\left|w_{1}(t)-w_{2}(t)\right|^{2}+M_{2}\left|d_{1}(t)-d_{2}(t)\right|^{2}+M_{3}\left|i_{1}(t)-i_{2}(t)\right|^{2}+\right.
$$$$
M_{4} \times \max \left\{\left|w_{1}(t)-w_{2}(t)\right|,\left|d_{1}(t)-d_{2}(t)\right|\right\}^{2}+
$$$$
M_{5} \times \max \left\{\left|w_{1}(t)-w_{2}(t)\right|,\left|i_{1}(t)-i_{2}(t)\right|\right\}^{2}+
$$$$
\left.M_{6} \times \max \left\{\left|d_{1}(t)-d_{2}(t)\right|,\left|i_{1}(t)-i_{2}(t)\right|\right\}^{2}\right\}^{\frac{1}{2}}
$$ 
where,

$$
\begin{aligned}
& M_{1}=\left(1-r_{1}\right)^{2} \beta_{W}^{2}+\left(1-r_{1}-u_{1}(t)\right)^{2} \beta_{W}^{2}+u_{1}(t)^{2} \beta_{W}^{2} \\
& M_{2}=\left(1-r_{3}\right)^{2} \beta_{D}^{2}+\left(1-r_{3}-u_{3}(t)\right)^{2} \beta_{D}^{2}+u_{3}(t)^{2} \beta_{D}^{2} \\
& M_{3}=2 \beta_{I}^{2} \\
& M_{4}=2\left(1-r_{1}\right) \beta_{W}\left(1-r_{3}\right) \beta_{D}+2\left(1-r_{1}-u_{1}(t)\right) \beta_{W}\left(1-r_{3}-u_{3}(t)\right) \beta_{D}+2 u_{1}(t) \beta_{W} u_{3}(t) \beta_{D} \\
& M_{5}=2\left(1-r_{1}\right) \beta_{W} \beta_{I}+2\left(1-r_{1}-u_{1}(t)\right) \beta_{W} \beta_{I} \\
& M_{6}=2\left(1-r_{3}\right) \beta_{D} \beta_{I}+2\left(1-r_{3}-u_{3}(t)\right) \beta_{D} \beta_{I}
\end{aligned}
$$

Then, I can combine the last three terms with the first three terms, and I can write the inequality in term of $\left|w_{1}(t)-w_{2}(t)\right|,\left|d_{1}(t)-d_{2}(t)\right|,\left|i_{1}(t)-i_{2}(t)\right|$. Thus, I obtain

$$
\left|F\left(x_{1}\right)-F\left(x_{2}\right)\right| \leq \hat{M}\left(\left|s_{1}(t)-s_{2}(t)\right|+\left|i_{1}(t)-i_{2}(t)\right|+\left|w_{1}(t)-w_{2}(t)\right|+\left|d_{1}(t)-d_{2}(t)\right|\right)
$$

where the positive constant $\hat{M}$ is independent of state variable $s(t), i(t), w(t)$, and $d(t)$. Also, I obtain

$$
\left|G\left(x_{1}\right)-G\left(x_{2}\right)\right| \leq M^{*}\left|x_{1}-x_{2}\right|
$$

where $M^{*}=\max \{\hat{M},\|A\|\}<\infty$. Hence, the function $G$ is uniformly Lipschitz continuous. From the property of function $G$, non-negativity of state variables, and the definition of control $u_{1}(t), u_{2}(t)$, and $u_{3}(t)$, I conclude that the solution of the system (5.3) exists.

\subsection{Optimal control strategy}

The definition of cost index is as follow: 


$$
\begin{aligned}
J\left(t, x(t), u_{1}(t), u_{2}(t), u_{3}(t)\right) & =\int_{t_{i}}^{t_{f}} L\left(t, x(t), u_{1}(t), u_{2}(t), u_{3}(t)\right) d t \\
& =\int_{t_{i}}^{t_{f}}\left[A i(t)+\frac{1}{2} \tau_{1} u_{1}(t)^{2}+\frac{1}{2} \tau_{2} u_{2}(t)^{2}+\frac{1}{2} \tau_{3} u_{3}(t)^{2}\right] d t
\end{aligned}
$$

with $A, \tau_{1}, \tau_{1}, \tau_{1}>0$ represnting the weights in the cost index. $t_{i} \geq 0$ and $t_{f}>0$ represent the fixed initial time and fixed final time of the control interval. The goal is to minimize the infected individuals using minimal control efforts.

Therefore, I consider the following problem.

Problem: statement of the problem is as follow:

$$
\begin{aligned}
& \min _{u_{1}, u_{2}, u_{3}} J\left(t, x(t), u_{1}(t), u_{2}(t), u_{3}(t)\right)=\min _{u_{1}, u_{2}, u_{3}} \int_{t_{i}}^{t_{f}} L\left(t, x(t), u_{1}(t), u_{2}(t), u_{3}(t)\right) d t \\
& \text { subject to } \quad \dot{x}=f\left(t, x(t), u_{1}(t), u_{2}(t), u_{3}(t)\right), \\
& x\left(t_{0}\right)=x_{0} .
\end{aligned}
$$

For our purposes, $f$ and $L$ are always differentiable in all arguments.

Let's define the Hamitonian function:

$$
H(\cdot)=L\left(t, x(t), u_{1}(t), u_{2}(t), u_{3}(t)\right)+\lambda(t)^{T} f\left(t, x(t), u_{1}(t), u_{2}(t), u_{3}(t)\right)
$$




$$
\begin{aligned}
& =\left(A i(t)+\frac{1}{2} \tau_{1} u_{1}(t)^{2}+\frac{1}{2} \tau_{2} u_{2}(t)^{2}+\frac{1}{2} \tau_{3} u_{3}(t)^{2}\right)+ \\
& \lambda_{1}(t)\left\{\Lambda-\left(1-r_{1}\right) \beta_{W} s(t) w(t)-\left(1-r_{3}\right) \beta_{D} s(t) d(t)-\beta_{I} s(t) i(t)-\mu s(t)\right\}+ \\
& \lambda_{2}(t)\left\{\left(1-r_{1}-u_{1}(t)\right) \beta_{W} s(t) w(t)+\left(1-r_{3}-u_{3}(t)\right) \beta_{D} s(t) d(t)+\beta_{I} s(t) i(t)-\right. \\
& \gamma i(t)-\mu i(t)\}+ \\
& \lambda_{3}(t)\left\{\xi_{W}\left(1-r_{2}-u_{2}(t)\right) i(t)+\frac{\left(1-r_{3}-u_{3}(t) \alpha_{2} \xi_{W} \psi\right.}{\alpha_{1} \xi_{D}} d(t)-\xi_{W} w(t)\right\}+ \\
& \lambda_{4}(t)\left\{\xi_{D}\left(1-r_{2}-u_{2}(t)\right) i(t)-\xi_{D} d(t)\right\}+ \\
& \lambda_{5}(t)\left\{\gamma i(t)-\mu r(t)+u_{1}(t) \beta_{W} s(t) w(t)+u_{3}(t) \beta_{D} s(t) d(t)\right\}
\end{aligned}
$$

where $\lambda$ is the Lagrange multiplier (adjoint variable).

Let $x^{*}$ be the optimal state trajectory.

Let $u_{1}^{*}, u_{2}^{*}, u_{3}^{*}$ be optimal controls.

Let $\lambda^{*}$ be corresponding Lagrange multiplier.

From the Pontryagin's maximum principle [57], the following result holds.

(1) All admissible controls $u_{1}^{*}(t), u_{2}^{*}(t), u_{3}^{*}(t)$

$H\left(x^{*}(t), u_{1}^{*}(t), u_{2}^{*}(t), u_{3}^{*}(t), \lambda^{*}(t)\right) \leq H\left(x^{*}(t), \overline{u_{1}}(t), \overline{u_{2}}(t), \overline{u_{3}}(t), \lambda^{*}(t)\right)$

(2) Costate equations

$$
\dot{\lambda}_{1}^{*}(t)=-\left.\frac{\partial H\left(x(t), u_{1}(t), u_{2}(t), u_{3}(t), \lambda(t)\right)}{\partial s(t)}\right|^{*}
$$




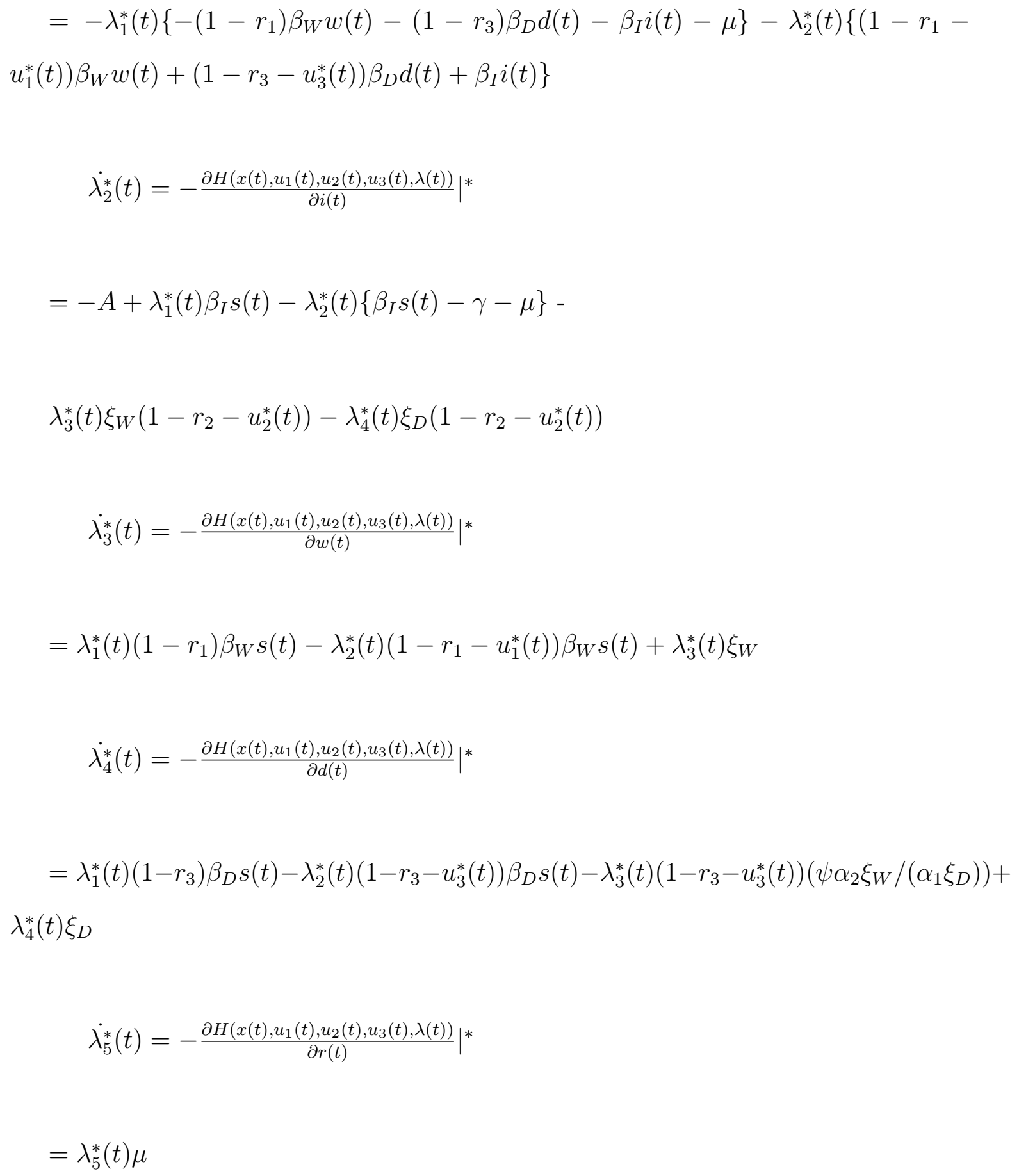

(3) Transversility condition

$$
\lambda_{1}\left(t_{f}\right)=\lambda_{2}\left(t_{f}\right)=\lambda_{3}\left(t_{f}\right)=\lambda_{4}\left(t_{f}\right)=\lambda_{5}\left(t_{f}\right)=0
$$


I consider the optimality condition as follow:

$$
\begin{aligned}
& \left.\frac{\partial H(\cdot)}{\partial u_{1}(t)}\right|^{*}=0 \Rightarrow u_{1}^{*}(t)=\frac{\lambda_{2}^{*}(t) \beta_{W} s(t) w(t)}{\tau_{1}} \\
& \left.\frac{\partial H(\cdot)}{\partial u_{2}(t)}\right|^{*}=0 \Rightarrow u_{2}^{*}(t)=\frac{\left(\lambda_{3}^{*}(t) \xi_{W}+\lambda_{4}^{*}(t) \xi_{D}\right) i(t)}{\tau_{2}} \\
& \left.\frac{\partial H(\cdot)}{\partial u_{2}(t)}\right|^{*}=0 \Rightarrow u_{3}^{*}(t)=\frac{\lambda_{2}^{*}(t) \beta_{D} d(t) s(t)+\left(\lambda_{3}^{*}(t) \alpha_{2} \psi \xi_{W} d(t)\right) /\left(\alpha_{1} \xi_{D}\right)}{\tau_{3}}
\end{aligned}
$$

Since I consider bounds on the controls, the optimal controls $u_{1}^{*}(t), u_{2}^{*}(t), u_{3}^{*}(t)$ must satisfy the following optimality condition:

$$
\begin{gathered}
u_{1}^{*}(t)= \begin{cases}u_{1 \min } & \text { if } \frac{\partial H(\cdot)}{\partial u_{1}(t)}>0 \\
\frac{\lambda_{2}^{*}(t) \beta_{W} s(t) w(t)}{\tau_{1}} & \text { if } \frac{\partial H(\cdot)}{\partial u_{1}(t)}=0 \\
u_{1 \max } & \text { if } \frac{\partial H(\cdot)}{\partial u_{1}(t)}<0\end{cases} \\
u_{2}^{*}(t)= \begin{cases}u_{2 \min } & \text { if } \frac{\partial H(\cdot)}{\partial u_{2}(t)}>0 \\
\frac{\left(\lambda_{3}^{*}(t) \xi_{W}+\lambda_{4}^{*}(t) \xi_{D}\right) i(t)}{\tau_{2}} & \text { if } \frac{\partial H(\cdot)}{\partial u_{2}(t)}=0 \\
u_{2 \max } & \text { if } \frac{\partial H(\cdot)}{\partial u_{2}(t)}<0\end{cases}
\end{gathered}
$$




$$
u_{3}^{*}(t)= \begin{cases}u_{3 \min } & \text { if } \frac{\partial H(\cdot)}{\partial u_{3}(t)}>0 \\ \frac{\lambda_{2}^{*}(t) \beta_{D} d(t) s(t)+\left(\lambda_{3}^{*}(t) \alpha_{2} \psi \xi_{W} d(t)\right) /\left(\alpha_{1} \xi_{D}\right)}{\tau_{3}} & \text { if } \frac{\partial H(\cdot)}{\partial u_{3}(t)}=0 \\ u_{3 \max } & \text { if } \frac{\partial H(\cdot)}{\partial u_{3}(t)}<0\end{cases}
$$

The numerical solution of the optimal control problem is discussed in the next section.

\subsection{Results and discussion}

Because solutions to the equation (5.3) cannot be obtained directly, I use a numerical method to find the optimal controls for $u_{1}, u_{2}$, and $u_{3}$. This is to best reduce the number of cholera infected population (referred in the equation (5.3)). Using the Pontryagin's maximum principle, I discover the necessary conditions used in the numerical method, Forward-Backward Sweep Method by Lenhart (2007). These conditions are needed to find the optimal controls for $u_{1}, u_{2}$, and $u_{3}$. In this numerical method, Matlab code is written by adapting from the code in Lenhart (2007)'s book, Optimal Control Applied to Biological Model [41]. Within the code, I apply the Runge-Kutta method to forwardly approximate solutions of the ordinary differential equation (equation (5.3)); while the value of $t$ starts from 0 to 60 . Then, I apply the Runge-Kutta method once again to backwardly approximate solutions of the costate equations; while the value of $t$ starts from 60 to 0 . Finally, the numerical solutions of the equation (5.3) are obtained. See details of the Matlab code in the Appendix C.

I use the values of parameters shown in the table 3.3 to observe what happens to the number of cholera infected population when putting WatSan-service control strategies (equation (5.3)). The values and plausible range explored are taken directly from relevant literature. Minimum and maximum values of each parameter are presented. 
These values will be used to represent the 2010-2015 cholera outbreak in Haiti.

The cost index were chosen in order to minimize the effect over the infected population with respect to the control $u_{1}, u_{2}$, and $u_{3}$.

$$
A=3, \quad \tau_{1}=1, \quad \tau_{2}=1, \quad \tau_{3}=1
$$

Also, a limitation on each control is as follow:

$$
0.001 \leq u_{1} \leq 0.365, \quad 0.001 \leq u_{2} \leq 0.775, \quad 0.001 \leq u_{3} \leq 0.900
$$

The lower bound for each control parameter is set to $0.1 \%$. This is because WatSan services are set to always be increased or stay the same. Upper bounds vary for each control parameter depending on WatSan coverage. However, for each control parameter, the upper bound cannot go above $1-r_{i}$, for $i=1,2$, and 3 . If the upper bound goes above this value, the value of $1-r_{i}-u_{i}$ will fall below 0 , and this violates the bound set for each parameter mentioned in the chapter 4 .

The initial conditions ${ }^{1}$ are:

$$
s_{0}=1-\frac{1}{10320000}, \quad i_{0}=\frac{1}{10320000}, \quad w_{0}=0, \quad d_{0}=0, \quad r_{0}=0
$$

Below, the reference situation is when there is no control. I want to see what happens to the number of infected population when no control exists. This way, various control strategies can be explored. The following percentage is evaluated as follows:

\footnotetext{
${ }^{1}$ The total population of Haiti is $10,320,000$.
} 


$$
k_{I}=\frac{\int_{t_{i}}^{t_{f}} I(t)-\int_{t_{i}}^{t_{f}} \hat{I}(t)}{\int_{t_{i}}^{t_{f}} \hat{I}(t)} \times 100
$$

where

$t_{i}$ is the fixed initial time $\left(t_{i}=0\right)$,

$t_{f}$ is the fixed final time $\left(t_{f}=60\right)$,

$I(t)$ is the infected population at time $t$ in one of the considered control situations,

$\hat{I}(t)$ is the infected population at time $t$ in the reference situation with no control.

Two cases are studied:

1. Case 1 is when $R_{0}>1$; endemic equilibrium.

2. Case 2 is when $R_{0}<1$; disease-free equilibrium.

In the case 1, I use the same values for each parameter used in the 2010-2015 cholera outbreak in Haiti (See the table 3.3). In the case 2, the values for each parameter are the same as in the case 1 , except the value of $\gamma$. I change the $\gamma$ value from 1.5 to 3.22 , so that $R_{0}$ falls below $1(0.99)$. This is to imitate what happens in reality during the cholera outbreak. During the outbreak, there will be an increase in vaccines that helps develop immunity in the exposed, but uninfected population. The vaccines lengthen the time it takes for the uninfected population to be infected, decreasing the $\frac{1}{\gamma}$ value (infectious period), which in turn, causing $R_{0}$ value to fall below 1 .

I test 8 different situations within each case: situations with 1) no control, 2) $u_{1}$, 3) $\left.\left.u_{2}, 4\right) u_{3}, 5\right) u_{1}$ and $\left.u_{2}, 6\right) u_{1}$ and $\left.u_{3}, 7\right) u_{2}$ and $u_{3}$, and 8) $u_{1}, u_{2}$, and $u_{3}$. See the table 5.1 below for details. $u_{1}, u_{2}$, and $u_{3}$ represent controls in DWS, WST, and MSW services, respectively. 
For case 1 ( $R_{0}>1$; endemic equilibrium), the total number of infected population is summarized in the table 5.1:

Table 5.1: Case 1: Comparison of number of infected population in the situation with no controls, and that with controls.

\begin{tabular}{|l|c|c|}
\hline Situation & Total number of infected population & $k_{I}$ \\
\hline 1. no controls & 0.3270 & 0.00 \\
\hline 2. $u_{1}$ & 0.1762 & -46.12 \\
\hline 3. $u_{2}$ & 0.3267 & -0.09 \\
\hline 4. $u_{3}$ & -2.66 \\
\hline 5. $u_{1}, u_{2}$ & 0.3183 & -46.21 \\
\hline 6. $u_{1}, u_{3}$ & 0.1759 & -46.18 \\
\hline 7. $u_{2}, u_{3}$ & 0.1760 & -2.75 \\
\hline 8. $u_{1}, u_{2}, u_{3}$ & 0.3180 & -46.25 \\
\hline
\end{tabular}

From the table 5.1, the last case, with controls in DWS, WST, and MSW services, results in the least number of infected population (-46.25\%). Also, $u_{1}$ or controls in DWS services is the most influential factor in reducing the number of infected population. This is seen in the situation $2,5,6$, and 8 where $u_{1}$ exists, and the total number of infected population is reduced in approximately the same amount. $u_{2}$ is the least influential factor $(-0.09 \%)$, and $u_{3}$ has small influence on the number of infected population $(-2.66 \%)$.

The figure 5.3 below shows the dynamic of all variables, $u_{1}, u_{2}$, and $u_{3}$ in the SIWDR model, in the situation 8.

The graphs of the Susceptible $(S)$, Infected $(I)$, and Recovered $(R)$ variables with $u_{1}, u_{2}$ and $u_{3}$ look quite similar to the case with no controls. Infected variable changes drastically-the number of infected population has dropped from the maximum point (Figure 5.3). The graphs of the Water $(W)$ and Dumpsite $(D)$ variables show similar results. The number of infected population gradually increases and reaches its maximum.

When the infectious group's population reaches its peak, $u_{1}$ and $u_{3}$ increase rapidly. 
After the peak time, $u_{1}$ and $u_{3}$ are steady, and $u_{2}$ increase gradually until time equals to 55 .
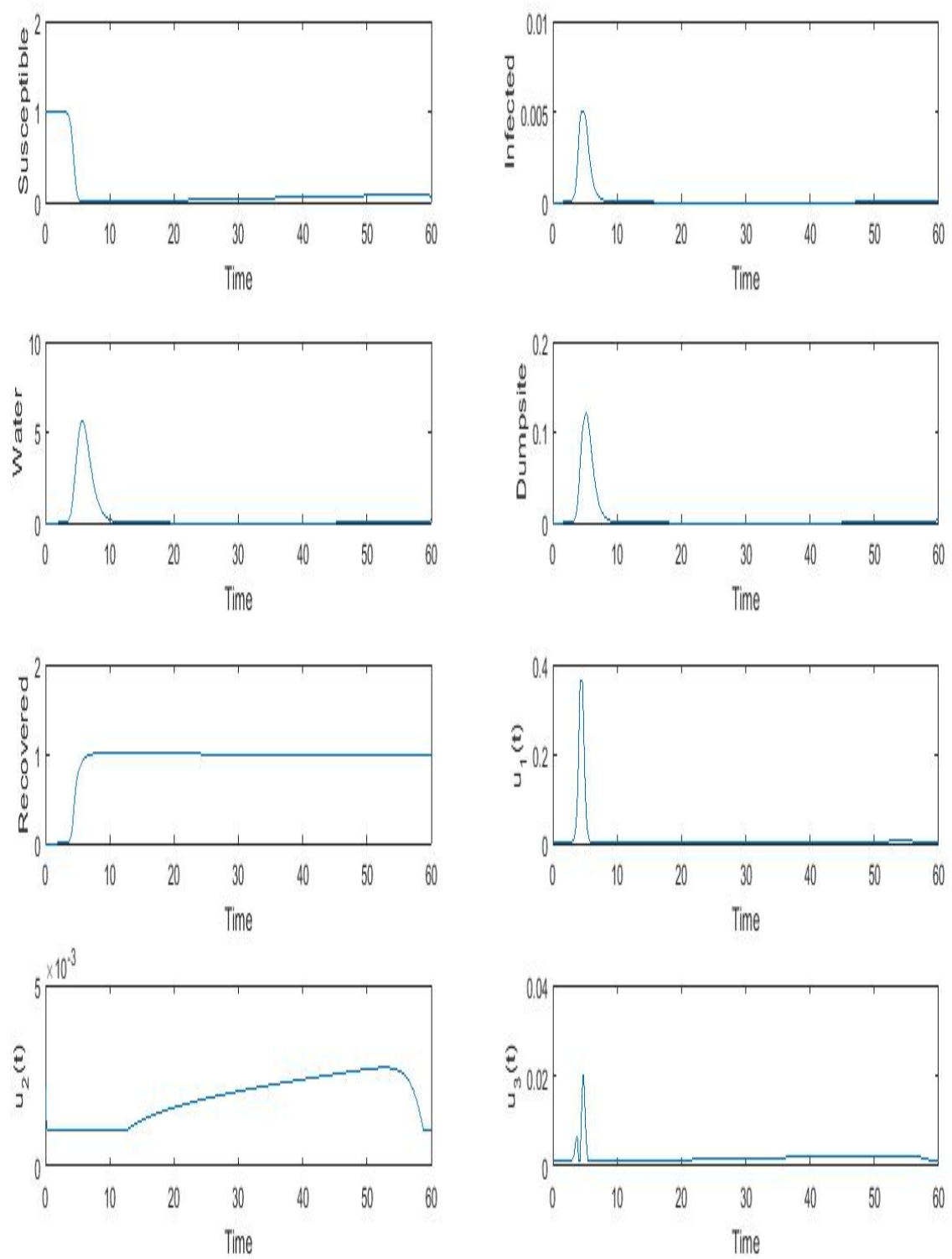

Figure 5.3: Case 1: Solutions of the SIWDR model with $u_{1}, u_{2}$ and $u_{3}$.

The peak of cholera epidemic decreases when controls that involve $u_{1}$ are applied (Figure 5.4). However, the peak time stays close to the peak time of the reference 
situation. Figure 5.5 shows that controls involving $u_{1}$ have the similar dynamic in infected compartment.

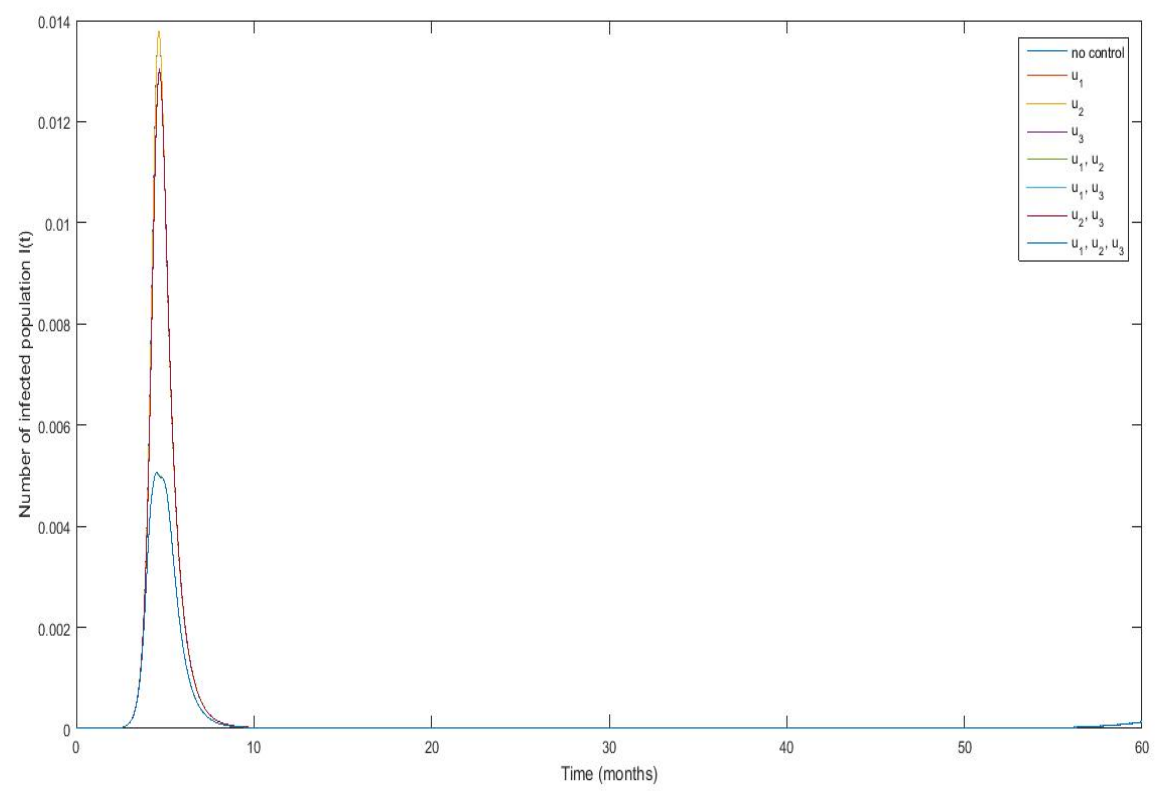

Figure 5.4: Case 1: The number of infected population in the eight different situations.

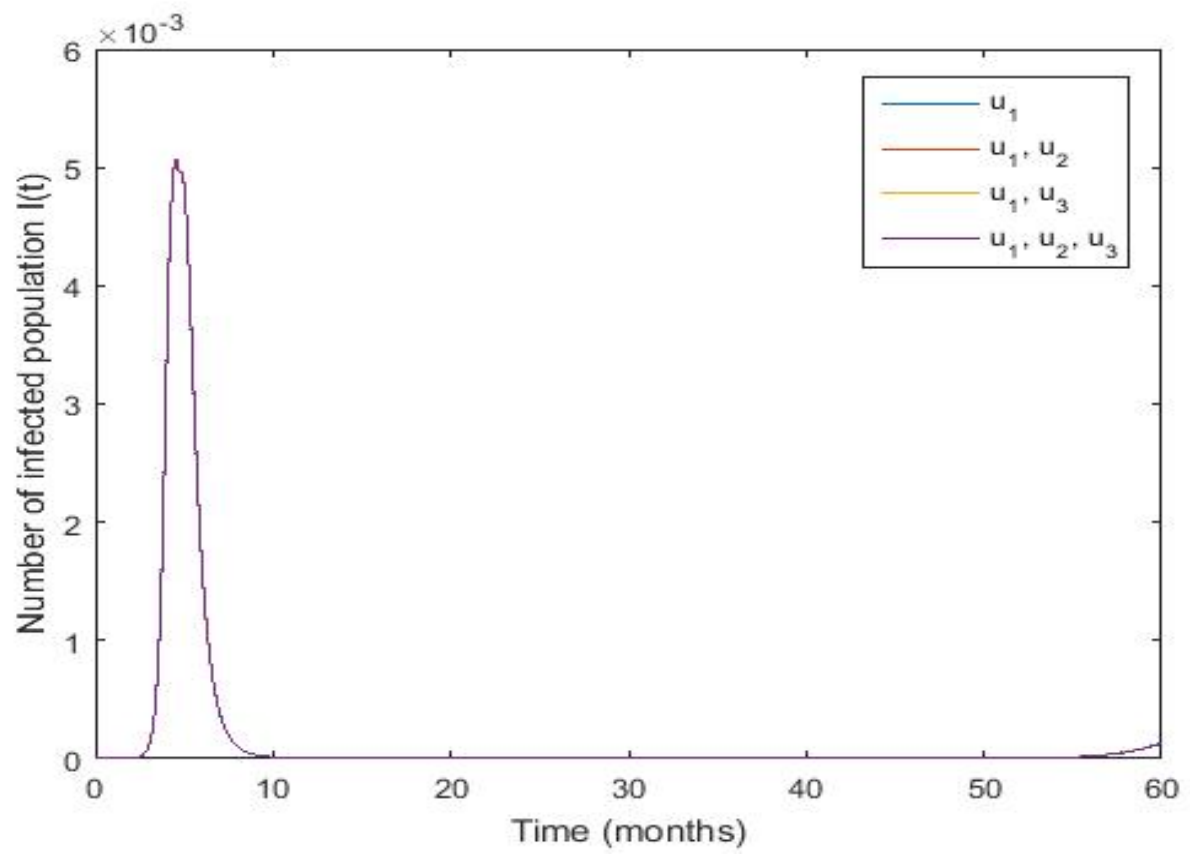

Figure 5.5: Case 1: The number of infected population in the four different situations that involve $u_{1}$. 
Weights of $u_{1}, u_{2}$ and $u_{3}$ affect the total number of infected population, and determine how to allocate budget to each service (See Table 5.2 below).

Table 5.2: Case 1: Sensitivity analysis of various control parameters.

\begin{tabular}{|c|c|c|}
\hline Case & Total number of infected population & $k_{I}$ \\
\hline $\mathrm{A}=3, \tau_{1}=1, \tau_{2}=1, \tau_{3}=1$ & 0.1758 & 0.00 \\
\hline $\mathrm{A}=3, \tau_{1}=1, \tau_{2}=1, \tau_{3}=2$ & 0.1758 & 0.00 \\
\hline $\mathrm{A}=3, \tau_{1}=1, \tau_{2}=1, \tau_{3}=3$ & 0.1758 & 0.00 \\
\hline $\mathrm{A}=3, \tau_{1}=1, \tau_{2}=2, \tau_{3}=1$ & 0.1759 & 0.06 \\
\hline $\mathrm{A}=3, \tau_{1}=1, \tau_{2}=2, \tau_{3}=2$ & 0.1759 & 0.06 \\
\hline $\mathrm{A}=3, \tau_{1}=1, \tau_{2}=2, \tau_{3}=3$ & 0.1759 & 0.06 \\
\hline $\mathrm{A}=3, \tau_{1}=1, \tau_{2}=3, \tau_{3}=1$ & 0.1759 & 0.06 \\
\hline $\mathrm{A}=3, \tau_{1}=1, \tau_{2}=3, \tau_{3}=2$ & 0.1760 & 0.11 \\
\hline $\mathrm{A}=3, \tau_{1}=1, \tau_{2}=3, \tau_{3}=3$ & 0.1760 & 0.11 \\
\hline $\mathrm{A}=3, \tau_{1}=2, \tau_{2}=1, \tau_{3}=1$ & 0.2426 & 38.00 \\
\hline $\mathrm{A}=3, \tau_{1}=2, \tau_{2}=1, \tau_{3}=2$ & 0.2429 & 38.17 \\
\hline $\mathrm{A}=3, \tau_{1}=2, \tau_{2}=1, \tau_{3}=3$ & 0.2429 & 38.17 \\
\hline $\mathrm{A}=3, \tau_{1}=2, \tau_{2}=2, \tau_{3}=1$ & 0.2428 & 38.11 \\
\hline $\mathrm{A}=3, \tau_{1}=2, \tau_{2}=2, \tau_{3}=2$ & 0.2430 & 38.23 \\
\hline $\mathrm{A}=3, \tau_{1}=2, \tau_{2}=2, \tau_{3}=3$ & 0.2431 & 38.28 \\
\hline $\mathrm{A}=3, \tau_{1}=2, \tau_{2}=3, \tau_{3}=1$ & 0.2428 & 38.11 \\
\hline $\mathrm{A}=3, \tau_{1}=2, \tau_{2}=3, \tau_{3}=2$ & 0.2430 & 38.23 \\
\hline $\mathrm{A}=3, \tau_{1}=2, \tau_{2}=3, \tau_{3}=3$ & 0.2431 & 38.28 \\
\hline $\mathrm{A}=3, \tau_{1}=3, \tau_{2}=1, \tau_{3}=1$ & 0.2680 & 41.07 \\
\hline $\mathrm{A}=3, \tau_{1}=3, \tau_{2}=1, \tau_{3}=2$ & 0.2691 & 53.06 \\
\hline $\mathrm{A}=3, \tau_{1}=3, \tau_{2}=1, \tau_{3}=3$ & 0.2694 & 53.25 \\
\hline $\mathrm{A}=3, \tau_{1}=3, \tau_{2}=2, \tau_{3}=1$ & 0.2681 & 52.50 \\
\hline $\mathrm{A}=3, \tau_{1}=3, \tau_{2}=2, \tau_{3}=2$ & 0.2692 & 53.14 \\
\hline $\mathrm{A}=3, \tau_{1}=3, \tau_{2}=2, \tau_{3}=3$ & 0.2696 & 53.33 \\
\hline $\mathrm{A}=3, \tau_{1}=3, \tau_{2}=3, \tau_{3}=1$ & 0.2681 & 52.51 \\
\hline $\mathrm{A}=3, \tau_{1}=3, \tau_{2}=3, \tau_{3}=2$ & 0.2692 & 53.15 \\
\hline $\mathrm{A}=3, \tau_{1}=3, \tau_{2}=3, \tau_{3}=3$ & 0.2696 & 53.34 \\
\hline $\mathrm{A}=2, \tau_{1}=1, \tau_{2}=1, \tau_{3}=1$ & 0.2176 & 23.77 \\
\hline $\mathrm{A}=1, \tau_{1}=1, \tau_{2}=1, \tau_{3}=1$ & 0.2696 & 53.34 \\
\hline $\mathrm{A}=0.1, \tau_{1}=1, \tau_{2}=1, \tau_{3}=1$ & 0.3205 & 82.33 \\
\hline $\mathrm{A}=3.1, \tau_{1}=1, \tau_{2}=1, \tau_{3}=1$ & 0.1732 & -1.45 \\
\hline $\mathrm{A}=3.2, \tau_{1}=1, \tau_{2}=1, \tau_{3}=1$ & 0.1709 & -2.78 \\
\hline
\end{tabular}

From the table 5.2, one can see that changing the weights of $u_{1}, u_{2}$ and $u_{3}$ results in change of the total number of infected population. These weights represent relative prices of technology in each service. 
For case $2\left(R_{0}<1\right.$; disease-free equilibrium), the total number of infected population is summarized in the table 5.3:

Table 5.3: Case 2: Comparison of number of infected population in the situation with no controls, and that with controls.

\begin{tabular}{|l|c|c|}
\hline Situation & Total number of infected population & $k_{I}$ \\
\hline 1. no controls & 0.1606 & 0.00 \\
\hline 2. $u_{1}$ & 0.1226 & -23.66 \\
\hline 3. $u_{2}$ & 0.1606 & 0.00 \\
\hline 4. $u_{3}$ & 0.1592 & -0.87 \\
\hline 5. $u_{1}, u_{2}$ & 0.1227 & -23.60 \\
\hline 6. $u_{1}, u_{3}$ & 0.1226 & -23.66 \\
\hline 7. $u_{2}, u_{3}$ & 0.1592 & -0.87 \\
\hline 8. $u_{1}, u_{2}, u_{3}$ & 0.1227 & -23.60 \\
\hline
\end{tabular}

The situation 2 with controls in DWS services $\left(u_{1}\right)$ and the situation 6 with controls in DWS and MSW services $\left(u_{1}\right.$ and $\left.u_{3}\right)$ result in the least number of infected population (-23.66\%). Also, $u_{1}$ or controls in DWS services is the most influential factor in reducing the number of infected population. This is seen in the situation 2 , 5,6 , and 8 where $u_{1}$ exists, and the total number of infected population is reduced in approximately the same amount. $u_{2}(0 \%)$ and $u_{3}(-0.87 \%)$ have little or no influence compared to the $u_{1}$. Figure 5.6 below shows the dynamic of all variables, $u_{1}, u_{2}$, and $u_{3}$ in the SIWDR model, in the situation 8.

The graphs of the Susceptible $(S)$, Infected $(I)$, and Recovered $(R)$ variables with $u_{1}, u_{2}$, and $u_{3}$ look quite similar to the case with no controls. Infected variable changes drastically-the number of infected population has dropped from the maximum point (Figure 5.6). The graphs of the Water $(W)$ and Dumpsite $(D)$ variables show similar results. The number of infected population gradually increases and reaches its maximum. 

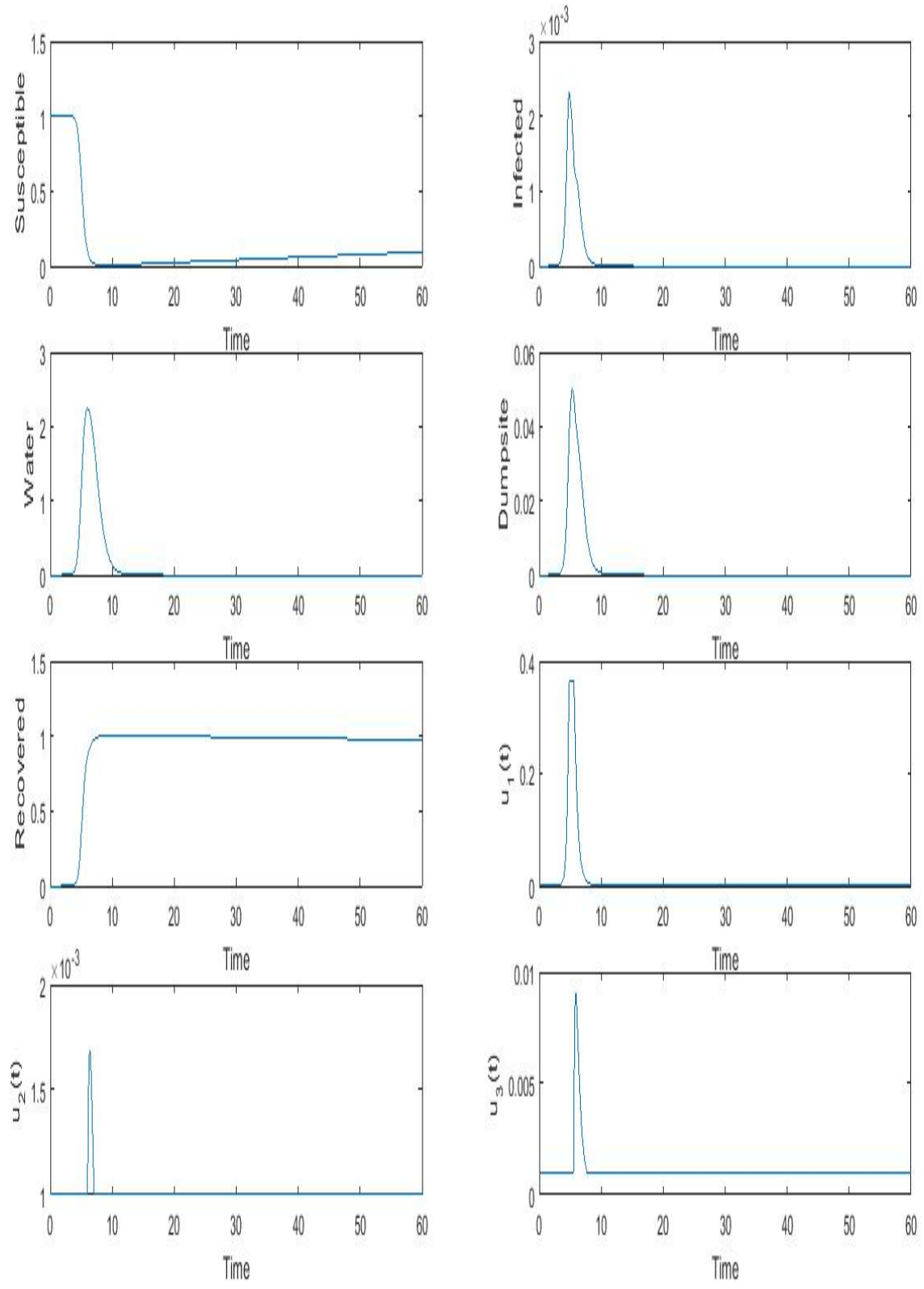

Figure 5.6: Case 2: Solutions of the SIWDR model with $u_{1}, u_{2}$ and $u_{3}$.

When the infectious group's population reaches its peak, $u_{1}, u_{2}$, and $u_{3}$ increase rapidly. After the peak time, $u_{1}, u_{2}$, and $u_{3}$ are steady.

The peak of cholera epidemic decreases when controls that involve $u_{1}$ are applied (Figure 5.7). However, the peak time stays close to the peak time of the reference 
situation. Figure 5.8 shows that controls involving $u_{1}$ have the similar dynamic in infected compartment.

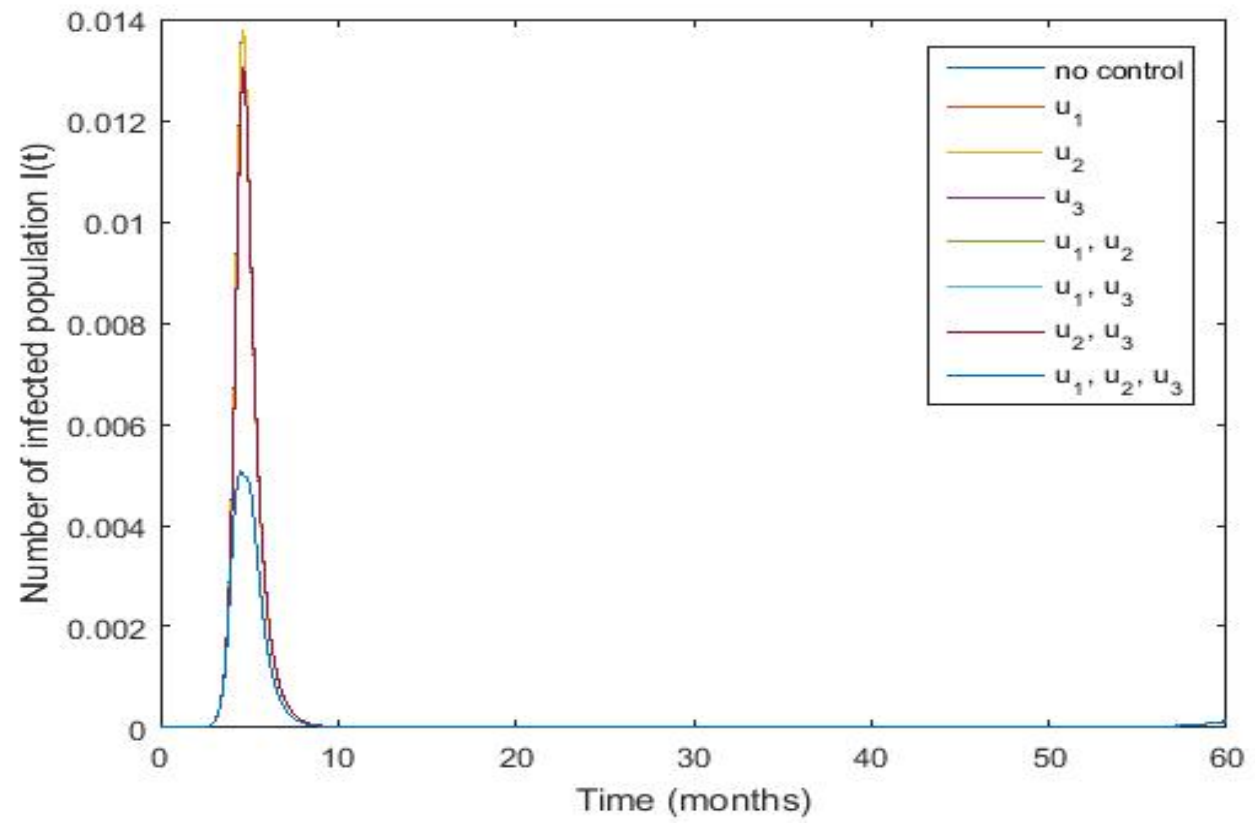

Figure 5.7: Case 2: The number of infected population in the eight different situations.

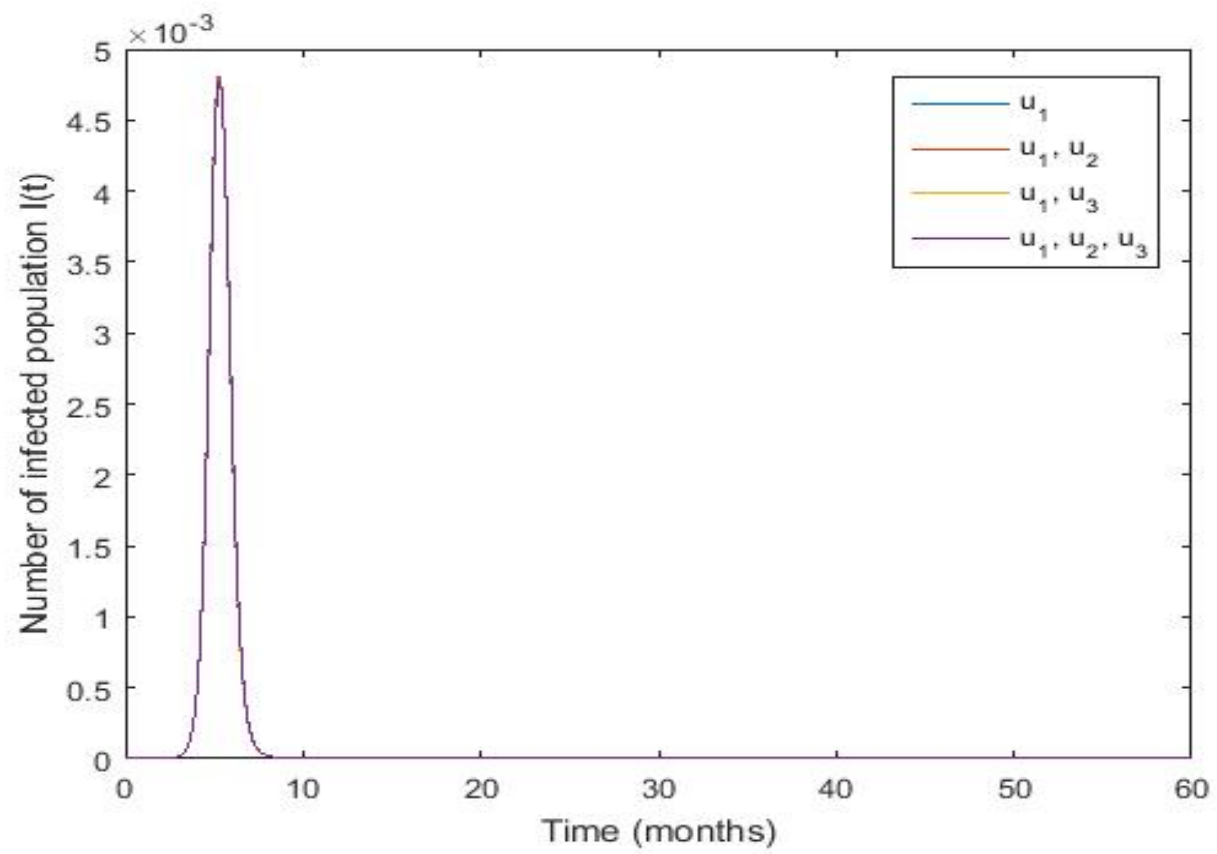

Figure 5.8: Case 2: The number of infected population in the four different situations that involve $u_{1}$. 
Weights of $u_{1}, u_{2}$, and $u_{3}$ affect the total number of infected population, and determine how to allocate budget to each service (See Table 5.4 below).

Table 5.4: Case 2: Sensitivity analysis of various control parameters.

\begin{tabular}{|c|c|c|}
\hline Case & Total number of infected population & $k_{I}$ \\
\hline $\mathrm{A}=3, \tau_{1}=1, \tau_{2}=1, \tau_{3}=1$ & 0.1227 & 0.00 \\
\hline $\mathrm{A}=3, \tau_{1}=1, \tau_{2}=1, \tau_{3}=2$ & 0.1227 & 0.00 \\
\hline $\mathrm{A}=3, \tau_{1}=1, \tau_{2}=1, \tau_{3}=3$ & 0.1227 & 0.00 \\
\hline $\mathrm{A}=3, \tau_{1}=1, \tau_{2}=2, \tau_{3}=1$ & 0.1227 & 0.00 \\
\hline $\mathrm{A}=3, \tau_{1}=1, \tau_{2}=2, \tau_{3}=2$ & 0.1227 & 0.00 \\
\hline $\mathrm{A}=3, \tau_{1}=1, \tau_{2}=2, \tau_{3}=3$ & 0.1227 & 0.00 \\
\hline $\mathrm{A}=3, \tau_{1}=1, \tau_{2}=3, \tau_{3}=1$ & 0.1227 & 0.00 \\
\hline $\mathrm{A}=3, \tau_{1}=1, \tau_{2}=3, \tau_{3}=2$ & 0.1227 & 0.00 \\
\hline $\mathrm{A}=3, \tau_{1}=1, \tau_{2}=3, \tau_{3}=3$ & 0.1227 & 0.00 \\
\hline $\mathrm{A}=3, \tau_{1}=2, \tau_{2}=1, \tau_{3}=1$ & 0.1404 & 14.43 \\
\hline $\mathrm{A}=3, \tau_{1}=2, \tau_{2}=1, \tau_{3}=2$ & 0.1406 & 14.59 \\
\hline $\mathrm{A}=3, \tau_{1}=2, \tau_{2}=1, \tau_{3}=3$ & 0.1407 & 14.67 \\
\hline $\mathrm{A}=3, \tau_{1}=2, \tau_{2}=2, \tau_{3}=1$ & 0.1404 & 14.43 \\
\hline $\mathrm{A}=3, \tau_{1}=2, \tau_{2}=2, \tau_{3}=2$ & 0.1406 & 14.59 \\
\hline $\mathrm{A}=3, \tau_{1}=2, \tau_{2}=2, \tau_{3}=3$ & 0.1407 & 14.67 \\
\hline $\mathrm{A}=3, \tau_{1}=2, \tau_{2}=3, \tau_{3}=1$ & 0.1404 & 14.43 \\
\hline $\mathrm{A}=3, \tau_{1}=2, \tau_{2}=3, \tau_{3}=2$ & 0.1406 & 14.59 \\
\hline $\mathrm{A}=3, \tau_{1}=2, \tau_{2}=3, \tau_{3}=3$ & 0.1407 & 14.67 \\
\hline $\mathrm{A}=3, \tau_{1}=3, \tau_{2}=1, \tau_{3}=1$ & 0.1466 & 19.48 \\
\hline $\mathrm{A}=3, \tau_{1}=3, \tau_{2}=1, \tau_{3}=2$ & 0.1469 & 19.72 \\
\hline $\mathrm{A}=3, \tau_{1}=3, \tau_{2}=1, \tau_{3}=3$ & 0.1471 & 19.89 \\
\hline $\mathrm{A}=3, \tau_{1}=3, \tau_{2}=2, \tau_{3}=1$ & 0.1466 & 19.48 \\
\hline $\mathrm{A}=3, \tau_{1}=3, \tau_{2}=2, \tau_{3}=2$ & 0.1469 & 19.72 \\
\hline $\mathrm{A}=3, \tau_{1}=3, \tau_{2}=2, \tau_{3}=3$ & 0.1471 & 19.89 \\
\hline $\mathrm{A}=3, \tau_{1}=3, \tau_{2}=3, \tau_{3}=1$ & 0.1466 & 19.48 \\
\hline $\mathrm{A}=3, \tau_{1}=3, \tau_{2}=3, \tau_{3}=2$ & 0.1469 & 19.72 \\
\hline $\mathrm{A}=3, \tau_{1}=3, \tau_{2}=3, \tau_{3}=3$ & 0.1471 & 19.89 \\
\hline $\mathrm{A}=2, \tau_{1}=1, \tau_{2}=1, \tau_{3}=1$ & 0.1344 & 9.54 \\
\hline $\mathrm{A}=1, \tau_{1}=1, \tau_{2}=1, \tau_{3}=1$ & 0.1471 & 19.89 \\
\hline $\mathrm{A}=0.1, \tau_{1}=1, \tau_{2}=1, \tau_{3}=1$ & 0.1592 & 29.75 \\
\hline $\mathrm{A}=3.1, \tau_{1}=1, \tau_{2}=1, \tau_{3}=1$ & 0.1215 & -0.98 \\
\hline $\mathrm{A}=3.2, \tau_{1}=1, \tau_{2}=1, \tau_{3}=1$ & 0.1204 & -1.87 \\
\hline
\end{tabular}

Without a budget limit, I choose the optimal solution obtained from numerical simulations (Figure 5.3 and 5.6).

The table below is interpreted as follows: (DWS, WST, MSW) represents monthly 
proportions of budget allocated to each service, DWS, WST, and MSW respectively.

Table 5.5: Case 1: Comparison of number of infected population using different strategies.

\begin{tabular}{|l|c|c|}
\hline Strategy & Total number of infected population & $k_{I}$ \\
\hline 1. optimal solution-baseline & 0.1758 & 0.00 \\
\hline 2. $(1,1,1)$-equal proportion & 0.2665 & 51.59 \\
\hline 3. $(1,1,2)$ & 0.3132 & 78.16 \\
\hline 4. $(1,2,1)$ & 0.3152 & 79.29 \\
\hline 5. $(1,2,2)$ & 0.3181 & 80.94 \\
\hline 6. $(2,1,1)$ & 0.3055 & 73.78 \\
\hline 7. $(2,1,2)$ & 0.3120 & 77.47 \\
\hline 8. $(2,2,1)$ & 0.3132 & 78.16 \\
\hline
\end{tabular}

Table 5.6: Case 2: Comparison of number of infected population using different strategies.

\begin{tabular}{|l|c|c|}
\hline Strategy & Total number of infected population & $k_{I}$ \\
\hline 1. optimal solution-baseline & 0.1227 & 0.00 \\
\hline 2. $(1,1,1)$-equal proportion & 0.1485 & 21.02 \\
\hline 3. $(1,1,2)$ & 0.3242 & 164.22 \\
\hline 4. $(1,2,1)$ & 0.3246 & 164.45 \\
\hline 5. $(1,2,2)$ & 0.3252 & 165.04 \\
\hline 6. $(2,1,1)$ & 0.3226 & 162.92 \\
\hline 7. $(2,1,2)$ & 0.3240 & 164.06 \\
\hline 8. $(2,2,1)$ & 0.3242 & 164.22 \\
\hline
\end{tabular}

For case 1, the optimal solution reduces the number of infected people $51.59 \%$ more than the strategy that allocates budget equally among WatSan services.

For case 2, the optimal solution reduces the number of infected people $21.02 \%$ more than the strategy that allocates budget equally among WatSan services.

Strategy 6 is the common strategy. Typically, the budget would be allocated to DWS in a higher proportion than to WST and MSW. However, this strategy is $73.78 \%$ less effective at reducing the number of infected people than the optimal strategy for 
case 1 , and $162.92 \%$ less effective at reducing the number of infected people than the optimal solution for case 2 .

There are no strategies in case 1 and 2 that can produce greater reductions in number of infected people than the optimal strategy (strategy 1). Thus, I choose the optimal strategy.

With a budget limit, I can change the bound of each control, and then find the optimal solution. In this case, I use the Forward-Backward Sweeping Method (FBSM). The upper bound of each control parameter $\left(u_{1}, u_{2}\right.$, and $\left.u_{3}\right)$ is set to be monthly budget, which is not necessary the same for each month. After setting the upper bounds, I find the optimal solution. Once the optimal solution is obtained, I check whether the sum of budget used for each parameter goes beyond the monthly budget. If it's not, the optimal solution is final. However, if the sum goes beyond the monthly budget, I need to lower the upper bound of each parameter for that particular month. Then, the FBSM is used again. Again, I check whether the sum of budget used for each parameter goes beyond the monthly budget. If it's not, the optimal solution is final. If that's the case, the FBSM is repeated until the optimal solution can be obtained, with the sum of budget used for each parameter lower than or equal to the monthly budget.

\subsection{Summary of optimal control for SIWDR model}

The goal of this study is to control the spread of enteric diarrheal disease (EDDs) using the SIWDR model. The study focuses on controls in DWS, WST, and MSW services and takes into account any constraints. This is to control the number of cholera infected population. I propose an appropriate cost index and show solutions of the SIWDR model. To minimize the number of infected population, I apply the Pontryagin's maximum principle. I use the numerical simulation to find the optimal controls. Two 
cases are considered here: Case 1 is when $R_{0}>1$; endemic equilibrium. Case 2 is when $R_{0}<1$; disease-free equilibrium. I also test 8 different situations within each case: situations with 1) no control, 2) $\left.\left.\left.u_{1}, 3\right) u_{2}, 4\right) u_{3}, 5\right) u_{1}$ and $\left.u_{2}, 6\right) u_{1}$ and $\left.u_{3}, 7\right) u_{2}$ and $u_{3}$, and 8) $u_{1}, u_{2}$, and $u_{3}$. Then, I compare the total number of infected population for each situation. I show sensitivity analysis of various control parameters. Finally, the budget allocation strategy used in WatSan services is determined and I compare the optimal solution obtained from numerical simulations with other allocation strategies. The result shows that the optimal solution reduces the number of infected population more than any other strategies. 


\section{Chapter 6}

\section{Conclusions and Future Research Directions}

Lack of WatSan services is an important problem in many developing countries. The problem affects the health of people, especially the poor. Lack of WatSan services increases the risk of WatSan related illness and subsequently the productivity of those affected. This problem disproportionately affects the poor. This research, thus, aims to increase access to WatSan services, thereby reducing health risks under conditions of budget constraint. Increasing access to WatSan services results from an increase in supply. However, this will also increase the inter-service demand. This includes sludge from DWS and WST services which is treated as MSW. At the same time leachate from MSW landfills is treated at WST facilities. Lastly, recycled wastewater is an output of the WST services, and can become an input to the DWS services.

Since pathogens reside and multiply in these byproducts-sludge, leachate, and recycled wastewater, they must be properly managed. To do so, the relationships between DWS, WST, and MSW services (i.e., WatSan services) must be understood. This research created tools that help us understand these relationships, as well as

precisely determine differences between demand and supply. It used a systems approach 
to understand and convert these relationships into matrices for easy analysis and making application easy.

Having tools to aid in understanding the whole water and sanitation system, this research used mathematical models to explore the link between WatSan services and the incidence of EDD. From the existing literature, a basic math model commonly used in the analysis of the spread of disease is the SIR (Susceptible-Infected-Recovered) model. The SIR model has been developed into many other models, including the SIWDR (Susceptible-Infected-Water-Dumpsite-Recovered) model. This research uses the SIWDR model because it can illustrate the spread of waterborne diseases (i.e., EDDs) with inadequate WatSan services.

The goal is to optimally reduce the spread of EDDs by improving WatSan services in the math model created earlier. I use optimal control theory to find best optimal strategies in improving WatSan services; thus optimally reduce the spread of EDDs.

\subsection{Findings and insights}

This dissertation can be summarized as follows:

1. Firstly, I used a systems approach to construct an integrated water and sanitation system (IWSS) model. This model captures deficits in the quantity of WatSan services in all regions of concern.

2. I proposed a nonlinear differential equation model called SIWDR (SusceptibleInfected-Water-Dumpsite-Recovered) model. The model links the status of WatSan services to the total cases of enteric diarrheal diseases (EDDs) in the service regions. 
3. Finally, I used optimal control theory to the SIWDR model. This is to annually allocate budget to the three (DWS, WST, and MSW) services; while minimizing the incidence of EDD over a specified planning horizon, with budget constraints.

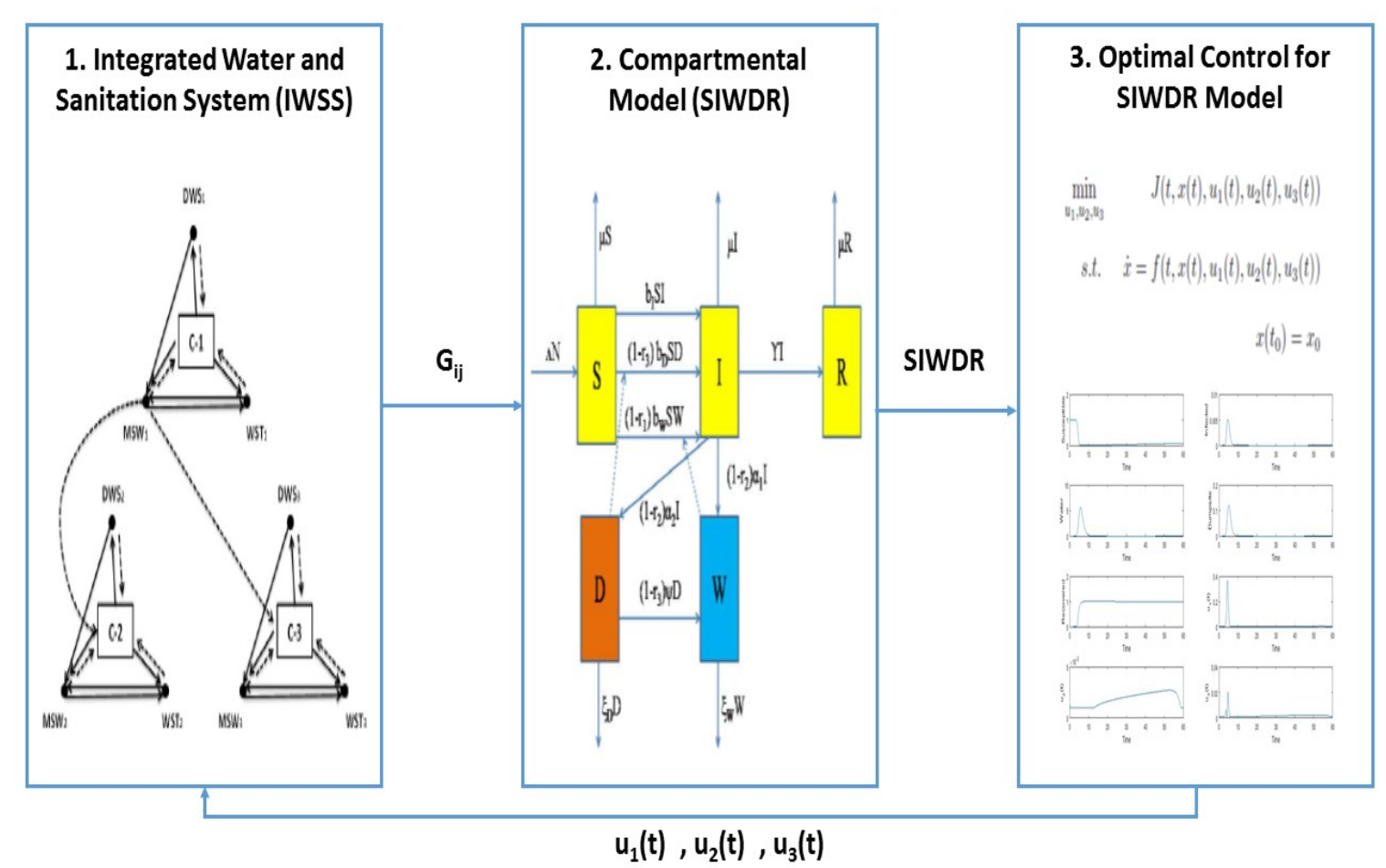

Figure 6.1: Models used in this study

These three steps might be used or developed further by other researchers. This might be a good starting point for improving WatSan services, and the related health of the people in communities.

The IWSS helps us understand relationships between DWS, WST, and MSW services in a particular community. Once the relationships are understood, WatSan services can be improved.

The SIWDR model is a mathematical model that illustrates how the status of WatSan services link to the spread of EDDs. I theoretically prove properties of the model, as well as the model's capability to reduce the total cases of EDDs-by putting 
controls in DWS, WST, and MSW services. This model can be used as a building block for improving WatSan services and health of the people in developing countries.

It can be noted that the added compartment $D$ includes but is not limited to dumpsites. Any surroundings where pathogens reside may apply. Regarding waterborne diseases, $D$ can refer to any natural habitats of pathogens besides water habitats. Improving $D$ or getting rid of $D$ means improving the aforementioned surroundings or getting rid of many routes of transmission of the diseases. Investing in improvement of $D$ can be done by means of a public health campaign, for instance. However, ways to improve $D$ depend on social context of the diseases.

Tools that I created in this study are useful for developing countries in improving health and WatSan services-especially the countries faced with disease outbreaks (e.g., cholera). These tools help with long-term planning and choosing the optimal strategies for improving WatSan services; thus optimally reduce the spread of EDDs.

\subsection{Future research directions}

This study discovers optimal strategies in improving WatSan services that optimally reduce the spread of EDDs. I utilize a systems approach, mathematical models, and optimal control theory. However, there are many more things that could be done in the future:

1. The model could be used in a specific case, with specific data. This way, values of each parameter could be directly measured, and precisely determined. An example of a specific case is a case study of cholera outbreak in refugee camps that do not have adequate WatSan services. 
2. The model can be made more realistic by not assuming that it reduces the spread of the diseases by $100 \%$. If that is the case, the mathematical model would be as follows:

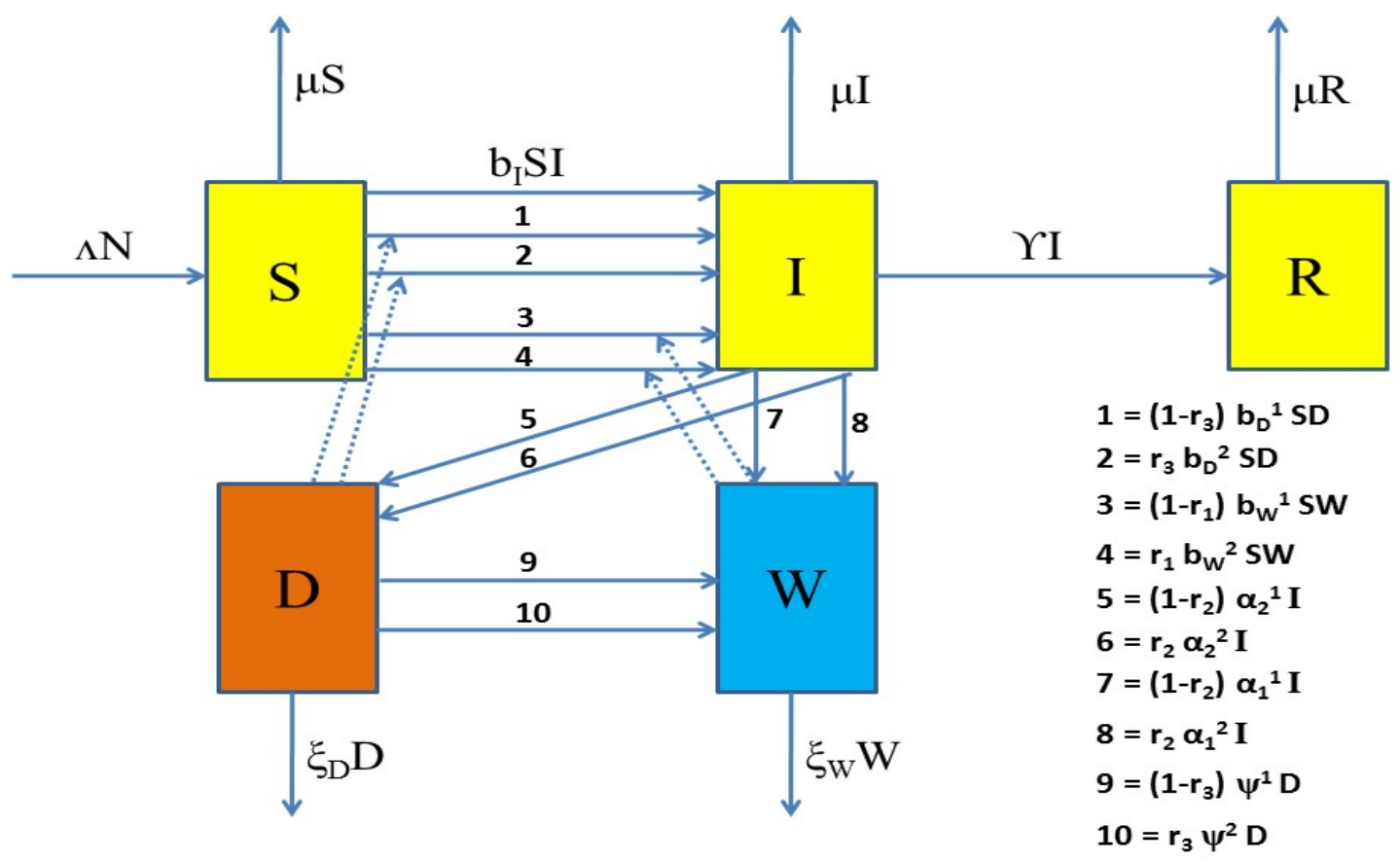

Figure 6.2: Flow diagram of the extended SIWDR model

Then, this model needs to be proved whether it fits all the theories used in this dissertation. This is to make sure that the extended SIWDR model works, can be used for further calculations, and can control the spread of WatSan related diseases. If control parameters are added to the model, the model will get more complicated and computationally intensive.

3. New objectives (e.g., economic, social, and environmental development) can be added to the model. This is because improvement of WatSan services can result in more economic, social, and environmental development, and not just better health effects. 
Appendix A 


\section{A.1 Assumptions (van den Driessche and Watmough $[74])$}

Assumptions to ensure the existence of equilibrium and ensure the model is well posed.

(A1) Assume $F_{i}^{0}(0, y)=0$ and $V_{i}^{0}(0, y)=0$ for all $y \geq 0$ and $i=1, \ldots, n$. All new infections are secondary infections are secondary infections arising from infected hosts; there is no imigration of individuals into the disease compartments.

(A2) Assume $F_{i}^{0}(x, y) \geq 0$ for all nonnegative $x$ and $y$ and $i=1, \ldots, n$. The function $F_{i}^{0}$ represents new infections and cannot be negative.

(A3) Assume $V_{i}^{0}(x, y) \leq 0$ wherever $x_{i}=0, i=1, \ldots, n$. Each compartment, $V_{i}^{0}$, represents a net outflow from compartment $i$ and must be negative (inflow only) whenever the compartment is empty.

(A4) Assume $\sum_{i=1}^{n} V_{i}^{0}(x, y) \geq 0$ for all nonnegative $x$ and $y$. This sum represents the total outflow from all infected compartments. Terms in the model leading to increases in $\sum_{i=1}^{n} x_{i}$ are assumed to represent secondary infections and therefore belong in $F^{0}$.

(A5) Assume the disease-free system $y^{\prime}=g(0, y)$ has a unique equilibrium that is asymptotically stable. That is, all solutions with initial conditions of the form $(0, y)$ approach a point $\left(0, y_{0}\right)$ as $t \rightarrow \infty$. We refer to this point as the disease-free equilibrium. 


\section{A.2 Theorem 5. (van den Driessche and Watmough $[74])$}

Consider the disease transmission model given by (4.2) and (4.3). The disease-free equilibrium of (4.2) and (4.3) is locally asymptotically stable if $R_{0}<1$, but unstable if $R_{0}>1$, where $R_{0}$ is defined as in section 4.1.

\section{A.3 Theorem 6. (van den Driessche and Watmough $[74])$}

If $A$ is a nonsingular $M-$ matrix and $\hat{f} \geq 0$, then the disease-free equilibrium of (4.6) and (4.7) is globally asymptotically stable. 
Appendix B 


\section{B.1 The range explored for parameters from table}

\section{3}

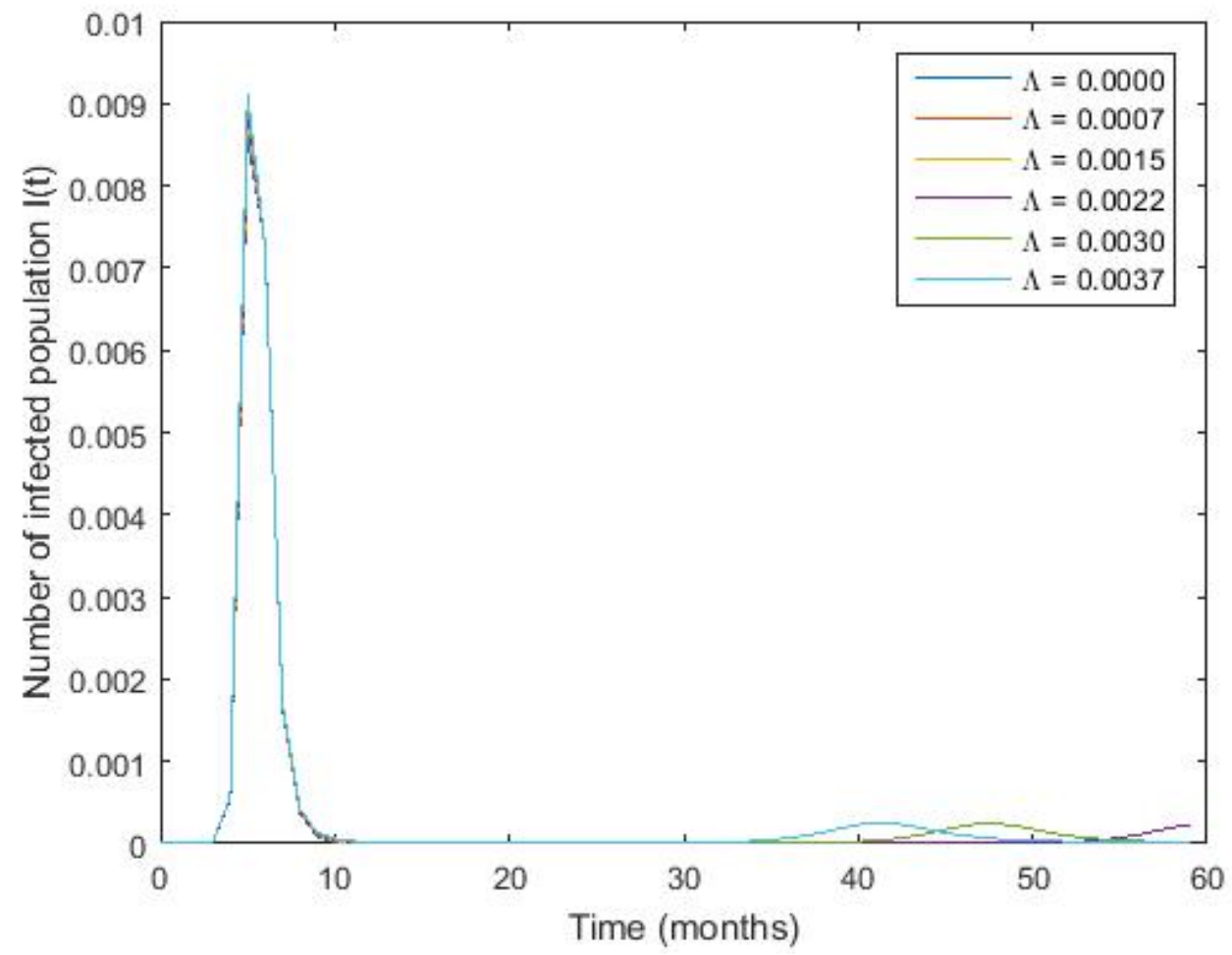

Figure B.1: Plot shows the number of infected people with various values of parameter, $\Lambda$.

Table B.1: Effect of $\Lambda$ on $R_{0}$ and $I$ at $t=60$.

\begin{tabular}{|c|c|c|c|}
\hline$\Lambda$ & $R_{0}$ & $I$ & Stable equilibrium \\
\hline 0.0000 & 0.0000 & 0.0000 & $\varepsilon_{0}$ \\
\hline 0.0007 & 0.8303 & 0.0000 & $\varepsilon_{0}$ \\
\hline 0.0015 & 1.7791 & 0.0000 & $\varepsilon_{1}$ \\
\hline 0.0022 & 2.6094 & 0.0082 & $\varepsilon_{1}$ \\
\hline 0.0030 & 3.5583 & 0.0000 & $\varepsilon_{1}$ \\
\hline 0.0037 & 4.3885 & 0.0000 & $\varepsilon_{1}$ \\
\hline
\end{tabular}




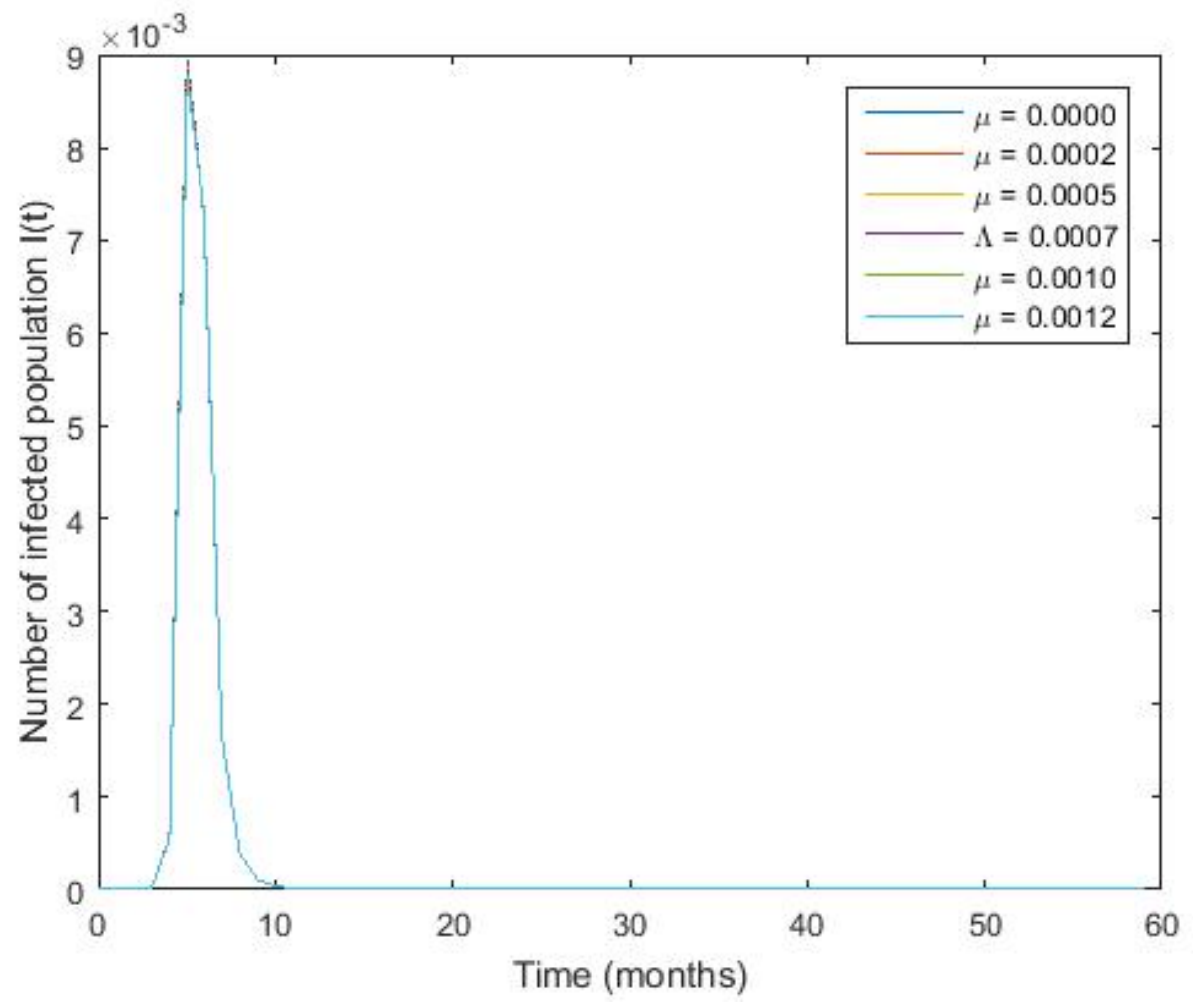

Figure B.2: Plot shows the number of infected people with various values of parameter, $\mu$.

Table B.2: Effect of $\mu$ on $R_{0}$ and $I$ at $t=60$.

\begin{tabular}{|c|c|c|c|}
\hline$\mu$ & $R_{0}$ & $I$ & Stable equilibrium \\
\hline 0.0000 & $\mathrm{n} / \mathrm{a}$ & 0.0002 & $\varepsilon_{1}$ \\
\hline 0.0002 & 6.8719 & 0.0002 & $\varepsilon_{1}$ \\
\hline 0.0005 & 2.7482 & 0.0001 & $\varepsilon_{1}$ \\
\hline 0.0007 & 1.9627 & 0.0001 & $\varepsilon_{1}$ \\
\hline 0.0010 & 1.3737 & 0.0001 & $\varepsilon_{1}$ \\
\hline 0.0012 & 1.1446 & 0.0001 & $\varepsilon_{1}$ \\
\hline
\end{tabular}




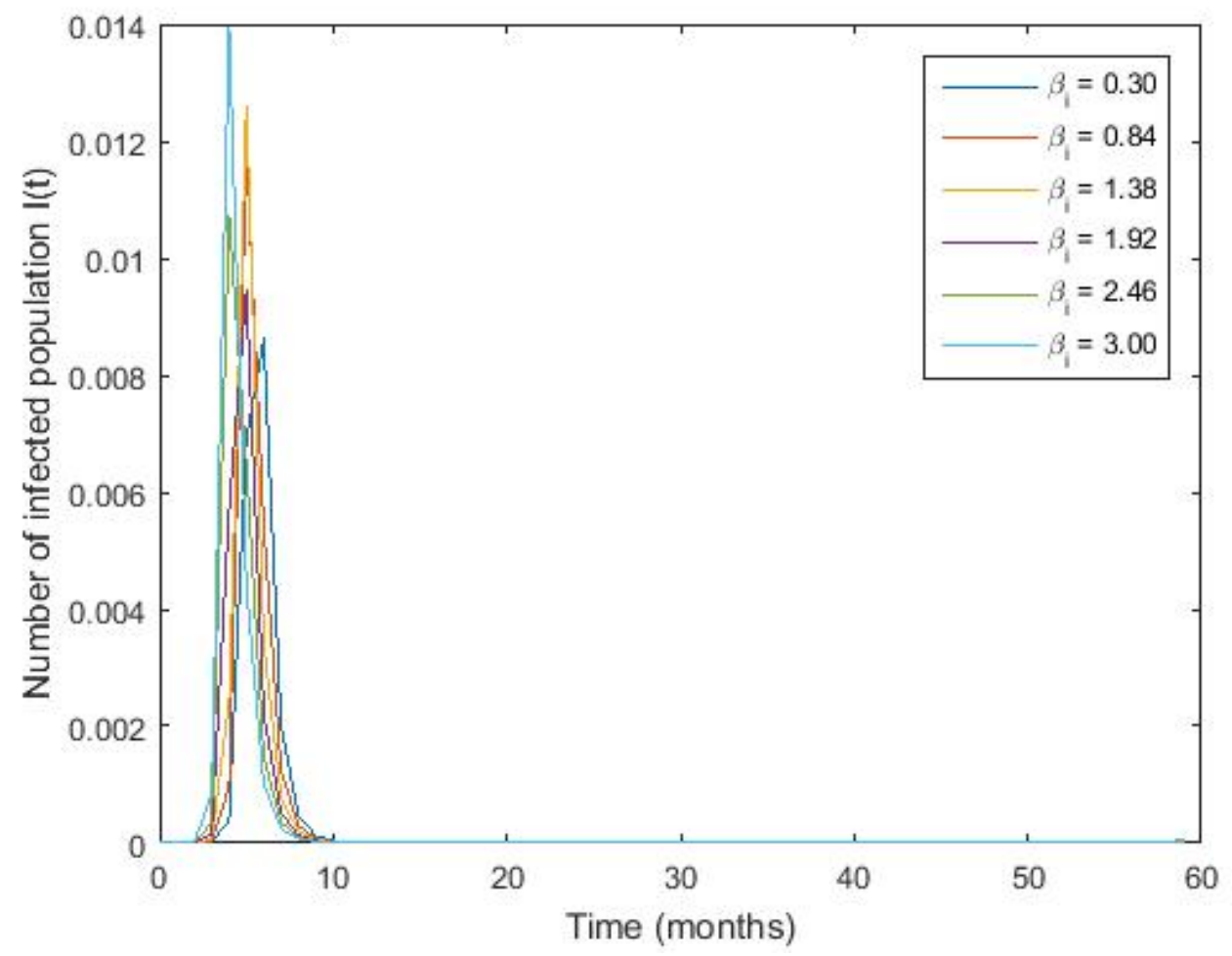

Figure B.3: Plot shows the number of infected people with various values of parameter, $\beta_{I}$.

Table B.3: Effect of $\beta_{I}$ on $R_{0}$ and $I$ at $t=60$.

\begin{tabular}{|c|c|c|c|}
\hline$\beta_{I}$ & $R_{0}$ & $I$ & Stable equilibrium \\
\hline 0.30 & 2.1225 & 0.0001 & $\varepsilon_{1}$ \\
\hline 0.84 & 2.1561 & 0.0002 & $\varepsilon_{1}$ \\
\hline 1.38 & 2.1896 & 0.0003 & $\varepsilon_{1}$ \\
\hline 1.92 & 2.2232 & 0.0006 & $\varepsilon_{1}$ \\
\hline 2.46 & 2.2567 & 0.0011 & $\varepsilon_{1}$ \\
\hline 3.00 & 2.2903 & 0.0018 & $\varepsilon_{1}$ \\
\hline
\end{tabular}




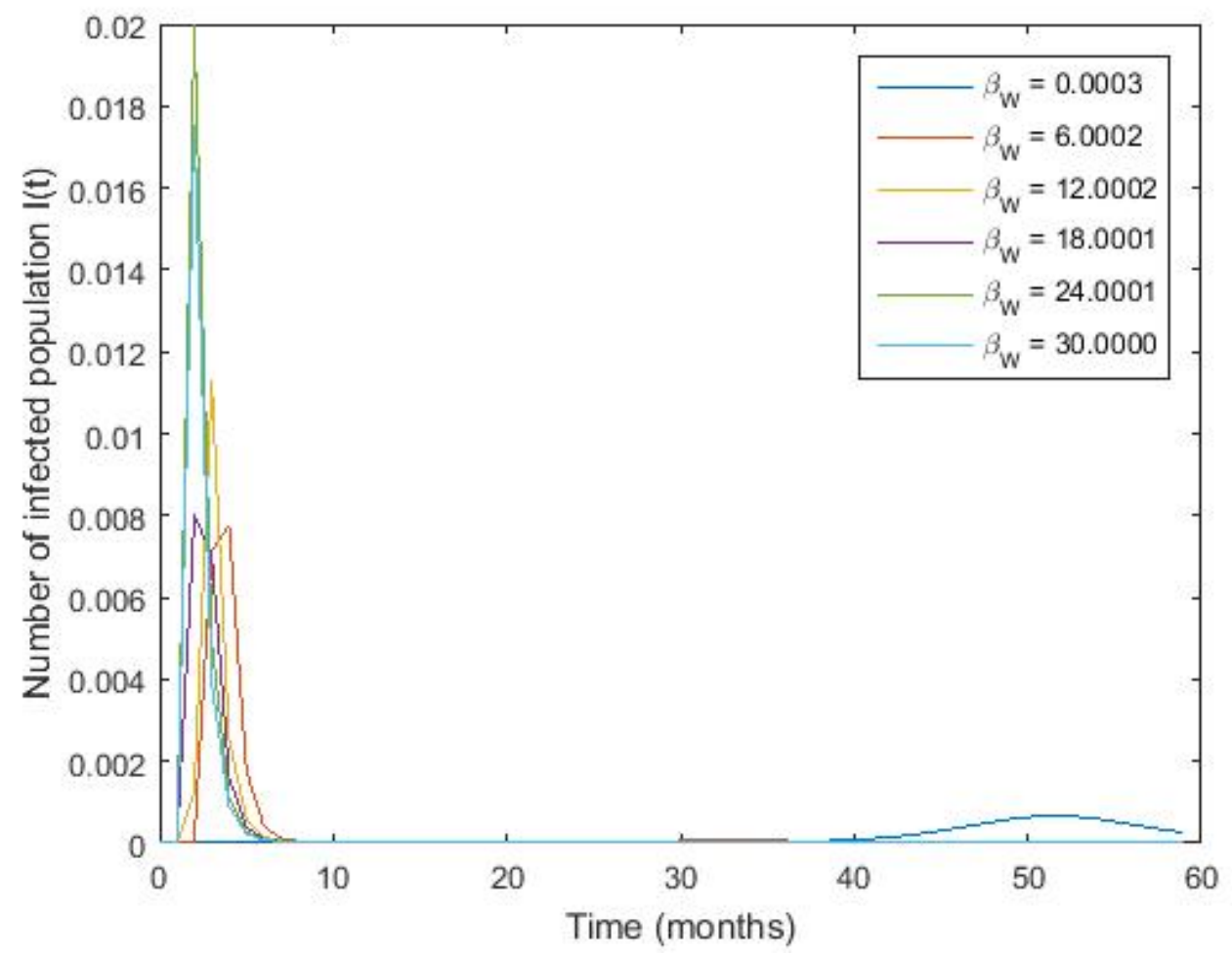

Figure B.4: Plot shows the number of infected people with various values of parameter, $\beta_{W}$.

Table B.4: Effect of $\beta_{W}$ on $R_{0}$ and $I$ at $t=60$.

\begin{tabular}{|c|c|c|c|}
\hline$\beta_{W}$ & $R_{0}$ & $I$ & Stable equilibrium \\
\hline 0.0003 & 0.1230 & 0.0091 & $\varepsilon_{0}$ \\
\hline 6.0002 & 6.1597 & 0.0017 & $\varepsilon_{1}$ \\
\hline 12.0002 & 12.1965 & 0.0012 & $\varepsilon_{1}$ \\
\hline 18.0001 & 18.2332 & 0.0012 & $\varepsilon_{1}$ \\
\hline 24.0001 & 24.2700 & 0.0012 & $\varepsilon_{1}$ \\
\hline 30.0000 & 30.3066 & 0.0012 & $\varepsilon_{1}$ \\
\hline
\end{tabular}




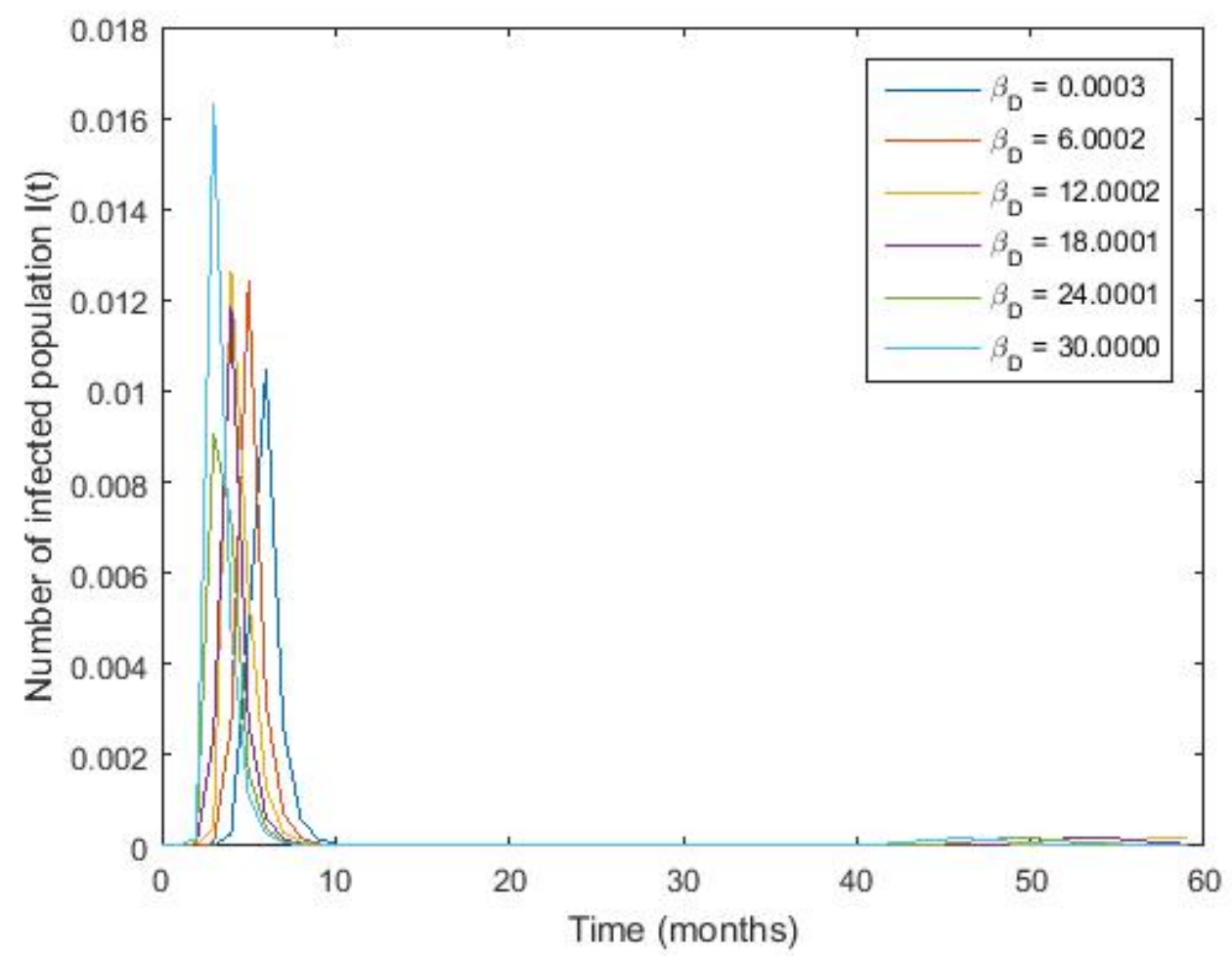

Figure B.5: Plot shows the number of infected people with various values of parameter, $\beta_{D}$.

Table B.5: Effect of $\beta_{D}$ on $R_{0}$ and $I$ at $t=60$.

\begin{tabular}{|c|c|c|c|}
\hline$\beta_{D}$ & $R_{0}$ & $I$ & Stable equilibrium \\
\hline 0.0003 & 2.0433 & 0.0000 & $\varepsilon_{1}$ \\
\hline 6.0002 & 2.1658 & 0.0013 & $\varepsilon_{1}$ \\
\hline 12.0002 & 2.2882 & 0.0071 & $\varepsilon_{1}$ \\
\hline 18.0001 & 2.4107 & 0.0016 & $\varepsilon_{1}$ \\
\hline 24.0001 & 2.5331 & 0.0004 & $\varepsilon_{1}$ \\
\hline 30.0000 & 2.6556 & 0.0003 & $\varepsilon_{1}$ \\
\hline
\end{tabular}




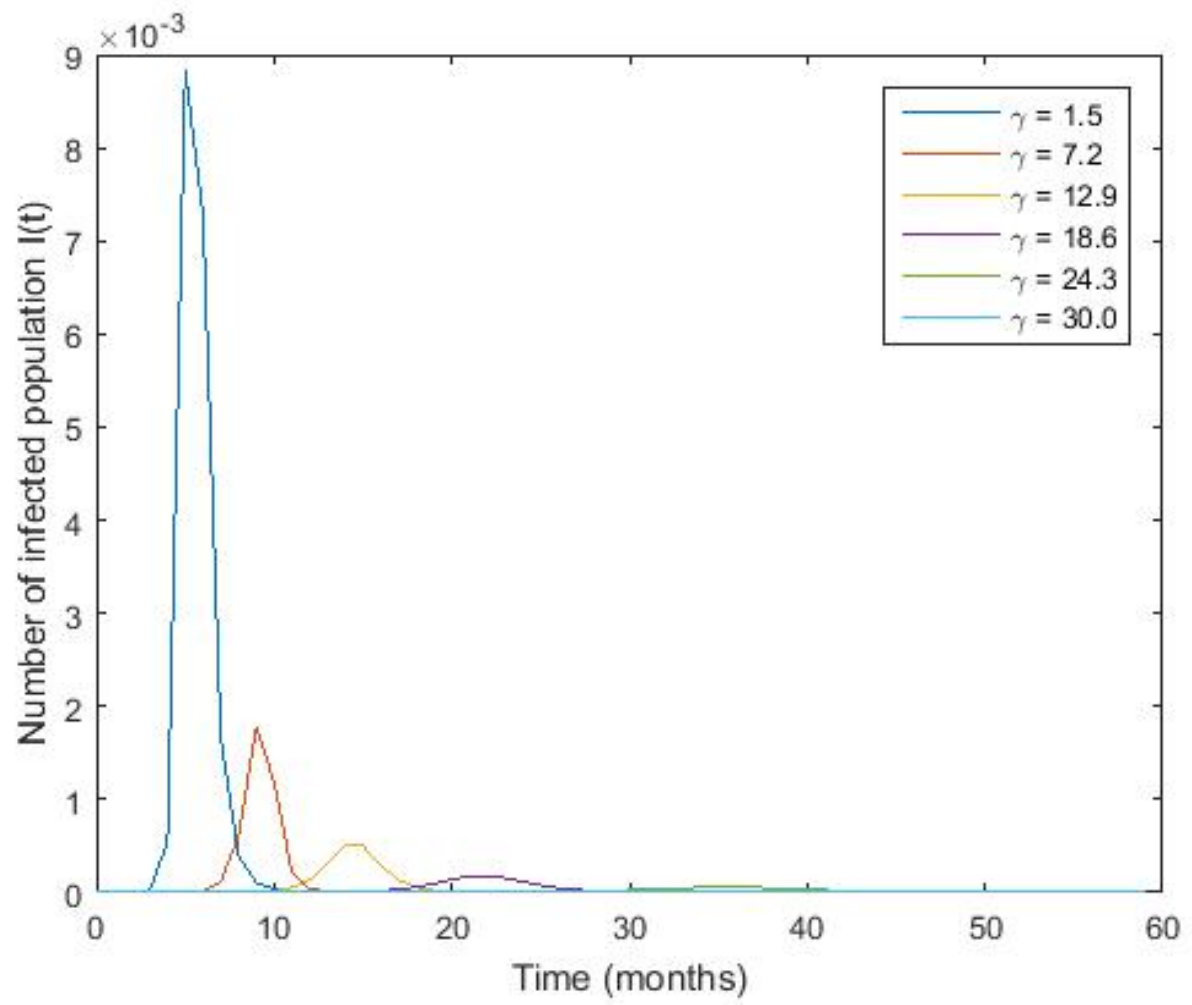

Figure B.6: Plot shows the number of infected people with various values of parameter, $\gamma$.

Table B.6: Effect of $\gamma$ on $R_{0}$ and $I$ at $t=60$.

\begin{tabular}{|c|c|c|c|}
\hline$\gamma$ & $R_{0}$ & $I$ & Stable equilibrium \\
\hline 1.5 & 2.1350 & 0.0001 & $\varepsilon_{1}$ \\
\hline 7.2 & 0.4449 & 0.0000 & $\varepsilon_{0}$ \\
\hline 12.9 & 0.2483 & 0.0000 & $\varepsilon_{0}$ \\
\hline 18.6 & 0.1722 & 0.0000 & $\varepsilon_{0}$ \\
\hline 24.3 & 0.1318 & 0.0000 & $\varepsilon_{0}$ \\
\hline 30.0 & 0.1068 & 0.0002 & $\varepsilon_{0}$ \\
\hline
\end{tabular}




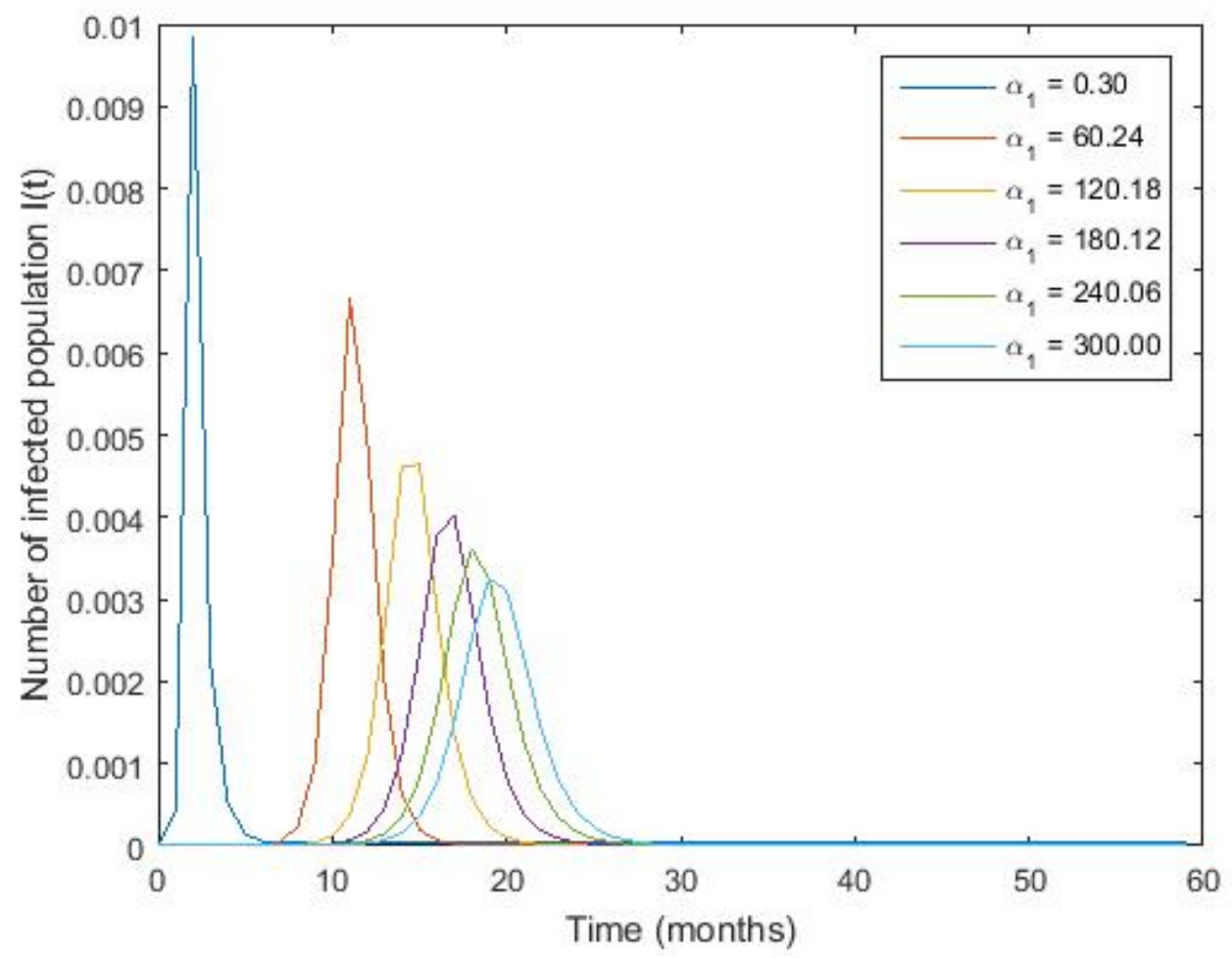

Figure B.7: Plot shows the number of infected people with various values of parameter, $\alpha_{1}$.

Table B.7: Effect of $\alpha_{1}$ on $R_{0}$ and $I$ at $t=60$.

\begin{tabular}{|c|c|c|c|}
\hline$\alpha_{1}$ & $R_{0}$ & $I$ & Stable equilibrium \\
\hline 0.30 & 66.0617 & 0.1 & $\varepsilon_{1}$ \\
\hline 60.24 & 0.4861 & 0.1 & $\varepsilon_{0}$ \\
\hline 120.18 & 0.3224 & 0.1 & $\varepsilon_{0}$ \\
\hline 180.12 & 0.2676 & 0.1 & $\varepsilon_{0}$ \\
\hline 240.06 & 0.2402 & 0.1 & $\varepsilon_{0}$ \\
\hline 300.00 & 0.2238 & 0.1 & $\varepsilon_{0}$ \\
\hline
\end{tabular}




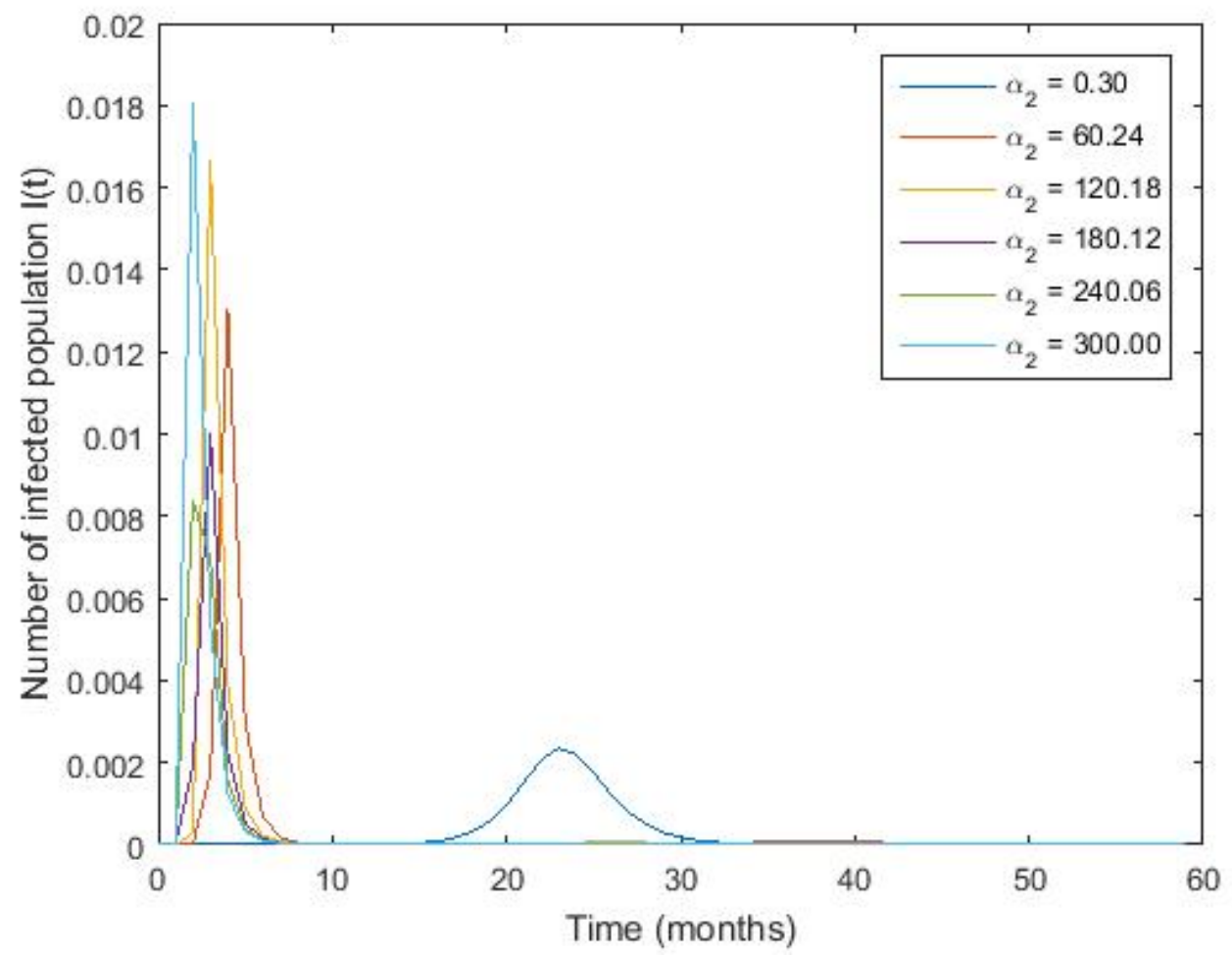

Figure B.8: Plot shows the number of infected people with various values of parameter, $\alpha_{2}$.

Table B.8: Effect of $\alpha_{2}$ on $R_{0}$ and $I$ at $t=60$.

\begin{tabular}{|c|c|c|c|}
\hline$\alpha_{2}$ & $R_{0}$ & $I$ & Stable equilibrium \\
\hline 0.30 & 0.1816 & 0.1 & $\varepsilon_{0}$ \\
\hline 60.24 & 4.9219 & 0.1 & $\varepsilon_{1}$ \\
\hline 120.18 & 9.6622 & 0.1 & $\varepsilon_{1}$ \\
\hline 180.12 & 14.4026 & 0.1 & $\varepsilon_{1}$ \\
\hline 240.06 & 19.1429 & 0.1 & $\varepsilon_{1}$ \\
\hline 300.00 & 23.8832 & 0.1 & $\varepsilon_{1}$ \\
\hline
\end{tabular}




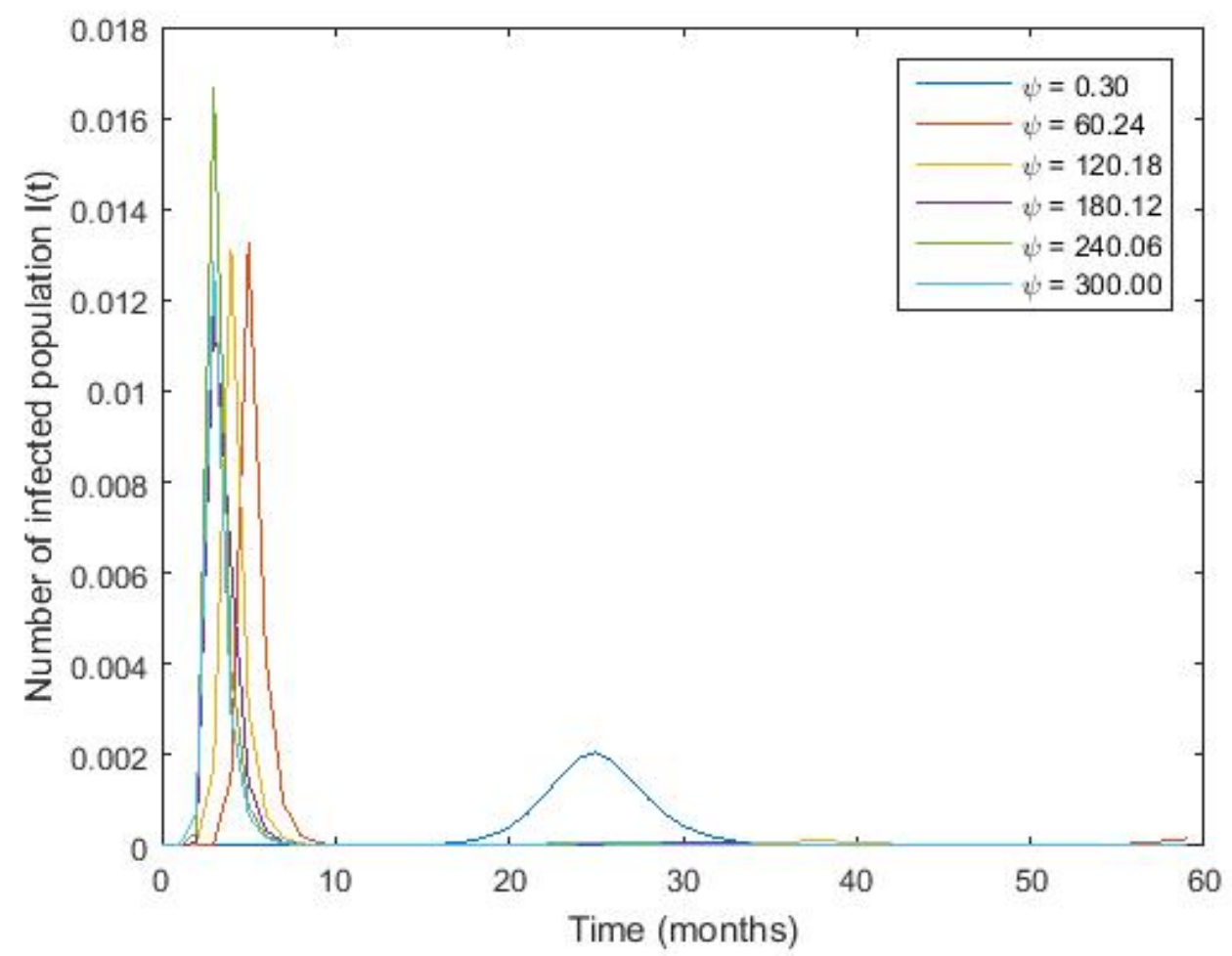

Figure B.9: Plot shows the number of infected people with various values of parameter, $\psi$.

Table B.9: Effect of $\psi$ on $R_{0}$ and $I$ at $t=60$.

\begin{tabular}{|c|c|c|c|}
\hline$\psi$ & $R_{0}$ & $I$ & Stable equilibrium \\
\hline 0.30 & 0.1697 & 0.1 & $\varepsilon_{0}$ \\
\hline 60.24 & 2.5399 & 0.1 & $\varepsilon_{1}$ \\
\hline 120.18 & 4.9100 & 0.1 & $\varepsilon_{1}$ \\
\hline 180.12 & 7.2802 & 0.1 & $\varepsilon_{1}$ \\
\hline 240.06 & 9.6504 & 0.1 & $\varepsilon_{1}$ \\
\hline 300.00 & 12.0205 & 0.1 & $\varepsilon_{1}$ \\
\hline
\end{tabular}




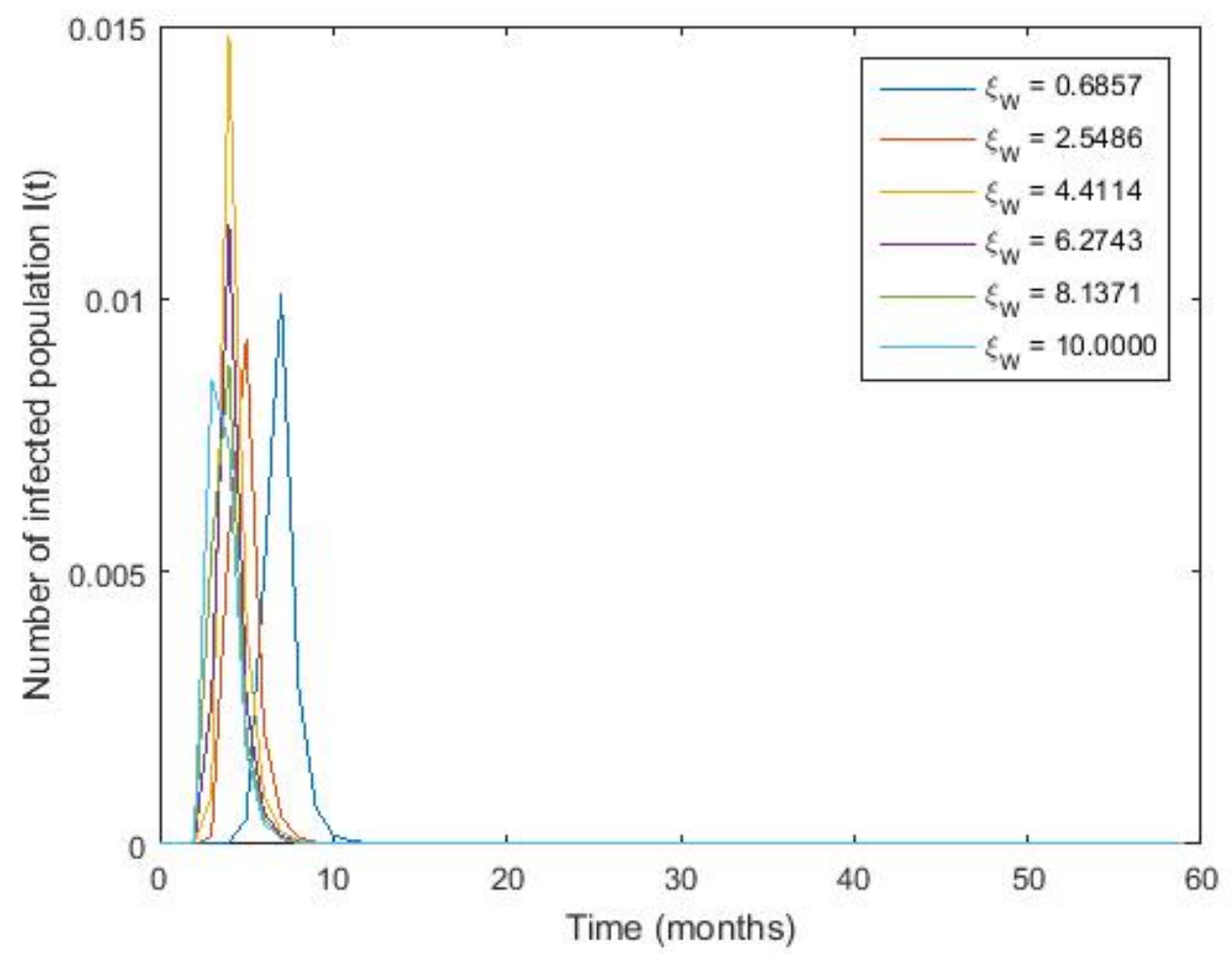

Figure B.10: Plot shows the number of infected people with various values of parameter, $\xi_{W}$.

Table B.10: Effect of $\xi_{W}$ on $R_{0}$ and $I$ at $t=60$.

\begin{tabular}{|c|c|c|c|}
\hline$\xi_{W}$ & $R_{0}$ & $I$ & Stable equilibrium \\
\hline 0.6857 & 2.1350 & 0.0002 & $\varepsilon_{1}$ \\
\hline 2.5486 & 2.1350 & 0.0003 & $\varepsilon_{1}$ \\
\hline 4.4114 & 2.1350 & 0.0004 & $\varepsilon_{1}$ \\
\hline 6.2743 & 2.1350 & 0.0006 & $\varepsilon_{1}$ \\
\hline 8.1371 & 2.1350 & 0.0008 & $\varepsilon_{1}$ \\
\hline 10.0000 & 2.1350 & 0.0010 & $\varepsilon_{1}$ \\
\hline
\end{tabular}

Varying $\xi_{W}$ does not change $R_{0}$. 


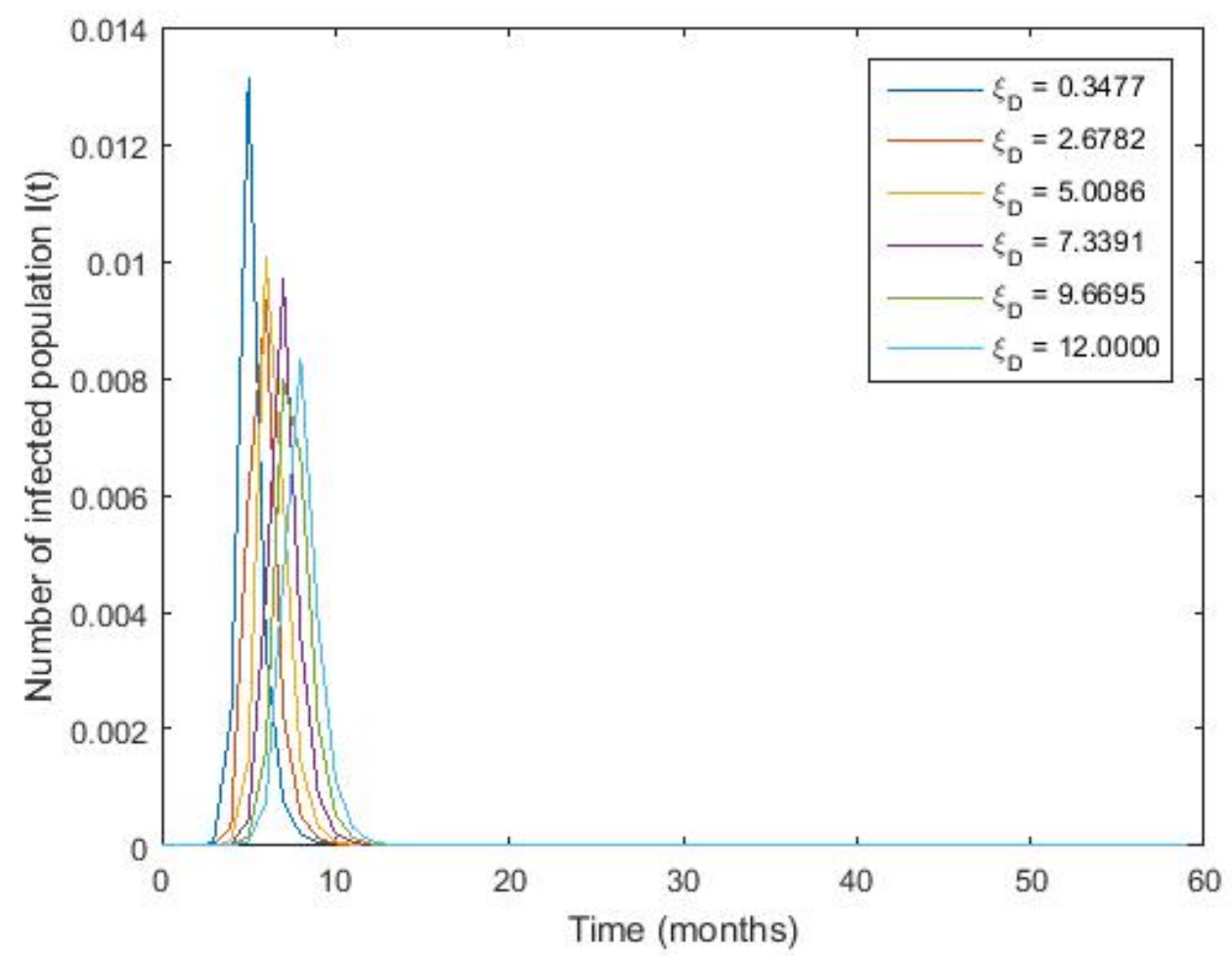

Figure B.11: Plot shows the number of infected people with various values of parameter, $\xi_{D}$.

Table B.11: Effect of $\xi_{D}$ on $R_{0}$ and $I$ at $t=60$.

\begin{tabular}{|c|c|c|c|}
\hline$\xi_{D}$ & $R_{0}$ & $I$ & Stable equilibrium \\
\hline 0.3477 & 11.5304 & 0.0012 & $\varepsilon_{1}$ \\
\hline 2.6782 & 1.6343 & 0.0000 & $\varepsilon_{1}$ \\
\hline 5.0086 & 0.9473 & 0.0000 & $\varepsilon_{0}$ \\
\hline 7.3391 & 0.6966 & 0.0000 & $\varepsilon_{0}$ \\
\hline 9.6695 & 0.5668 & 0.0000 & $\varepsilon_{0}$ \\
\hline 12.0000 & 0.4874 & 0.0000 & $\varepsilon_{0}$ \\
\hline
\end{tabular}


Appendix C 


\section{C.1 Matlab code: Forward-Backward Sweep Method}

\section{Matlab Code}

1 function $y=\operatorname{SIWDR}($ Lambda, mu, beta_i , beta_w, beta_d, gamma, alpha_1, alpha_2, psi, xi_w , xi_d, r1 , r2 , r3 , tau1, tau2, tau3, S0, $\mathrm{I0}, \mathrm{W} 0, \mathrm{D} 0, \mathrm{R} 0, \mathrm{~A}, \mathrm{~T})$

3 test $=-1$

4

${ }_{5}$ delta $=0.001 ;$

${ }_{6} \mathrm{M}=1000$;

$7 \mathrm{t}=$ linspace $(0, \mathrm{~T}, \mathrm{M}+1)$;

$8 \mathrm{~h}=\mathrm{T} / \mathrm{M}$;

${ }_{9} \mathrm{~h} 2=\mathrm{h} / 2$;

10

$11 \mathrm{~S}=\mathrm{zeros}(1, \mathrm{M}+1)$;

$12 \mathrm{I}=\mathrm{zeros}(1, \mathrm{M}+1)$;

${ }_{13} \mathrm{~W} \models \operatorname{zeros}(1, \mathrm{M}+1)$;

$14 \mathrm{D}=\operatorname{zeros}(1, \mathrm{M}+1)$;

$15 \mathrm{R}=\mathrm{zeros}(1, \mathrm{M}+1)$;

${ }_{16} \quad \mathrm{~S}(1)=\mathrm{S} 0$;

${ }_{17} \mathrm{I}(1)=\mathrm{I} 0$;

${ }_{18} \mathrm{~W}(1)=\mathrm{W} 0$;

$19 \mathrm{D}(1)=\mathrm{D} 0$;

${ }_{20} \quad \mathrm{R}(1)=\mathrm{R} 0$;

21 


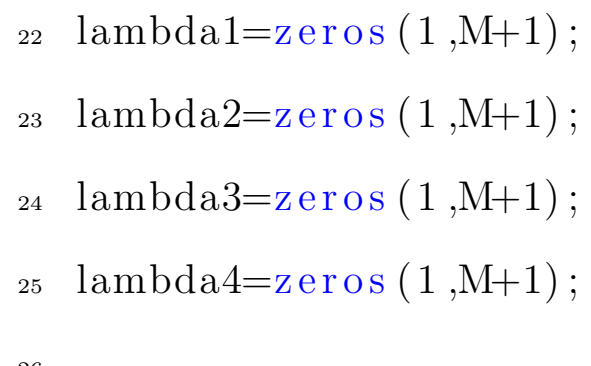

26

27 


$$
\begin{aligned}
& 49 \text { for } \mathrm{i}=1: \mathrm{M} \\
& { }_{50} \quad \mathrm{~m} 11=\text { Lambda }_{-}(1-\mathrm{r} 1) * \text { beta }_{-} \mathrm{W} * \mathrm{~S}(\mathrm{i}) * \mathrm{~W}(\mathrm{i})-(1-\mathrm{r} 3) * \text { bet a } \_\mathrm{d} * \mathrm{~S}( \\
& \text { i ) } * \mathrm{D}(\text { i })-\text { beta_i } * \mathrm{~S}(\text { i }) * \mathrm{I}(\text { i })-\mathrm{mu} * \mathrm{~S}(\text { i }) \text {; } \\
& 51 \\
& \mathrm{~m} 12=(1-\mathrm{r} 1-\mathrm{u}(\mathrm{i})) * \mathrm{beta}_{-} \mathrm{W} * \mathrm{~S}(\mathrm{i}) * \mathrm{~W}(\mathrm{i})+(1-\mathrm{r} 3-\mathrm{w}(\mathrm{i})) * \text { beta_d } \\
& * \mathrm{~S}(\mathrm{i}) * \mathrm{D}(\mathrm{i})+\text { beta_i }_{-} * \mathrm{~S}(\mathrm{i}) * \mathrm{I}(\mathrm{i})-\operatorname{gamma} * \mathrm{I}(\mathrm{i})-\mathrm{mu} * \mathrm{I}(\mathrm{i}) \text {; } \\
& \mathrm{m} 13=\mathrm{xi}_{-} \mathrm{w} *(1-\mathrm{r} 2-\mathrm{v}(\mathrm{i})) * \mathrm{I}(\mathrm{i})+((1-\mathrm{r} 3-\mathrm{w}(\mathrm{i})) * \mathrm{psi} * \text { alpha_ } 2 * \\
& \left.\mathrm{xi} \mathrm{i}_{-} \mathrm{w} /\left(\text { alpha_1 } * \mathrm{xi}_{-} \mathrm{d}\right)\right) * \mathrm{D}(\mathrm{i})-\mathrm{xi} \mathrm{w}_{-} * \mathrm{~W}(\mathrm{i}) \text {; }
\end{aligned}
$$

$\mathrm{m} 31=$ Lambda $_{-}(1-\mathrm{r} 1) *$ beta $_{-} \mathrm{W} *(\mathrm{~S}(\mathrm{i})+\mathrm{h} 2 * \mathrm{~m} 21) *(\mathrm{~W}(\mathrm{i})+\mathrm{h} 2 * \mathrm{~m} 23$ )$-(1-\mathrm{r} 3) * \mathrm{beta}_{-} \mathrm{d} *(\mathrm{~S}(\mathrm{i})+\mathrm{h} 2 * \mathrm{~m} 21) *(\mathrm{D}(\mathrm{i})+\mathrm{h} 2 * \mathrm{~m} 24)-\mathrm{bet}_{-} \mathrm{i}$ $*(\mathrm{~S}(\mathrm{i})+\mathrm{h} 2 * \mathrm{~m} 21) *(\mathrm{I}(\mathrm{i})+\mathrm{h} 2 * \mathrm{~m} 22)-\mathrm{mu} *(\mathrm{~S}(\mathrm{i})+\mathrm{h} 2 * \mathrm{~m} 21)$; 


$$
\mathrm{m} 32=(1-\mathrm{r} 1-0.5 *(\mathrm{u}(\mathrm{i})+\mathrm{u}(\mathrm{i}+1))) * \text { beta }_{-} \mathrm{w} *(\mathrm{~S}(\mathrm{i})+\mathrm{h} 2 * \mathrm{~m} 21) *(
$$

$\mathrm{W}(\mathrm{i})+\mathrm{h} 2 * \mathrm{~m} 23)+(1-\mathrm{r} 3-0.5 *(\mathrm{w}(\mathrm{i})+\mathrm{w}(\mathrm{i}+1))) * \mathrm{beta}_{-} \mathrm{d} *(\mathrm{~S}(\mathrm{i})$ $+\mathrm{h} 2 * \mathrm{~m} 21) *(\mathrm{D}(\mathrm{i})+\mathrm{h} 2 * \mathrm{~m} 24)+$ bet a $_{-} \mathrm{i} *(\mathrm{~S}(\mathrm{i})+\mathrm{h} 2 * \mathrm{~m} 21) *(\mathrm{I}(\mathrm{i})+$ $\mathrm{h} 2 * \mathrm{~m} 22)$-gamma $*(\mathrm{I}(\mathrm{i})+\mathrm{h} 2 * \mathrm{~m} 22)-\mathrm{mu} *(\mathrm{I}(\mathrm{i})+\mathrm{h} 2 * \mathrm{~m} 22)$; $\mathrm{m} 33=\mathrm{xi}-\mathrm{w} *(1-\mathrm{r} 2-0.5 *(\mathrm{v}(\mathrm{i})+\mathrm{v}(\mathrm{i}+1))) *(\mathrm{I}(\mathrm{i})+\mathrm{h} 2 * \mathrm{~m} 22)$ $+\left((1-\mathrm{r} 3-0.5 *(\mathrm{w}(\mathrm{i})+\mathrm{w}(\mathrm{i}+1))) * \mathrm{psi} * \mathrm{alph}_{-} 2 * \mathrm{xi} \mathrm{w}_{-} /(\right.$ alpha_ $\left.\left.1 * \mathrm{xi}_{-} \mathrm{d}\right)\right) *(\mathrm{D}(\mathrm{i})+\mathrm{h} 2 * \mathrm{~m} 24)-\mathrm{xi} \mathrm{I}_{\mathrm{w}} *(\mathrm{~W}(\mathrm{i})+\mathrm{h} 2 * \mathrm{~m} 23)$; $\mathrm{m} 34=\mathrm{xi}_{-} \mathrm{d} *(1-\mathrm{r} 2-0.5 *(\mathrm{v}(\mathrm{i})+\mathrm{v}(\mathrm{i}+1))) *(\mathrm{I}(\mathrm{i})+\mathrm{h} 2 * \mathrm{~m} 22)-($ $\left.\mathrm{xi} \mathrm{i}_{-} \mathrm{d} *(\mathrm{D}(\mathrm{i})+\mathrm{h} 2 * \mathrm{~m} 24)\right)$; $\mathrm{m} 41=$ Lambda $-(1-\mathrm{r} 1) *$ beta $_{-} \mathrm{W} *(\mathrm{~S}(\mathrm{i})+\mathrm{h} 2 * \mathrm{~m} 31) *(\mathrm{~W}(\mathrm{i})+\mathrm{h} 2 * \mathrm{~m} 33$ )$-(1-\mathrm{r} 3) *$ beta_d $*(\mathrm{~S}(\mathrm{i})+\mathrm{h} 2 * \mathrm{~m} 31) *(\mathrm{D}(\mathrm{i})+\mathrm{h} 2 * \mathrm{~m} 34)-\mathrm{bet}_{-} \mathrm{i}$ $*(\mathrm{~S}(\mathrm{i})+\mathrm{h} 2 * \mathrm{~m} 31) *(\mathrm{I}(\mathrm{i})+\mathrm{h} 2 * \mathrm{~m} 32)-\mathrm{mu} *(\mathrm{~S}(\mathrm{i})+\mathrm{h} 2 * \mathrm{~m} 31)$; $\mathrm{m} 42=(1-\mathrm{r} 1-\mathrm{u}(\mathrm{i}+1)) * \operatorname{beta}_{-} \mathrm{W} *(\mathrm{~S}(\mathrm{i})+\mathrm{h} 2 * \mathrm{~m} 31) *(\mathrm{~W}(\mathrm{i})+\mathrm{h} 2 * \mathrm{~m} 33$ )$+(1-\mathrm{r} 3-(\mathrm{w}(\mathrm{i}+1))) * \mathrm{beta}_{-} \mathrm{d} *(\mathrm{~S}(\mathrm{i})+\mathrm{h} 2 * \mathrm{~m} 31) *(\mathrm{D}(\mathrm{i})+\mathrm{h} 2 *$ $\mathrm{m} 34)+$ beta_i $*(\mathrm{~S}(\mathrm{i})+\mathrm{h} 2 * \mathrm{~m} 31) *(\mathrm{I}(\mathrm{i})+\mathrm{h} 2 * \mathrm{~m} 32)-\operatorname{gamma} *(\mathrm{I}$ ( i )$+\mathrm{h} 2 * \mathrm{~m} 32)-\mathrm{mu} *(\mathrm{I}(\mathrm{i})+\mathrm{h} 2 * \mathrm{~m} 32) ;$ $\mathrm{m} 43=\mathrm{xi}_{-} \mathrm{W} *(1-\mathrm{r} 2-\mathrm{v}(\mathrm{i}+1)) *(\mathrm{I}(\mathrm{i})+\mathrm{h} 2 * \mathrm{~m} 32)+((1-\mathrm{r} 3-\mathrm{w}(\mathrm{i}+1))$ $*$ psi $*$ alpha_ $\left.2 * \mathrm{xi}_{-} \mathrm{w} /\left(\mathrm{alph}_{-} 1 * \mathrm{xi} \mathrm{i}_{-} \mathrm{d}\right)\right) *(\mathrm{D}(\mathrm{i})+\mathrm{h} 2 * \mathrm{~m} 34)-$ $\mathrm{xi}-\mathrm{W} *(\mathrm{~W}(\mathrm{i})+\mathrm{h} 2 * \mathrm{~m} 33) ;$ $\mathrm{m} 44=\mathrm{xi}{ }_{-} \mathrm{d} *(1-\mathrm{r} 2-\mathrm{v}(\mathrm{i}+1)) *(\mathrm{I}(\mathrm{i})+\mathrm{h} 2 * \mathrm{~m} 32)-(\mathrm{xi}-\mathrm{d} *(\mathrm{D}(\mathrm{i})+\mathrm{h} 2$ $* \mathrm{~m} 34))$; $\mathrm{S}(\mathrm{i}+1)=\mathrm{S}(\mathrm{i})+(\mathrm{h} / 6) *(\mathrm{~m} 11+2 * \mathrm{~m} 21+2 * \mathrm{~m} 31+\mathrm{m} 41) ;$ $\mathrm{I}(\mathrm{i}+1)=\mathrm{I}(\mathrm{i})+(\mathrm{h} / 6) *(\mathrm{~m} 12+2 * \mathrm{~m} 22+2 * \mathrm{~m} 32+\mathrm{m} 42) ;$ 


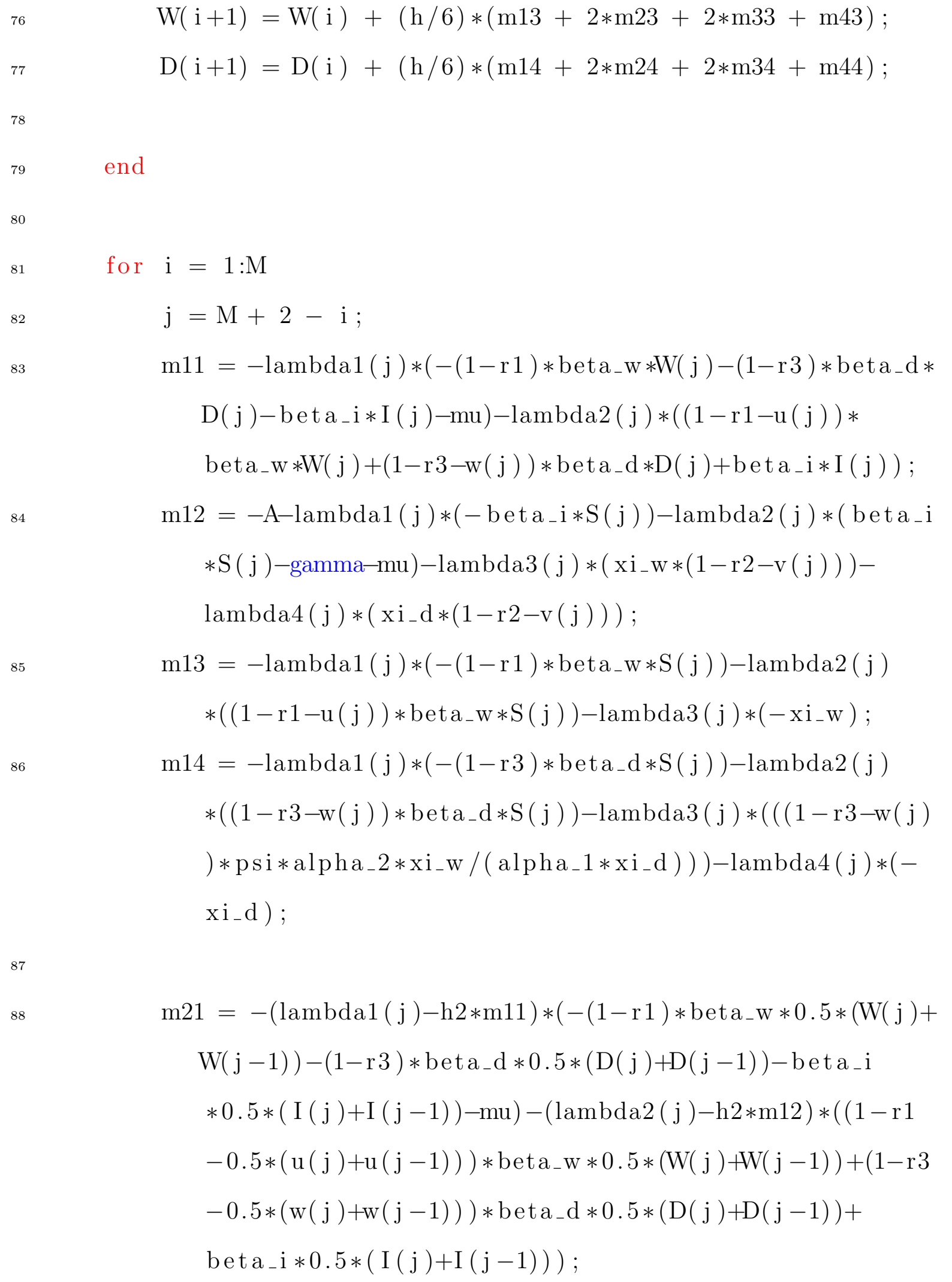




$$
\mathrm{m} 22=-\mathrm{A}-(\operatorname{lambda} 1(\mathrm{j})-\mathrm{h} 2 * \mathrm{~m} 11) *\left(-\mathrm{beta}_{-} \mathrm{i} * 0.5 *(\mathrm{~S}(\mathrm{j})+\mathrm{S}(\mathrm{j}\right.
$$

$-1)))-(\operatorname{lambda} 2(\mathrm{j})-\mathrm{h} 2 * \mathrm{~m} 12) *\left(\operatorname{beta}_{-} \mathrm{i} * 0.5 *(\mathrm{~S}(\mathrm{j})+\mathrm{S}(\mathrm{j}-1)\right.$

)$-\operatorname{gamma}-\mathrm{mu})-(\operatorname{lambda} 3(\mathrm{j})-\mathrm{h} 2 * \mathrm{~m} 13) *(\mathrm{xi}-\mathrm{w} *(1-\mathrm{r} 2-0.5 *(\mathrm{v}$

$(\mathrm{j})+\mathrm{v}(\mathrm{j}-1))))-(\operatorname{lambda} 4(\mathrm{j})-\mathrm{h} 2 * \mathrm{~m} 14) *\left(\mathrm{xi}_{-} \mathrm{d} *(1-\mathrm{r} 2\right.$

$-0.5 *(\mathrm{v}(\mathrm{j})+\mathrm{v}(\mathrm{j}-1))))$;

$\mathrm{m} 23=-(\operatorname{lambda} 1(\mathrm{j})-\mathrm{h} 2 * \mathrm{~m} 11) *\left(-(1-\mathrm{r} 1) * \operatorname{beta}_{-} \mathrm{W} * 0.5 *(\mathrm{~S}(\mathrm{j})+\right.$

$\mathrm{S}(\mathrm{j}-1)))-(\operatorname{lambda} 2(\mathrm{j})-\mathrm{h} 2 * \mathrm{~m} 12) *((1-\mathrm{r} 1-0.5 *(\mathrm{u}(\mathrm{j})+\mathrm{u}($

$\mathrm{j}-1))) *$ beta_ $\left._{-} * 0.5 *(\mathrm{~S}(\mathrm{j})+\mathrm{S}(\mathrm{j}-1))\right)-(\operatorname{lambda} 3(\mathrm{j})-\mathrm{h} 2 *$

$\mathrm{m} 13) *(-\mathrm{xi}-\mathrm{w})$;

$\mathrm{m} 24=-(\operatorname{lambda} 1(\mathrm{j})-\mathrm{h} 2 * \mathrm{~m} 11) *\left(-(1-\mathrm{r} 3) *\right.$ beta_d $_{-} * 0.5 *(\mathrm{~S}(\mathrm{j})+$

$\mathrm{S}(\mathrm{j}-1)))-(\operatorname{lambda} 2(\mathrm{j})-\mathrm{h} 2 * \mathrm{~m} 12) *((1-\mathrm{r} 3-0.5 *(\mathrm{w}(\mathrm{j})+\mathrm{w}(\mathrm{j}$

$-1))) *$ beta $\left._{-} \mathrm{d} * 0.5 *(\mathrm{~S}(\mathrm{j})+\mathrm{S}(\mathrm{j}-1))\right)-(\operatorname{lambda} 3(\mathrm{j})-\mathrm{h} 2 * \mathrm{~m} 13$

)$*\left(\left((1-\mathrm{r} 3-0.5 *(\mathrm{w}(\mathrm{j})+\mathrm{w}(\mathrm{j}-1))) * \mathrm{psi} * \mathrm{alph} \mathrm{a}_{-} 2 * \mathrm{xi} \mathrm{L}_{-} \mathrm{w} /(\right.\right.$

alpha_ $\left.\left.\left.1 * \mathrm{xi}_{-} \mathrm{d}\right)\right)\right)-(\operatorname{lambda} 4(\mathrm{j})-\mathrm{h} 2 * \mathrm{~m} 14) *\left(-\mathrm{xi} \mathrm{d}_{-}\right)$;

$\mathrm{m} 31=-(\operatorname{lambda} 1(\mathrm{j})-\mathrm{h} 2 * \mathrm{~m} 21) *\left(-(1-\mathrm{r} 1) *\right.$ beta $_{-} \mathrm{W} * 0.5 *(\mathrm{~W}(\mathrm{j})+$

$\mathrm{W}(\mathrm{j}-1))-(1-\mathrm{r} 3) * \mathrm{beta}_{-} \mathrm{d} * 0.5 *(\mathrm{D}(\mathrm{j})+\mathrm{D}(\mathrm{j}-1))-\mathrm{beta}_{-} \mathrm{i}$

$* 0.5 *(\mathrm{I}(\mathrm{j})+\mathrm{I}(\mathrm{j}-1))-\mathrm{mu})-(\operatorname{lambda} 2(\mathrm{j})-\mathrm{h} 2 * \mathrm{~m} 22) *((1-\mathrm{r} 1$

$-0.5 *(\mathrm{u}(\mathrm{j})+(\mathrm{u}(\mathrm{j}-1)))) * \operatorname{beta}_{-} \mathrm{w} * 0.5 *(\mathrm{~W}(\mathrm{j})+\mathrm{W}(\mathrm{j}-1))+(1-$

$\mathrm{r} 3-0.5 *(\mathrm{w}(\mathrm{j})+\mathrm{w}(\mathrm{j}-1))) *$ beta $_{-} \mathrm{d} * 0.5 *(\mathrm{D}(\mathrm{j})+\mathrm{D}(\mathrm{j}-1))+$

bet $\left.a_{-} \mathrm{i} * 0.5 *(\mathrm{I}(\mathrm{j})+\mathrm{I}(\mathrm{j}-1))\right)$;

$\mathrm{m} 32=-\mathrm{A}-\left(\operatorname{lambda}_{2}(\mathrm{j})-\mathrm{h} 2 * \mathrm{~m} 21\right) *\left(-\mathrm{beta}_{-} \mathrm{i} * 0.5 *(\mathrm{~S}(\mathrm{j})+\mathrm{S}(\mathrm{j}\right.$

$-1)))-(\operatorname{lambda} 2(\mathrm{j})-\mathrm{h} 2 * \mathrm{~m} 22) *\left(\right.$ bet a $_{-} \mathrm{i} * 0.5 *(\mathrm{~S}(\mathrm{j})+\mathrm{S}(\mathrm{j}-1)$

)$-\operatorname{gamma}-\mathrm{mu})-(\operatorname{lambda} 3(\mathrm{j})-\mathrm{h} 2 * \mathrm{~m} 23) *(\mathrm{xi}-\mathrm{W} *(1-\mathrm{r} 2-0.5 *(\mathrm{v}$

$(\mathrm{j})+\mathrm{v}(\mathrm{j}-1))))-(\operatorname{lambda} 4(\mathrm{j})-\mathrm{h} 2 * \mathrm{~m} 24) *\left(\mathrm{xi} \mathrm{d}_{-} \mathrm{d} *(1-\mathrm{r} 2\right.$

$-0.5 *(\mathrm{v}(\mathrm{j})+\mathrm{v}(\mathrm{j}-1))))$; 
$\mathrm{m} 33=-(\operatorname{lambda} 1(\mathrm{j})-\mathrm{h} 2 * \mathrm{~m} 21) *\left(-(1-\mathrm{r} 1) * \operatorname{beta}_{-} \mathrm{w} * 0.5 *(\mathrm{~S}(\mathrm{j})+\right.$ $\mathrm{S}(\mathrm{j}-1)))-(\operatorname{lambda} 2(\mathrm{j})-\mathrm{h} 2 * \mathrm{~m} 22) *((1-\mathrm{r} 1-0.5 *(\mathrm{u}(\mathrm{j})+\mathrm{u}($ $\mathrm{j}-1))) *$ beta_w $* 0.5 *(\mathrm{~S}(\mathrm{j})+\mathrm{S}(\mathrm{j}-1)))-(\operatorname{lambda} 3(\mathrm{j})-\mathrm{h} 2 *$ $\mathrm{m} 23) *(-\mathrm{xi}-\mathrm{w})$;

$\mathrm{m} 34=-(\operatorname{lambda} 1(\mathrm{j})-\mathrm{h} 2 * \mathrm{~m} 21) *\left(-(1-\mathrm{r} 3) *\right.$ beta_d $_{-} * 0.5 *(\mathrm{~S}(\mathrm{j})+$ $\mathrm{S}(\mathrm{j}-1)))-(\operatorname{lambda} 2(\mathrm{j})-\mathrm{h} 2 * \mathrm{~m} 22) *((1-\mathrm{r} 3-0.5 *(\mathrm{w}(\mathrm{j})+\mathrm{w}(\mathrm{j}$ $-1))) *$ beta_d $* 0.5 *(\mathrm{~S}(\mathrm{j})+\mathrm{S}(\mathrm{j}-1)))-(\operatorname{lambda} 3(\mathrm{j})-\mathrm{h} 2 * \mathrm{~m} 23$ )$*\left(\left((1-\mathrm{r} 3-0.5 *(\mathrm{w}(\mathrm{j})+\mathrm{w}(\mathrm{j}-1))) * \mathrm{psi} * \mathrm{alph} \mathrm{a}_{-} 2 * \mathrm{xi} \_\mathrm{w} /(\right.\right.$ alpha_1*xi_d ) ) $)-(\operatorname{lambda} 4(\mathrm{j})-\mathrm{h} 2 * \operatorname{m} 24) *\left(-\mathrm{xi} \mathrm{d}_{-} \mathrm{d}\right)$;

$\mathrm{m} 41=-(\operatorname{lambda1}(\mathrm{j})-\mathrm{h} 2 * \mathrm{~m} 31) *\left(-(1-\mathrm{r} 1) *\right.$ beta $_{-} \mathrm{W} * \mathrm{~W}(\mathrm{j}-1)-(1-$ r 3$) *$ beta_d $* D(j-1)-$ beta_i $i * I(j-1)-m u)-(\operatorname{lambda} 2(j)-h 2$ $* \mathrm{~m} 32) *\left((1-\mathrm{r} 1-(\mathrm{u}(\mathrm{j}-1))) *\right.$ beta $_{-} \mathrm{W} * \mathrm{~W}(\mathrm{j}-1)+(1-\mathrm{r} 3-\mathrm{w}(\mathrm{j}-1))$ *beta ${ }_{-} d * D(j-1)+$ bet $\left._{-}{ }_{-} i * I(j-1)\right)$; $\mathrm{m} 42=-\mathrm{A}-(\operatorname{lambda} 1(\mathrm{j})-\mathrm{h} 2 * \mathrm{~m} 31) *\left(-\mathrm{beta}_{-} \mathrm{i} * \mathrm{~S}(\mathrm{j}-1)\right)-($

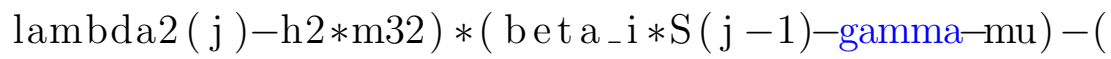
$\operatorname{lambda} 3(\mathrm{j})-\mathrm{h} 2 * \mathrm{~m} 33) *\left(\mathrm{xi} \_\mathrm{w} *(1-\mathrm{r} 2-\mathrm{v}(\mathrm{j}-1))\right)-(\operatorname{lambda} 4(\mathrm{j}$ )$-\mathrm{h} 2 * \mathrm{~m} 34) *(\mathrm{xi}-\mathrm{d} *(1-\mathrm{r} 2-\mathrm{v}(\mathrm{j}-1)))$; $\mathrm{m} 43=-(\operatorname{lambda} 1(\mathrm{j})-\mathrm{h} 2 * \mathrm{~m} 31) *\left(-(1-\mathrm{r} 1) * \operatorname{beta}_{-} \mathrm{W} * \mathrm{~S}(\mathrm{j}-1)\right)-($ $\operatorname{lambda} 2(\mathrm{j})-\mathrm{h} 2 * \mathrm{~m} 32) *\left((1-\mathrm{r} 1-\mathrm{u}(\mathrm{j}-1)) * \operatorname{beta}_{-} \mathrm{W} * \mathrm{~S}(\mathrm{j}-1)\right)-($ $\operatorname{lambda} 3(\mathrm{j})-\mathrm{h} 2 * \mathrm{~m} 33) *\left(-\mathrm{xi} \mathrm{i}_{-} \mathrm{w}\right)$; $\mathrm{m} 44=-(\operatorname{lambda} 1(\mathrm{j})-\mathrm{h} 2 * \mathrm{~m} 31) *\left(-(1-\mathrm{r} 3) * \mathrm{beta}_{-} \mathrm{d} * \mathrm{~S}(\mathrm{j}-1)\right)-($ $\operatorname{lambda} 2(\mathrm{j})-\mathrm{h} 2 * \mathrm{~m} 32) *\left((1-\mathrm{r} 3-\mathrm{w}(\mathrm{j}-1)) * \mathrm{beta}_{-} \mathrm{d} * \mathrm{~S}(\mathrm{j}-1)\right)-($ $\operatorname{lambda} 3(\mathrm{j})-\mathrm{h} 2 * \mathrm{~m} 33) *\left(\left((1-\mathrm{r} 3-\mathrm{w}(\mathrm{j}-1)) * \mathrm{psi} * \operatorname{alph}_{-} 2 *\right.\right.$ xi_w / ( alpha_1*xi_d $)))-(\operatorname{lambda} 4(\mathrm{j})-\mathrm{h} 2 * \operatorname{m} 34) *\left(-\mathrm{xi} \mathrm{i}_{-} \mathrm{d}\right)$; 


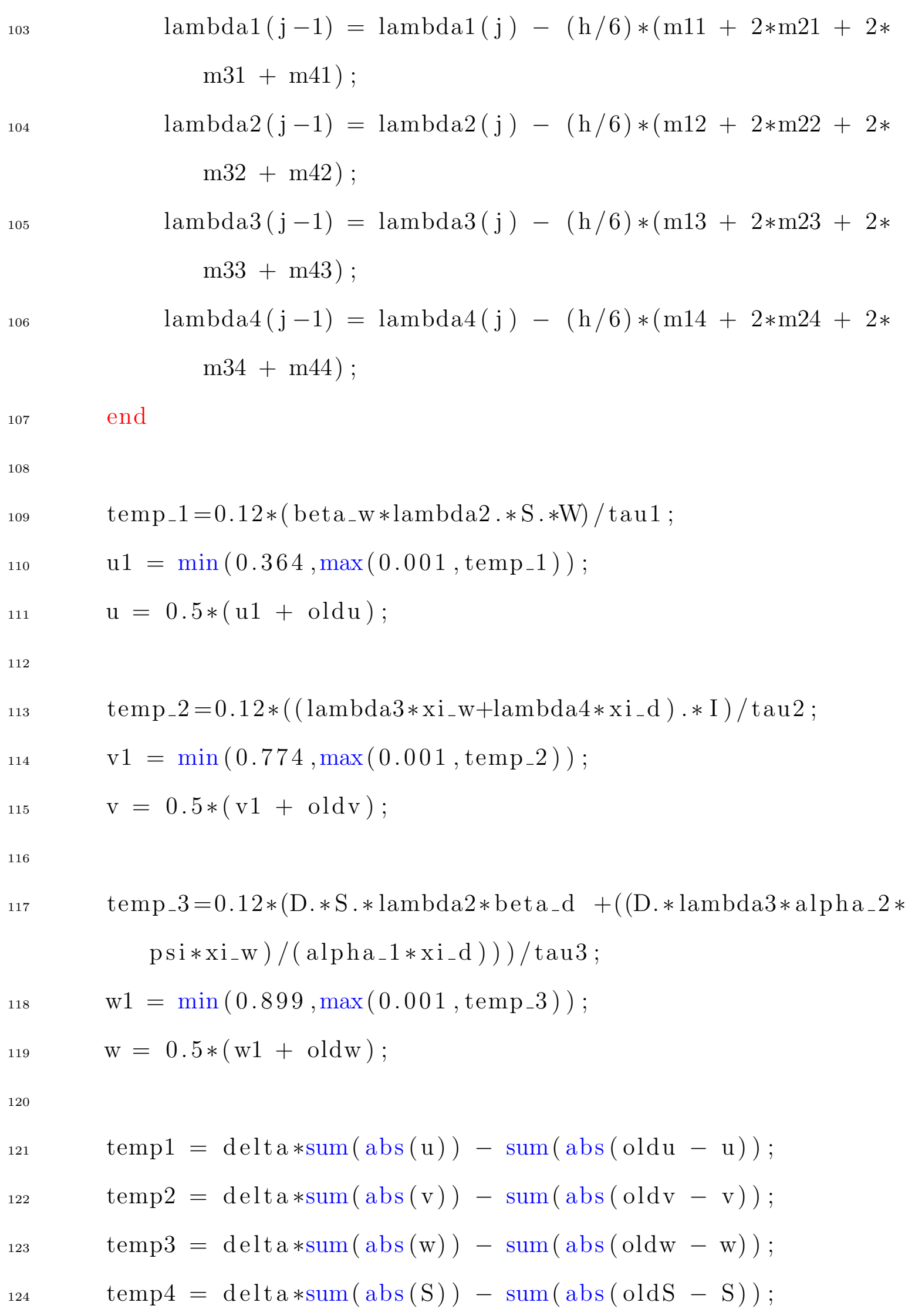

$\operatorname{lambda1}(\mathrm{j}-1)=\operatorname{lambda1}(\mathrm{j})-(\mathrm{h} / 6) *(\mathrm{~m} 11+2 * \mathrm{~m} 21+2 *$ $\mathrm{m} 31+\mathrm{m} 41)$

$\operatorname{lambda} 2(\mathrm{j}-1)=\operatorname{lambda} 2(\mathrm{j})-(\mathrm{h} / 6) *(\mathrm{~m} 12+2 * \mathrm{~m} 22+2 *$ $\mathrm{m} 32+\mathrm{m} 42)$

$\operatorname{lambda} 3(\mathrm{j}-1)=\operatorname{lambda} 3(\mathrm{j})-(\mathrm{h} / 6) *(\mathrm{~m} 13+2 * \mathrm{~m} 23+2 *$ $\mathrm{m} 33+\mathrm{m} 43)$

$\operatorname{lambda} 4(\mathrm{j}-1)=\operatorname{lambda} 4(\mathrm{j})-(\mathrm{h} / 6) *(\mathrm{~m} 14+2 * \mathrm{~m} 24+2 *$ $\mathrm{m} 34+\mathrm{m} 44)$

end

temp_1 $=0.12 *($ beta_w $*$ lambda $2 . * \mathrm{~S} . * \mathrm{~W}) /$ tau 1

$\mathrm{u} 1=\min \left(0.364, \max \left(0.001\right.\right.$, temp_1 $\left.\left._{-}\right)\right)$;

$\mathrm{u}=0.5 *(\mathrm{u} 1+\mathrm{old} u)$

temp_2 $=0.12 *\left(\left(\operatorname{lambda} 3 * \mathrm{xi}_{-} \mathrm{w}+\mathrm{lambda} 4 * \mathrm{x} \mathrm{i}_{-} \mathrm{d}\right) . * \mathrm{I}\right) / \operatorname{tau} 2$;

$\mathrm{v} 1=\min \left(0.774, \max \left(0.001\right.\right.$, temp_2 $\left.\left._{-}\right)\right)$;

$\mathrm{v}=0.5 *(\mathrm{v} 1+\mathrm{old} \mathrm{v})$

temp_3 $=0.12 *($ D. $*$ S.$*$ lambda $2 *$ beta_d $+((D . * \operatorname{lambda} 3 *$ alpha_2 $*$ psi $\left.* \mathrm{xi}_{-} \mathrm{w}\right) /\left(\right.$ alpha_$\left.\left.\left._{-} 1 * \mathrm{xi}_{-} \mathrm{d}\right)\right)\right) / \operatorname{tau} 3$;

$\mathrm{w} 1=\min \left(0.899, \max \left(0.001\right.\right.$, temp_3 $\left.\left._{-}\right)\right)$;

$\mathrm{w}=0.5 *(\mathrm{w} 1+\mathrm{old} \mathrm{w}) ;$

$\operatorname{temp} 1=\operatorname{delta} * \operatorname{sum}(\operatorname{abs}(u))-\operatorname{sum}(\operatorname{abs}(\operatorname{old} u-u))$

temp2 $=\operatorname{delta} * \operatorname{sum}(\operatorname{abs}(\mathrm{v}))-\operatorname{sum}(\operatorname{abs}(\operatorname{old} v-\mathrm{v})) ;$

temp3 $=\operatorname{delta} * \operatorname{sum}(\operatorname{abs}(\mathrm{w}))-\operatorname{sum}(\operatorname{abs}(\operatorname{old} w-\mathrm{w})) ;$

temp4 $=\operatorname{delta} * \operatorname{sum}(\operatorname{abs}(S))-\operatorname{sum}(\operatorname{abs}(\operatorname{old} S-S)) ;$ 


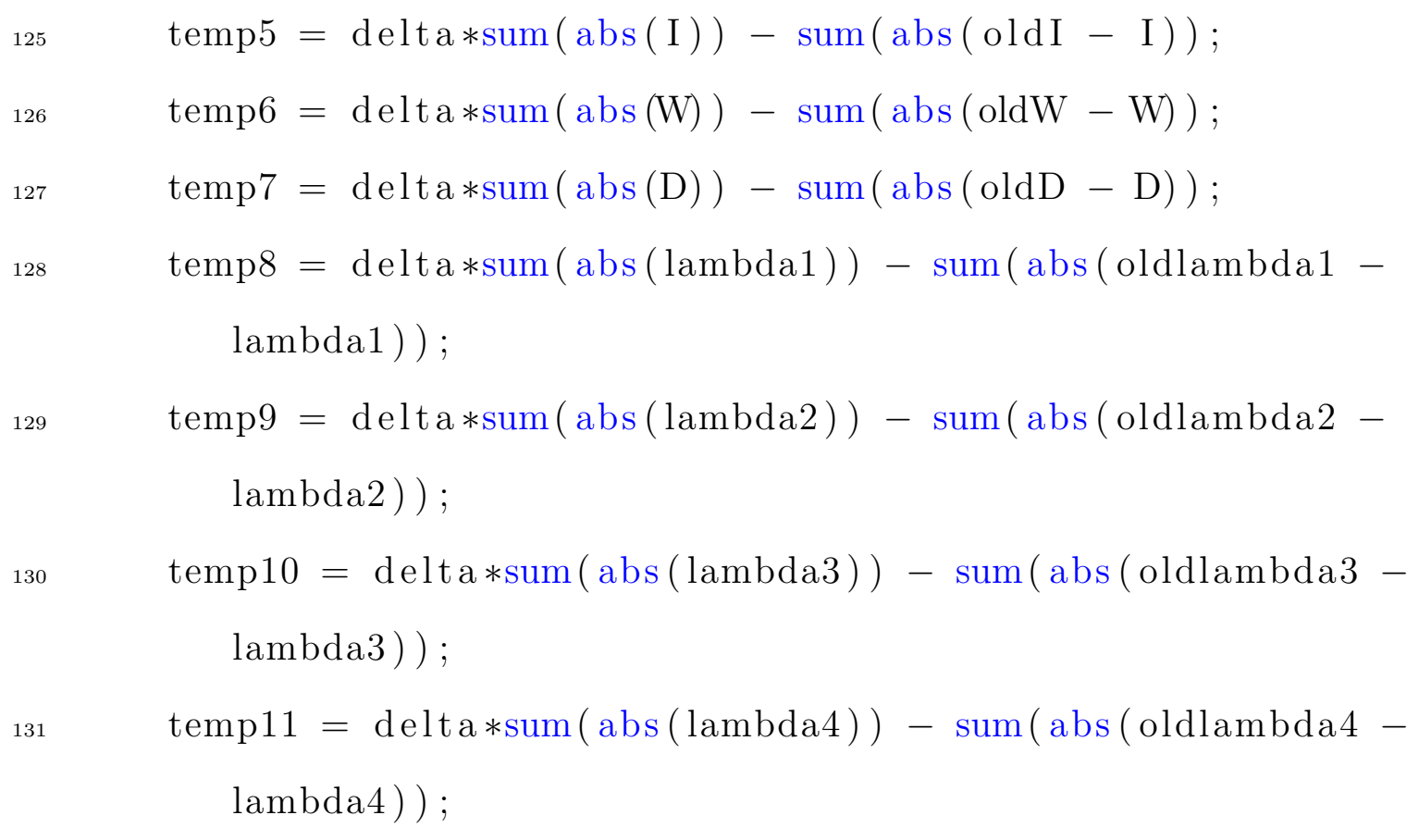

132 test $=\min ($ temp $1, \min ($ temp2, $\min ($ temp3, $\min ($ temp $4, \min ($ temp5, $\min (t e m p 6, \min (t e m p 7, \min (t e m p 8, \min (t e m p 9, \min$

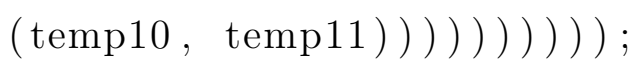

end

\section{for $\quad \mathrm{i}=1: \mathrm{M}$}

$\mathrm{m} 1=\operatorname{gamma} * \mathrm{I}(\mathrm{i})-\mathrm{mu} * \mathrm{R}(\mathrm{i})+\mathrm{u}(\mathrm{i}) * \operatorname{beta}_{-} \mathrm{W} * \mathrm{~S}(\mathrm{i}) * \mathrm{~W}(\mathrm{i})+\mathrm{w}(\mathrm{i}) *$ beta $\mathrm{d}_{-} * \mathrm{~S}(\mathrm{i}) * \mathrm{D}(\mathrm{i})$;

$\mathrm{m} 2=\operatorname{gamma} * 0.5 *(\mathrm{I}(\mathrm{i})+\mathrm{I}(\mathrm{i}+1))-\mathrm{mu} *(\mathrm{R}(\mathrm{i})+\mathrm{h} 2 * \mathrm{~m} 1)+0.5 *(\mathrm{u}(\mathrm{i})+\mathrm{u}$ $(\mathrm{i}+1)) *$ beta $_{-} \mathrm{W} * 0.5 *(\mathrm{~S}(\mathrm{i})+\mathrm{S}(\mathrm{i}+1)) * 0.5 *(\mathrm{~W}(\mathrm{i})+\mathrm{W}(\mathrm{i}+1))$ $+0.5 *(\mathrm{w}(\mathrm{i})+\mathrm{w}(\mathrm{i}+1)) * \mathrm{beta}_{-} \mathrm{d} * 0.5 *(\mathrm{~S}(\mathrm{i})+\mathrm{S}(\mathrm{i}+1)) * 0.5 *(\mathrm{D}(\mathrm{i})+$ $\mathrm{D}(\mathrm{i}+1))$;

$\mathrm{m} 3=\operatorname{gamma} * 0.5 *(\mathrm{I}(\mathrm{i})+\mathrm{I}(\mathrm{i}+1))-\mathrm{mu} *(\mathrm{R}(\mathrm{i})+\mathrm{h} 2 * \mathrm{~m} 2)+0.5 *(\mathrm{u}(\mathrm{i})+\mathrm{u}$ $(\mathrm{i}+1)) *$ beta $_{-} \mathrm{W} * 0.5 *(\mathrm{~S}(\mathrm{i})+\mathrm{S}(\mathrm{i}+1)) * 0.5 *(\mathrm{~W}(\mathrm{i})+\mathrm{W}(\mathrm{i}+1))$ 


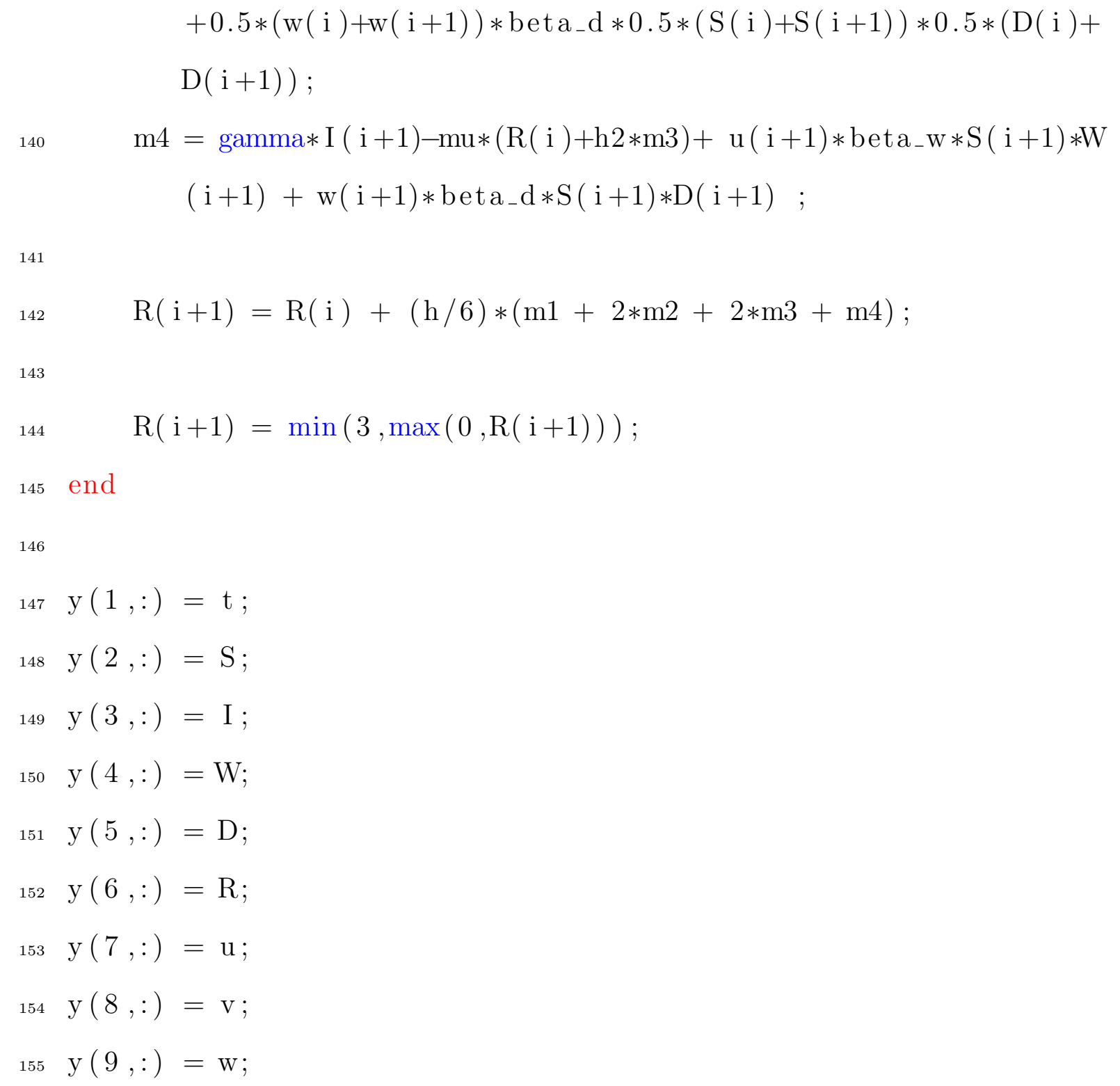




\section{Bibliography}

[1] S. Abul, "Environmental and health impact of solid waste disposal at Mangwaneni dumpsite in Manzini: Swaziland," Journal of Sustainable Development in Africa, vol. 12 , no. 7,2010 , pp. 64-78.

[2] J. Bartram, S. Cairncross, "Hygiene, sanitation, and water: forgotten foundations of health," PLoS Med, vol. 7, no. 11, 2010, pp. e1000367.

[3] E. J. Barzilay, N. Schaad,R. Magloire, K. S. Mung, J. Boncy, G. A. Dahourou, E. D. Mintz, M. W. Steenland, J. F. Vertefeuille, J. W. Tappero, "Cholera surveillance during the Haiti epidemic-the first 2 years," New England Journal of Medicine, vol. 368, no. 7, 2013, pp. 599-609.

[4] N. G. Becker, D. N. Starczak, "Optimal vaccination strategies for a community of households," Mathematical Biosciences, vol. 139, no. 2, 1997, pp. 117-132.

[5] H. Behncke, "Optimal control of deterministic epidemics," Optimal Control Applications and Methods, vol. 21, no. 6, 2000, pp. 269-285.

[6] A. M. Bouabid, "A systems approach for the selection of appropriate water supply and sanitation infrastructure in developing communities," PhD Thesis, University of Virginia, 2013.

[7] A. M. Bouabid, "Requirements analysis for sustainable sanitation systems in low-income countries," Master's Thesis, University of Virginia, 2004. 
[8] A. Bras, C. Berdier, E. Emmanuel, M. Zimmerman, "Problems and current practices of solid waste management in Port-au-Prince (Haiti)," Waste Management, vol. 29, no. 11, 2009, pp. 2907-2909.

[9] S. Cairncross, V. Valdmanis, "Water supply, sanitation, and hygiene promotion," in Disease Control Priorities in Developing Countries, World Bank, Washington (DC), 2006.

[10] G. Caldarelli, Scale-free networks: complex webs in nature and technology, Oxford University Press, 2007.

[11] J. Carr, Applications of centre manifold theory, Springer Science \& Business Media, 2012.

[12] C. Castilho, "Optimal control of an epidemic through educational campaigns," Electronic Journal of Differential Equations, vol. 2006, no. 125, 2000, pp. 1-11.

[13] C. Castillo-Chávez, Z. Feng, W. Huang, "On the computation of $R_{0}$ and its role on global stability," Mathematical Approaches for Emerging and Reemerging Infectious Diseases: an Introduction, vol. 1, 2002, pp. 229.

[14] C. Castillo-Chavez, B. Song, "Dynamical models of tuberculosis and their applications," Mathematical Biosciences and Engineering, vol. 1, no. 2, 2004, pp. $361-404$.

[15] P. Chalmin, C. Gaillochet, "From waste to resource: an abstract of the world waste survey 2009," Technical Report, Available: http://www.rebnews.com/pdfs/ news/from_waste_to_resource_report_veolia.pdf, 2009.

[16] CIA Factbook, "Haiti ," Technical Report, Available: https://www.cia.gov/ library/publications/the-world-factbook/geos/ha.html, 2016. 
[17] CIA Factbook, "Country comparison :: birth rate," Technical Report, Available: https://www.cia.gov/library/publications/the-world-factbook/rankorder/ 2054rank.html, 2016.

[18] CIA Factbook, "Country comparison :: death rate ," Technical Report, Available: https://www.cia.gov/library/publications/the-world-factbook/rankorder/ 2066rank.html, 2016.

[19] D. Clancy, A. B. Piunovskiy, "An explicit optimal isolation policy for a deterministic epidemic model," Applied Mathematics and Computation, vol. 163, no. 3, 2005, pp. 1109-1121.

[20] B. J. Coburn, B. G. Wagner, S. Blower, "Modeling influenza epidemics and pandemics: insights into the future of swine flu (H1N1)," BMC Medicine, vol. 7, no. 1, 2009, pp. 30 .

[21] S. Cointreau, "Occupational and environmental health issues of solid waste management: special emphasis on middle-and lower-income countries," Technical Report, Available: http://siteresources.worldbank.org/INTUSWM/Resources/ up-2.pdf, 2006.

[22] M. H. A. Davis, M. A. H. Dempster, S. P. Sethi, D. Vermes, "Optimal capacity expansion under uncertainty," Advances in Applied Probability, 1987, pp. 156-176.

[23] M. C. Eisenberg, S. L. Robertson, J. H. Tien, "Identifiability and estimation of multiple transmission pathways in cholera and waterborne disease," Journal of Theoretical Biology, vol. 324, 2013, pp. 84-102.

[24] S. A. Esrey, R. G. Feachem, J. M. Hughes, "Interventions for the control of diarrhoeal diseases among young children: improving water supplies and excreta disposal facilities," Bulletin of the World Health Organization, vol. 63, no. 4, 1985, pp. 757. 
[25] S. A. Esrey, J. B. Potash, L. Roberts, C. Shiff, "Effects of improved water supply and sanitation on ascariasis, diarrhoea, dracunculiasis, hookworm infection, schistosomiasis, and trachoma," Bulletin of the World Health Organization, vol. 69, no. 5, 1991, pp. 609.

[26] L. Fewtrell, R. B. Kaufmann, D. Kay, W. Enanoria, L. Haller, J. M. Colford, "Water, sanitation, and hygiene interventions to reduce diarrhoea in less developed countries: a systematic review and meta-analysis," The Lancet Infectious Diseases, vol. 5, no. 1, 2005, pp. 42-52.

[27] I. C. H. Fung, "Cholera transmission dynamic models for public health practitioners," Emerging Themes in Epidemiology, vol. 11, 2014, pp. 1.

[28] C. P. Gerba, "Microbial pathogens in municipal solid waste," in Microbiology of Solid Waste, 1996, pp. 155-173.

[29] L. Giusti, "A review of waste management practices and their impact on human health," Waste Management, vol. 29, no. 8, 2009, pp. 2227-2239.

[30] C. Godsil, G. Royle, Algebraic Graph Theory, Springer Science \& Business Media, 2001 .

[31] T. K. Graczyk, M. Kacprzak, E. Neczaj, L. Tamang, H. Graczyk, F. E. Lucy, A. S. Girouard, "Occurrence of cryptosporidium and giardia in sewage sludge and solid waste landfill leachate and quantitative comparative analysis of sanitization treatments on pathogen inactivation," Environmental Research, vol. 106, no. 1, 2008, pp. 27-33.

[32] E. Grisey, E. Belle, J. Dat, J. Mudry, L. Aleya, "Survival of pathogenic and indicator organisms in groundwater and landfill leachate through coupling bacterial enumeration with tracer tests," Desalination, vol. 261, no. 1, 2010, pp. 162-168. 
[33] P. Harremoës, "Integrated water and waste management," Water Science and Technology, vol. 35, no. 9, 1997, pp. 11-20.

[34] T. R. Hendrix, "The pathophysiology of cholera," Bulletin of the New York Academy of Medicine, vol. 47, no. 10, 1971, pp. 1169.

[35] G. Hutton, L. Haller, "Evaluation of the costs and benefits of water and sanitation improvements at the global level," Technical Report, Available: http://www.who. int/water_sanitation_health/wsh0404.pdf, 2004.

[36] D. Iacoviello, N. Stasio, "Optimal control for SIRC epidemic outbreak," Computer Methods and Programs in Biomedicine, vol. 110, no. 3, 2013, pp. 333-342.

[37] W. O. Kermack, A. G. McKendrick, "A contribution to the mathematical theory of epidemics," Proceedings of the Royal Society of London A: Mathematical, Physical and Engineering Sciences, vol. 115, no. 772, 1927, pp. 700-721.

[38] G. T. Keusch, O. Fontaine, A. Bhargava, C. Boschi-Pinto, Z. A. Bhutta, E. Gotuzzo, J. Rivera, J. Chow, S. A. Shahid-Salles, R. Laxminarayan, "Diarrheal diseases," Disease Control Priorities in Developing Countries, vol. 2, 2006, pp. $371-388$.

[39] A. A. King, E. L. Ionides, M. Pascual, M. J. Bouma, "Inapparent infections and cholera dynamics," Nature, vol. 454, no. 7206, 2008, pp. 877-880.

[40] P. Kjeldsen, "Groundwater pollution source characterization of an old landfill," Journal of Hydrology, vol. 142, no. 1, 1993, pp. 349-371.

[41] S. Lenhart, J. T. Workman, Optimal control applied to biological models, CRC Press, 2007.

[42] M. K. Lo-Karija, Q. I. Shihua, Y. Y. Ziggah, "Correlation among municipal solid waste pollution, fecal coliform water pollution and water borne diseases in 
Juba/South Sudan," British Journal of Applied Science \& Technology, vol. 3, no. 4, 2013.

[43] G. E. Louis, L. M. Magpili, "A life-cycle capacity-based approach to allocating investments in municipal sanitation infrastructure," Structure and Infrastructure Engineering, vol. 3, no. 2, 2007, pp. 121-131.

[44] G. E. Louis, L. M. Magpili, J. Rogers, J. Henriques, "Integrated municipal sanitation systems for efficient management of public water and sanitation services," Proceedings of International Annual Conference of the American Society of Engineering Management, Minneapolis, MN, 2013.

[45] H. Luss, "Operations research and capacity expansion problems: a survey," Operations Research, vol. 30, no. 5, 1982, pp. 907-947.

[46] L. M. Magpili, "An impact-based method for the capacity planning of sanitation services in lower income countries," PhD Thesis, University of Virginia, 2003.

[47] D. Mara, J. Lane, B. Scott, D. Trouba, "Sanitation and health," PLoS Medicine, vol. 7, no. 11, 2010, pp. 1359.

[48] X. Meng, L. Chen, "The dynamics of a new SIR epidemic model concerning pulse vaccination strategy," Applied Mathematics and Computation, vol. 197, no. 2, 2008, pp. 582-597.

[49] P. Modak, Y. Jiemian, Y. Hongyuan, C. R. Mohanty, "Shanghai manual-a guide for sustainable urban development in the 21st century," United Nations. Vol. 2012, 2012.

[50] M. A. Montgomery, M. Elimelech, "Water and sanitation in developing countries: including health in the equation," Environmental Science \& Technology, vol. 41, no. 1, 2007, pp. 17-24. 
[51] J. G. Morris Jr, "Cholera-modern pandemic disease of ancient lineage," Emerg Infect Dis, vol. 17, no. 11, 2011, pp. 2099-2104.

[52] Z. Mukandavire, D. L. Smith, J. G. Morris Jr, "Cholera in Haiti: reproductive numbers and vaccination coverage estimates," Scientific Reports, vol. 3, 2013.

[53] K. L. Nichol, K. Tummers, A. Hoyer-Leitzel, J. Marsh, M. Moynihan, S. McKelvey, "Modeling seasonal influenza outbreak in a closed college campus: impact of preseason vaccination, in-season vaccination and holidays/breaks," PloS One, vol. 5, no. 3, 2010, pp. e9548.

[54] L. P. Onn, "Water management issues in Singapore," Water in Mainland Southeast Asia, vol. 29, 2005.

[55] Pan American Health Organization, "Atlas of cholera outbreak in la Hispaniola, 2010-2015," Technical Report, Available: http://new.paho.org/hq/images/Atlas_ IHR/CholeraHispaniola/atlas.html, 2015.

[56] Polk County, "Polk county water, sewage, and solid waste authority," Technical Report, Available: http://www.polkwaterauthority.com, 2015.

[57] L. S. Pontryagin, Mathematical theory of optimal processes, CRC Press, 1987.

[58] A. Prüss, D. Kay, L. Fewtrell, J. Bartram, "Estimating the burden of disease from water, sanitation, and hygiene at a global level," Environmental Health Perspectives, vol. 110, no. 5, 2002, pp. 537-542.

[59] M. H. Romdhana, D. Lecomte, B. Ladevie, C. Sablayrolles, "Monitoring of pathogenic microorganisms contamination during heat drying process of sewage sludge," Process Safety and Environmental Protection, vol. 87, no. 6, 2009, pp. 377-386. 
[60] J. A. Salvato, Environmental Engineering and Sanitation, John Wiley \& Sons, 1992.

[61] J. A. Salvato, N. L. Nemerow, F. J. Agardy, Environmental engineering, John Wiley \& Sons, New York, NY, 2003.

[62] F. P. Sankoh, X. Yan, Q. Tran , "Environmental and health impact of solid waste disposal in developing cities: a case study of Granville Brook dumpsite, Freetown, Sierra Leone," Journal of Environmental Protection, 2013.

[63] K. E. Schilling, E. Porter, Urban water infrastructure. Vol. 180. Springer Science \& Business Media, 2012.

[64] Seattle Public Utilities (SPU), "Seattle public utilities," Technical Report, Available: http://www.seattle.gov/util/AboutUs/index.htm, 2015.

[65] O. Sharomi, A. B. Gumel, "Curtailing smoking dynamics: a mathematical modeling approach," Applied Mathematics and Computation, vol. 195, no. 2, 2008, pp. 475-499.

[66] M. Strauss, A. Montangero, "Feacal sludge management review of practices, problems and initiatives," Technical Report, Available: http://r4d.dfid.gov.uk/ PDF/Outputs/Water/R8056-FS.pdf, 2002.

[67] Supply, Water and Council, Sanitation Collaborative and UNICEF, "Global water supply and sanitation assessment 2000 report," Technical Report, Available: http://www.who.int/water_sanitation_health/monitoring/jmp2000.pdf, 2000.

[68] The DHS Program Demographic and Health Surveys, "Data," Technical Report, Available: http://dhsprogram.com/data/, 2015. 
[69] J. H. Tien, D. J. Earn, "Multiple transmission pathways and disease dynamics in a waterborne pathogen model," Bulletin of Mathematical Biology, vol. 72, no. 6, 2010, pp. 1506-1533.

[70] A. R. Tuite, J. Tien, M. Eisenberg, D. J. Earn, J. Ma, D. N. Fisman, "Cholera epidemic in Haiti, 2010: using a transmission model to explain spatial spread of disease and identify optimal control interventions," Annals of Internal Medicine, vol. 154, no. 9, 2011, pp. 593-601.

[71] U.S. Environmental Protection Agency, "Solid waste: RCRA subtitle D," Technical Report, Available: http://www.epa.gov/region2/waste/dsummary.htm, 2015.

[72] UNICEF, "Cholera toolkit," Technical Report, Available: http://www.unicef.org/ cholera/Cholera-Toolkit-2013.pdf, 2013.

[73] United Nations, "World population prospects: the 2012 revision," Technical Report, Available: http://esa.un.org/wpp/, 2013.

[74] P. Van den Driessche and James Watmough, "Further notes on the basic reproduction number," Mathematical Epidemiology, 2008, pp. 159-178.

[75] P. Van den Driessche, J. Watmough, "Reproduction numbers and sub-threshold endemic equilibria for compartmental models of disease transmission," Mathematical Biosciences, vol. 180, no. 1, 2002, pp. 29-48.

[76] J. A. Van Mieghem, "Commissioned paper: capacity management, investment, and hedging: review and recent developments," Manufacturing \& Service Operations Management, vol. 5, no. 4, 2003, pp. 269-302.

[77] E. Verriest, F. Delmotte, M. Egerstedt, "Control of epidemics by vaccination," Proceedings of the American Control Conference, vol. 2, 2005, pp. 985. 
[78] X. S. Wang, L. Zhong, "Ebola outbreak in West Africa: real-time estimation and multiple-wave prediction," arXiv preprint arXiv:1503.06908, 2015.

[79] World Bank, "Birth rate," Technical Report, Available: http://data.worldbank. org/indicator/SP.DYN.CBRT.IN, 2015.

[80] World Bank, "Country and lending groups," Technical Report, Available: http: //data.worldbank.org/about/country-and-lending-groups, 2015.

[81] World Bank, "Death rate," Technical Report, Available: http://data.worldbank. org/indicator/SP.DYN.CDRT.IN/countries, 2015.

[82] World Bank, "Water supply overview 1," Technical Report, Available: http: //www.worldbank.org/en/topic/water/overview, 2015.

[83] World Bank, "Water supply overview 2," Technical Report, Available: http: //www.worldbank.org/depweb/beyond/beyondco/beg_03.pdf, 2015.

[84] World Bank, "Water supply overview," Technical Report, Available: http://www. oecd.org/dac/stats/49839924.pdf, 2015.

[85] World Bank, "Health through safe drinking water and basic sanitation," Technical Report, Available: http://www.who.int/water_sanitation_health/mdg1/en/print. html, 2006.

[86] World Health Organization, "Global health observatory (GHO) data," Technical Report, Available: http://www.who.int/gho/publications/world_health_statistics/ en/, 2015.

[87] World Health Organization, "25 years progress on sanitaion and drinking water ," Technical Report, Available: http://www.unicef.org/publications/files/Progress_ on_Sanitation_and_Drinking_Water_2015_Update_.pdf, 2015. 
[88] World Health Organization, "Progress on drinking-water and sanitation2012 update," Technical Report, Available: http://www.unicef.org/media/files/ JMPreport2012.pdf, 2012.

[89] World Health Organization, "Cholera," Technical Report, Available: http://www. unicef.org/cholera/Chapter_1_intro/01_WHO_Cholera_Fact_sheet_107_July_2012. pdf, 2012.

[90] World Health Organization, "Combating waterborne disease at the household level," World Health Organization, 2007.

[91] S. D. Wu, M. Erkoc, S. Karabuk, "Managing capacity in the high-tech industry: a review of literature," The Engineering Economist, vol. 50, no. 2, 2005, pp. 125-158. 